[Supporting Information]

\title{
Reduction of Ethynylenes to Vinylenes in Macrocyclic $\pi$-Extended Thiophene Skeleton under McMurry Coupling Conditions
}

\author{
Keigo Shirahata, ${ }^{1}$ Masataka Takashika, ${ }^{1}$ Kazunori Hirabayashi, ${ }^{2}$ Masashi Hasegawa, ${ }^{* 3}$ \\ Hiroyuki Otani, ${ }^{* 1}$ Keitaro Yamamoto, ${ }^{4}$ Yutaka Ie, ${ }^{4}$ Toshio Shimizu, ${ }^{2}$ \\ Shinobu Aoyagi, ${ }^{5}$ and Masahiko Iyoda* ${ }^{2}$
}

\begin{abstract}
${ }^{1}$ Graduate School of Environment and Information Sciences, Yokohama National University, Hodogaya-ku, Yokohama, Kanagawa 240-850, Japan

${ }^{2}$ Department of Chemistry, Graduate School of Science and Engineering, Tokyo Metropolitan University, Hachioji, Tokyo 192-0397, Japan

${ }^{3}$ School of Science, Kitasato University, Sagamihara, Kanagawa 252-0373, Japan

${ }^{4}$ The Institute of Scientific and Industrial Research, Osaka University, Ibaraki, Osaka 567-0047, Japan

${ }^{5}$ Department of Information and Basic Science, Nagoya City University, Nagoya 467-8501, Japan
\end{abstract}

\section{Contents}

S1. ${ }^{1} \mathrm{H}$ and ${ }^{13} \mathrm{C}$ NMR spectra

S2. X-ray crystallographic analysis of $\mathbf{2}, \mathbf{3}$, and $\mathbf{4}$

S3. Geometrical optimization of $\mathbf{2}, \mathbf{3}$, and $\mathbf{4}$

S4. Comparison of total energy and HOMO-LUMO energy level

S5. PXRD patterns of films of $\mathbf{2}, \mathbf{3}$, and $\mathbf{4}$

S6. OFET behavior of $\mathbf{2}, \mathbf{3}$, and 4

S7. Possible intermediates for the McMurry reaction of 1 (theoretical studies) 


\section{S1. ${ }^{1} \mathrm{H}$ and ${ }^{13} \mathrm{C}$ NMR spectra}

Figure S1. ${ }^{1} \mathrm{H}$ and ${ }^{13} \mathrm{C}$ NMR spectra of 2.

(a) ${ }^{1} \mathrm{H}$ NMR spectrum of $2\left(500 \mathrm{MHz}, \mathrm{CDCl}_{3}, 25^{\circ} \mathrm{C}\right)$.
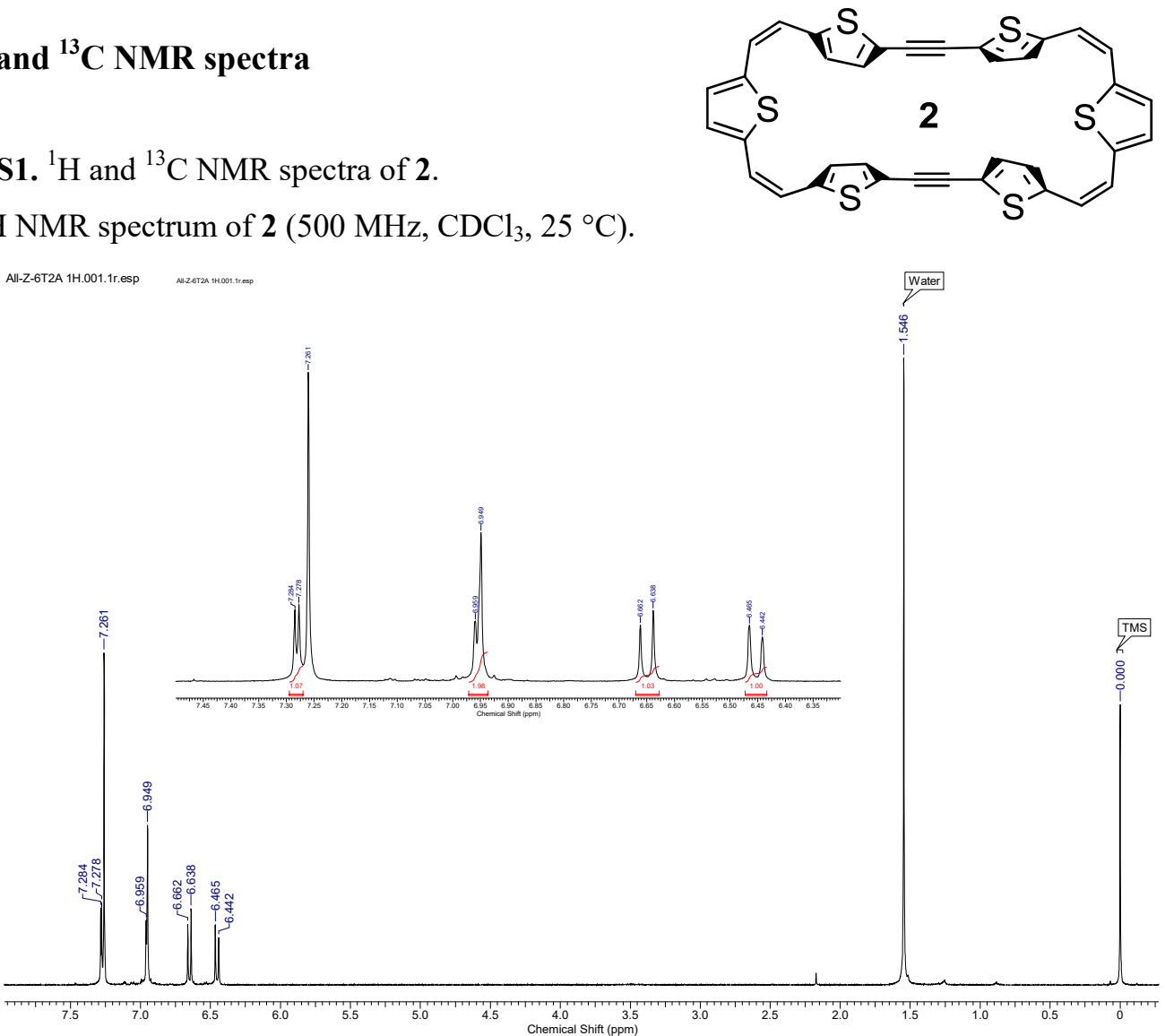

(b) ${ }^{13} \mathrm{C}$ NMR spectrum of $2\left(125 \mathrm{MHz}, \mathrm{CDCl}_{3}, 25^{\circ} \mathrm{C}\right)$.

All-Z-6T2A 13C.001.1r.esp

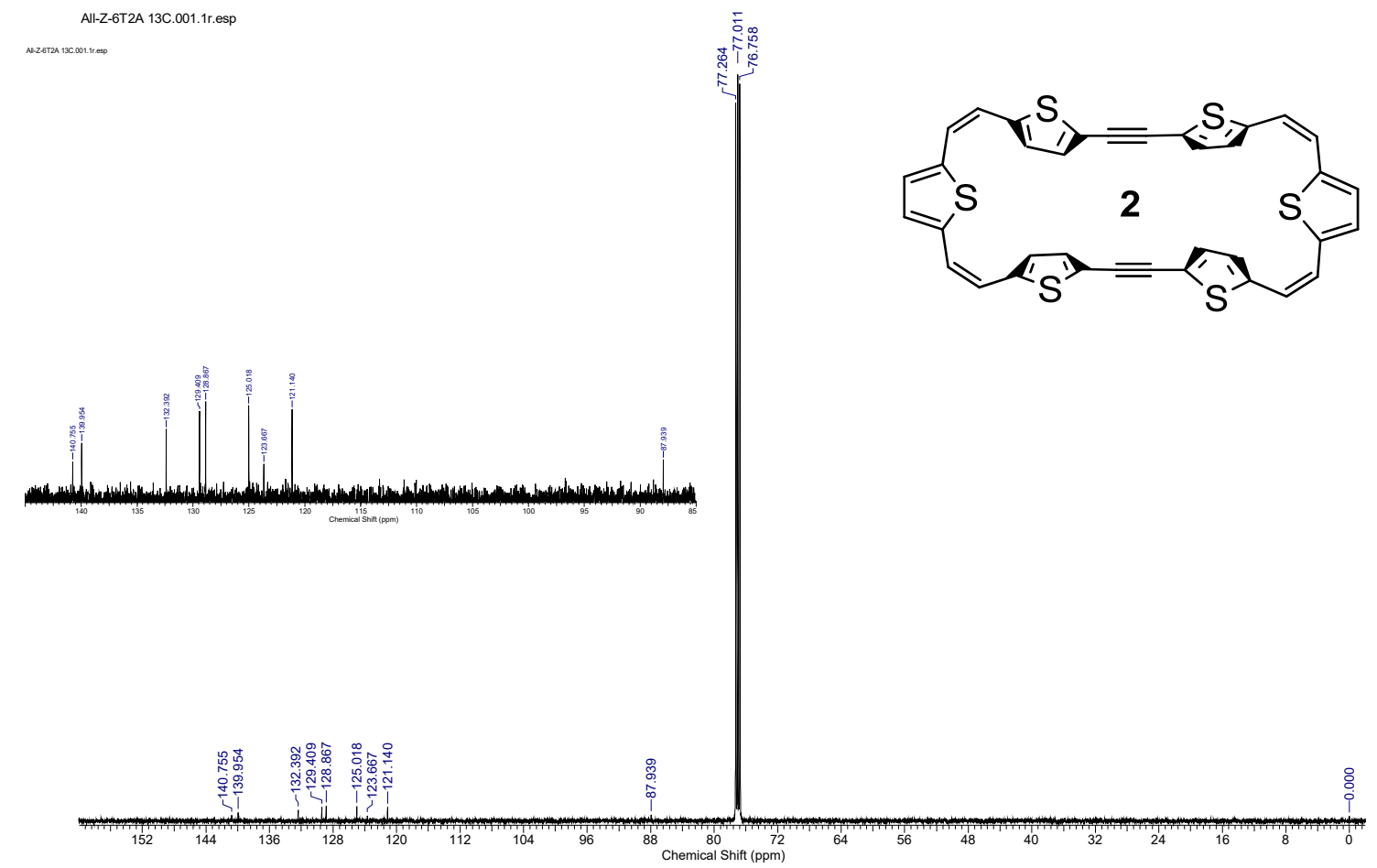


Figure S2. ${ }^{1} \mathrm{H}$ and ${ }^{13} \mathrm{C}$ NMR spectra of $\mathbf{3}$.

(a) ${ }^{1} \mathrm{H}$ NMR spectrum of $\mathbf{3}\left(500 \mathrm{MHz}\right.$, toluene- $\left.d_{8}, 25^{\circ} \mathrm{C}\right)$.

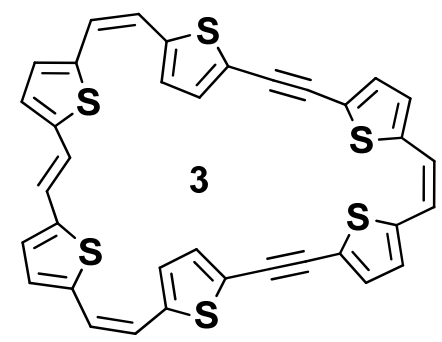

E,Z,Z,Z-6T2A 1H d-Toluene.001.1r.esp

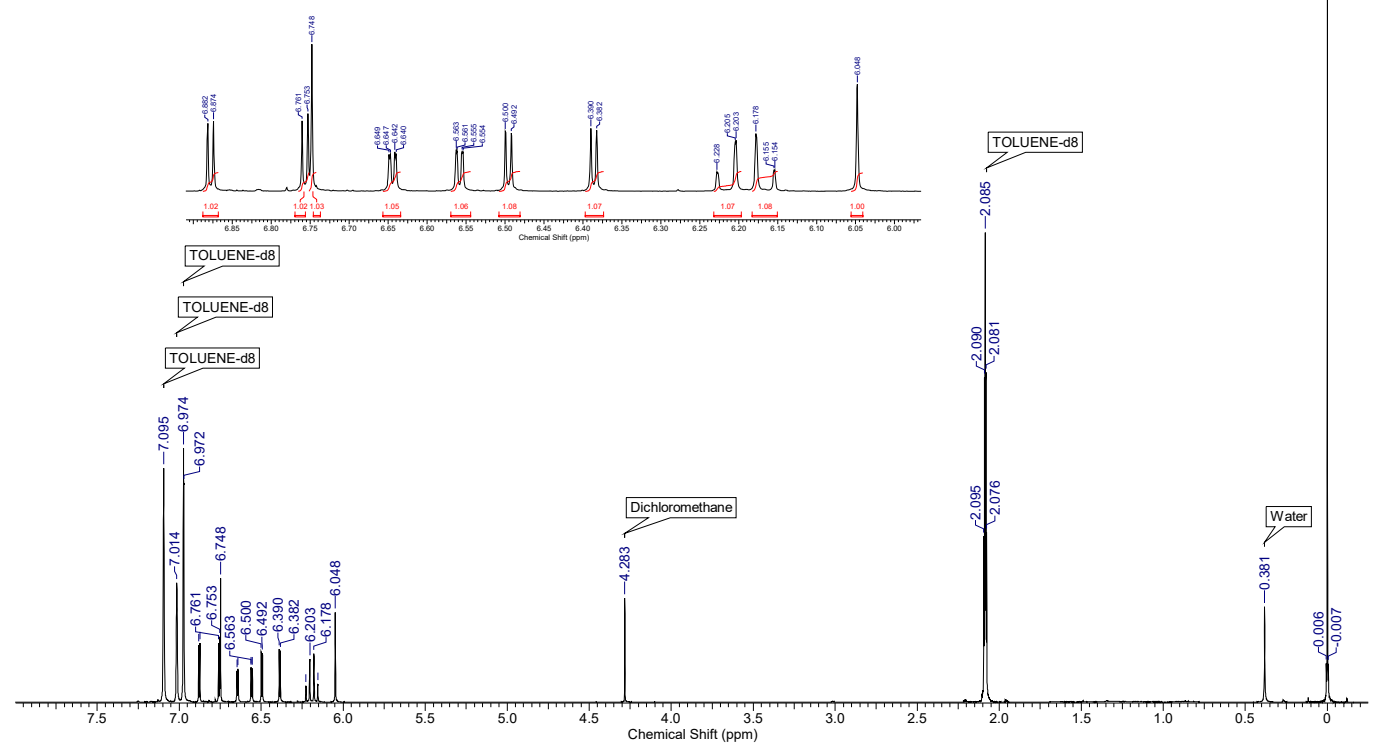

The ${ }^{1} \mathrm{H}$ NMR spectrum of 3 at $-60{ }^{\circ} \mathrm{C}$ exhibited almost no spectral change. Therefore, rapid rotation of $E$-double was concluded.

(b) ${ }^{13} \mathrm{C}$ NMR spectrum of $\mathbf{3}\left(125 \mathrm{MHz}, \mathrm{CDCl}_{3}, 25^{\circ} \mathrm{C}\right)$.

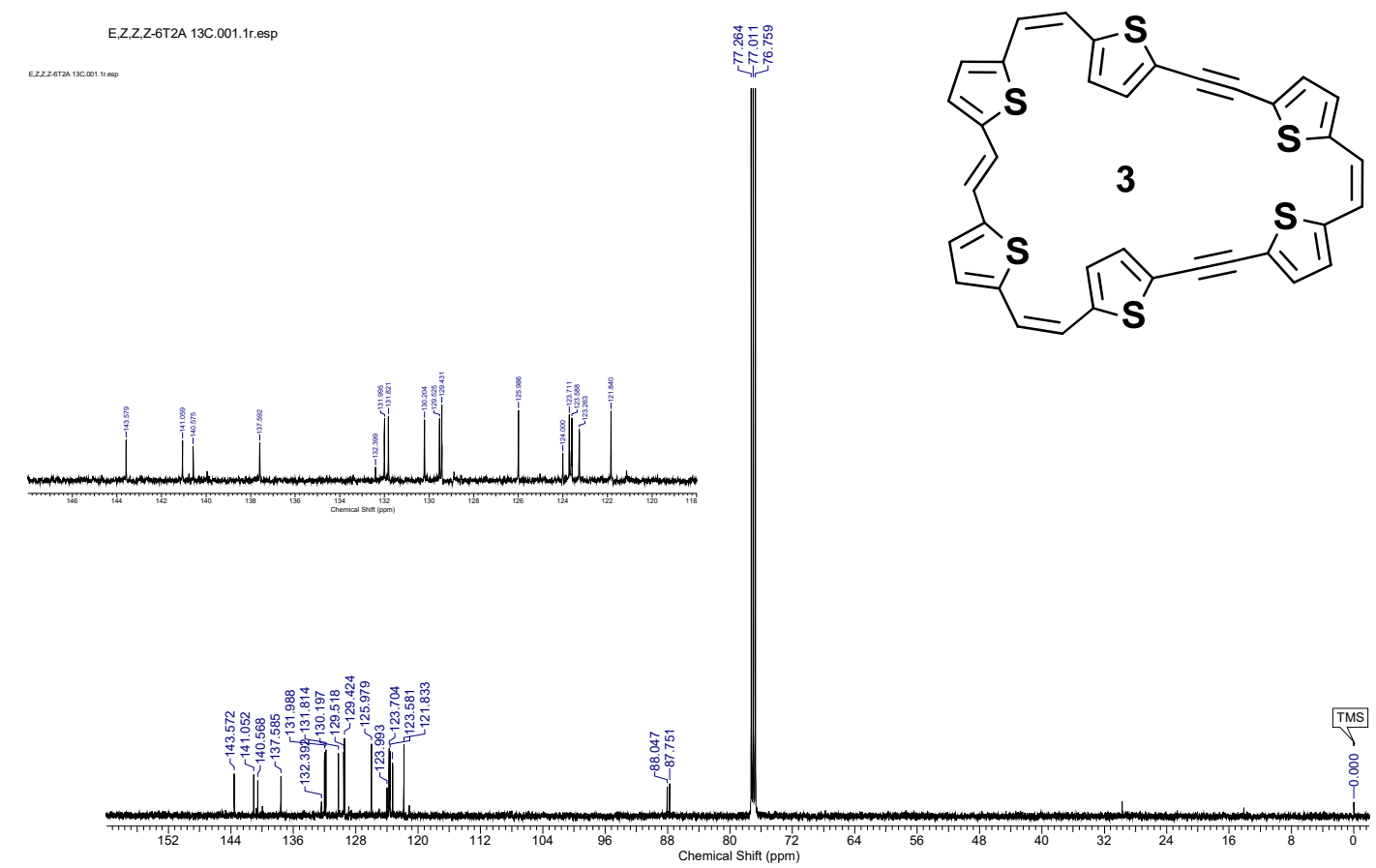


Figure S3. ${ }^{1} \mathrm{H}$ NMR spectrum of 4.

(a) ${ }^{1} \mathrm{H}$ NMR spectrum of $4\left(500 \mathrm{MHz}, \mathrm{CDCl}_{3}, 25^{\circ} \mathrm{C}\right)$.
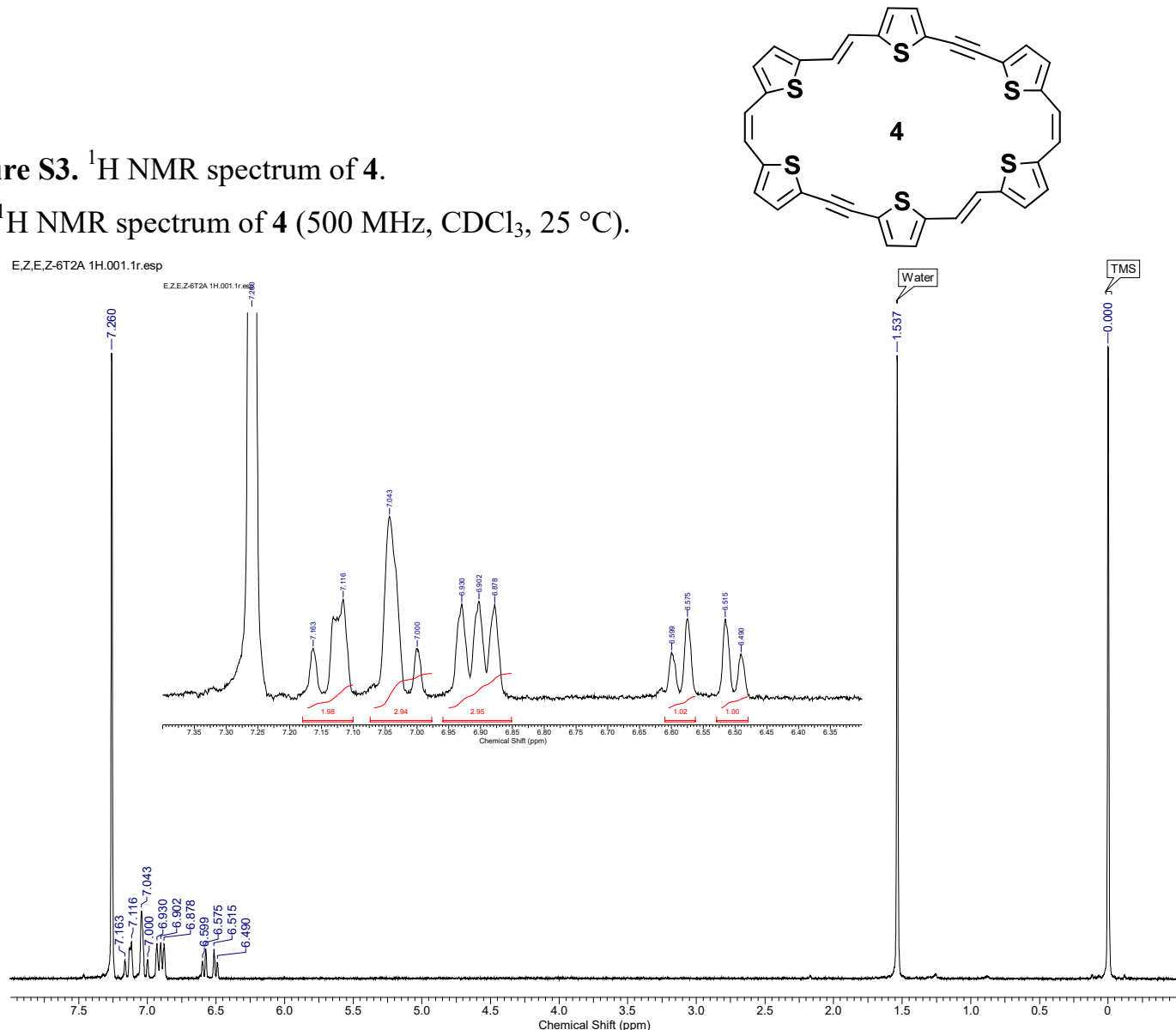

(b) ${ }^{1} \mathrm{H}$ NMR spectrum of 4 at $\delta 7.2 \sim 6.45\left(500 \mathrm{MHz}, \mathrm{CDCl}_{3}, 25^{\circ} \mathrm{C}\right)$.

Iff $\left[f^{\wedge} .093 .001 .1\right.$ r.esp

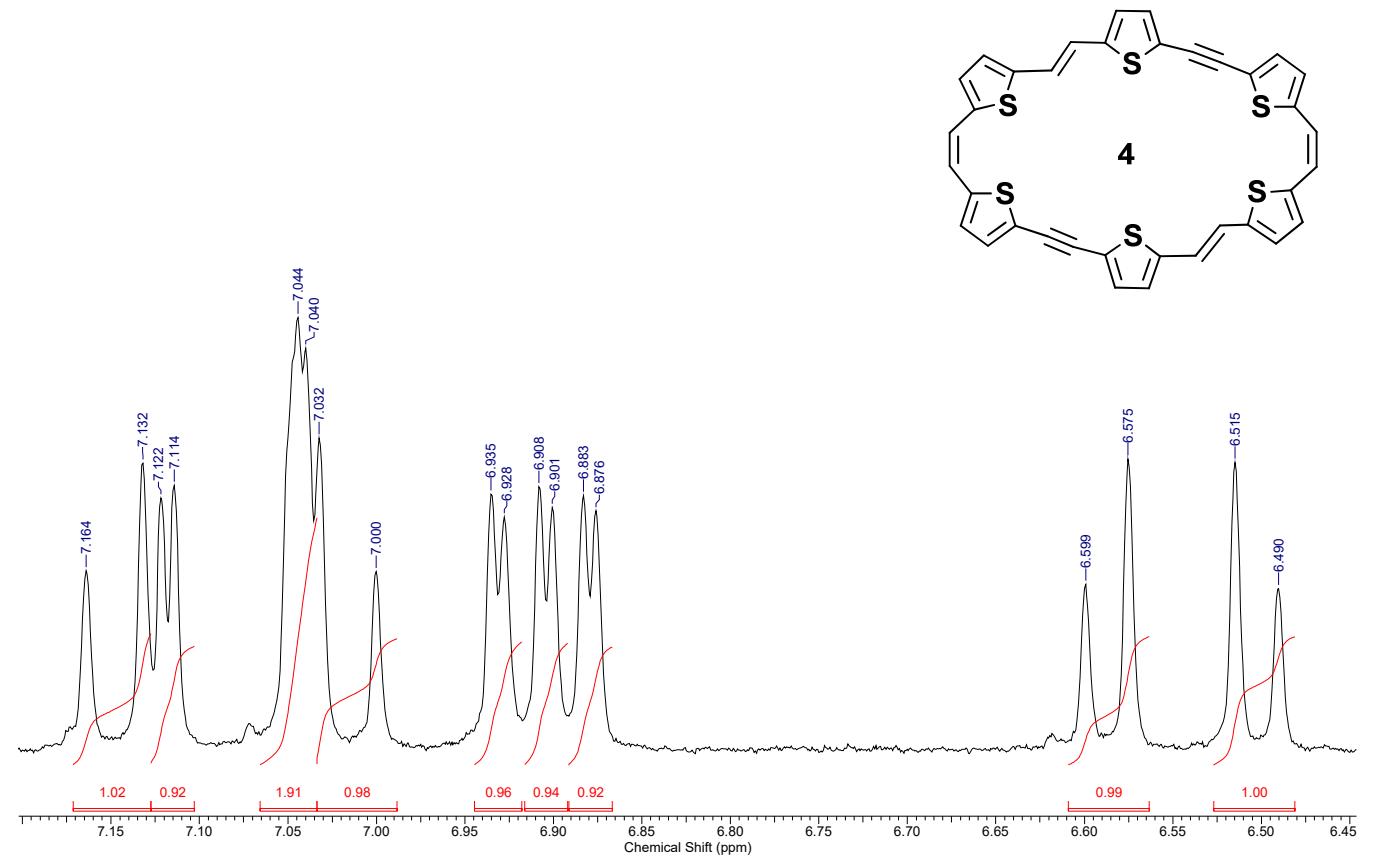

Because of very low solubility of $\mathbf{4},{ }^{13} \mathrm{C}$ NMR spectrum of $\mathbf{4}$ was not determined. 


\section{S2. X-ray crystallographic analysis of 2,3 , and 4}

Table S1. Detailed X-ray parameters of 2, 3, and 4.

\begin{tabular}{|c|c|c|c|}
\hline Identification code & 2 & 3 & 4 \\
\hline Crystal description & Orange prism & Orange plate & Dark red block \\
\hline Empirical formula & $\mathrm{C}_{36} \mathrm{H}_{20} \mathrm{~S}_{6}$ & $\mathrm{C}_{39} \mathrm{H}_{27} \mathrm{~S}_{6}$ & $\mathrm{C}_{39} \mathrm{H}_{23} \mathrm{~S}_{6}$ \\
\hline \multirow[t]{2}{*}{ Moiety formula } & $\mathrm{C}_{36} \mathrm{H}_{20} \mathrm{~S}_{6}$ & $\mathrm{C}_{36} \mathrm{H}_{20} \mathrm{~S}_{6}$ & $\mathrm{C}_{36} \mathrm{H}_{20} \mathrm{~S}_{6}$ \\
\hline & & $1 / 2\left(\mathrm{C}_{6} \mathrm{H}_{14}\right)$ & $1 / 2\left(\mathrm{C}_{6} \mathrm{H}_{6}\right)$ \\
\hline Formula weight & 644.88 & 687.96 & 683.93 \\
\hline Temperature & 93(2) K & 90 & $173 \mathrm{~K}$ \\
\hline Wavelength & $0.71075 \AA$ & $0.4139 \AA$ & $0.71073 \AA$ \\
\hline Crystal system & Triclinic & Monoclinic & Monoclinic \\
\hline Space group & $P-1(\# 2)$ & $P 2_{1} / n(\# 14)$ & $P 2_{1} / n(\# 14)$ \\
\hline \multirow[t]{6}{*}{ Unite cell dimensions } & $a=9.7254(10) \AA$ & $a=13.9308(12) \AA$ & $a=13.995(3) \AA$ \\
\hline & $b=11.0799(12) \AA$ & $b=12.8675(10) \AA$ & $b=12.223(3) \AA$ \\
\hline & $c=14.7514(14) \AA$ & $c=19.7859(16) \AA$ & $c=19.019(4) \AA$ \\
\hline & $\alpha=90.793(2)^{\circ}$ & $\alpha=90^{\circ}$ & $\alpha=90^{\circ}$ \\
\hline & $\beta=96.054(2)^{\circ}$ & $\beta=107.180(7)^{\circ}$ & $\beta=100.777(3)^{\circ}$ \\
\hline & $\gamma=106.886(2)^{\circ}$ & $\gamma=90^{\circ}$ & $\gamma=90^{\circ}$ \\
\hline Volume & $1510.9(3) \AA^{3}$ & $3388.5(5) \AA^{3}$ & $3196(1) \AA^{3}$ \\
\hline$Z$ & 2 & 4 & 4 \\
\hline Density (calc.) & $1.418 \mathrm{~g} / \mathrm{cm}^{3}$ & $1.349 \mathrm{~g} / \mathrm{cm}^{3}$ & $1.421 \mathrm{~g} / \mathrm{cm}^{3}$ \\
\hline Absorption coefficient & $0.479 \mathrm{~mm}^{-1}$ & $0.105 \mathrm{~mm}^{-1}$ & $0.457 \mathrm{~mm}^{-1}$ \\
\hline $\mathrm{F}(000)$ & 664 & 1428 & 1412 \\
\hline Crystal size & $0.16 \times 0.09 \times 0.09 \mathrm{~mm}^{3}$ & $0.2 \times 0.2 \times 0.03 \mathrm{~mm}^{3}$ & $0.2 \times 0.2 \times 0.1 \mathrm{~mm}^{3}$ \\
\hline \multirow[t]{3}{*}{ Index ranges } & $-12<=\mathrm{h}<=12$ & $-19<=\mathrm{h}<=19$ & $-10<=\mathrm{h}<=15$ \\
\hline & $-14<=\mathrm{k}<=14$ & $-17<=\mathrm{k}<=17$ & $-13<=\mathrm{k}<=11$ \\
\hline & $-19<=1<=16$ & $-27<=1<=27$ & $-21<=1<=19$ \\
\hline Reflections collected & 23888 & 88463 & 12687 \\
\hline Independent reflections & 6403 & 9512 & 4632 \\
\hline Data/restraints/parameters & $6403 / 0 / 379$ & $9512 / 36 / 465$ & $4632 / 0 / 395$ \\
\hline Goodness-of-fit on $\mathrm{F}^{2}$ & 1.039 & 1.148 & 1.017 \\
\hline Final $R$ indices & $R_{1}=0.0245$ & $R_{1}=0.0592$ & $R_{1}=0.0565$ \\
\hline$[\mathrm{I}>2 \sigma(\mathrm{I})]$ & $w R_{2}=0.0657$ & $w R_{2}=0.1445$ & $w R_{2}=0.1259$ \\
\hline Final $R$ indices & $R_{1}=0.0268$ & $R_{1}=0.0747$ & $R_{1}=0.0976$ \\
\hline [all data] & $w R_{2}=0.0667$ & $w R_{2}=0.1530$ & $w R_{2}=0.1480$ \\
\hline CCDC deposition No. & 1997209 & 1998429 & 1996534 \\
\hline
\end{tabular}


Figure SA. ORTEP drawing of 2 with atomic displacement ellipsoids at $50 \%$ probability level (green: carbon, yellow: sulfur; $\mathrm{H}$ atoms with arbitrary radii).

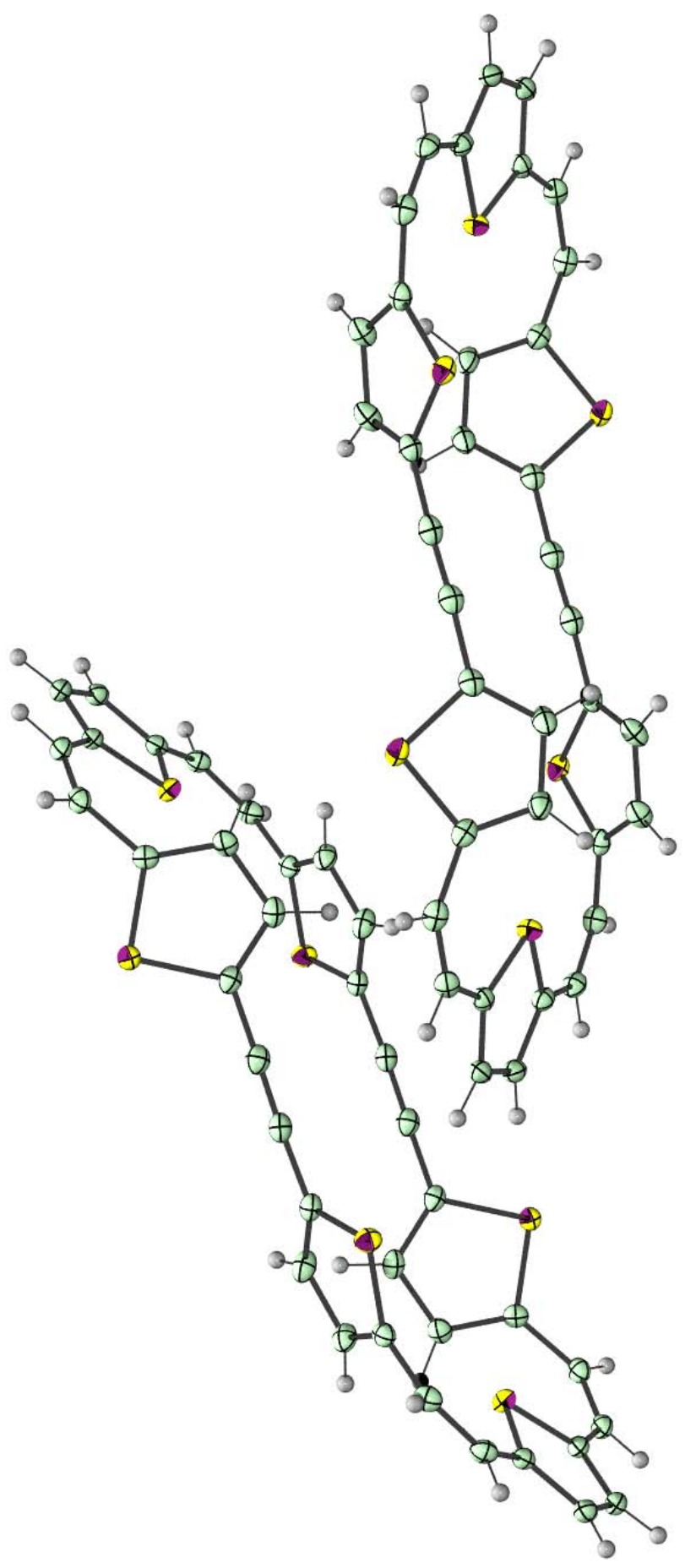


Figure SB. ORTEP drawing of $\mathbf{3}$ with atomic displacement ellipsoids at $50 \%$ probability level (green: carbon, yellow: sulfur; $\mathrm{H}$ atoms with arbitrary radii). Disordered part is omitted for clarity.

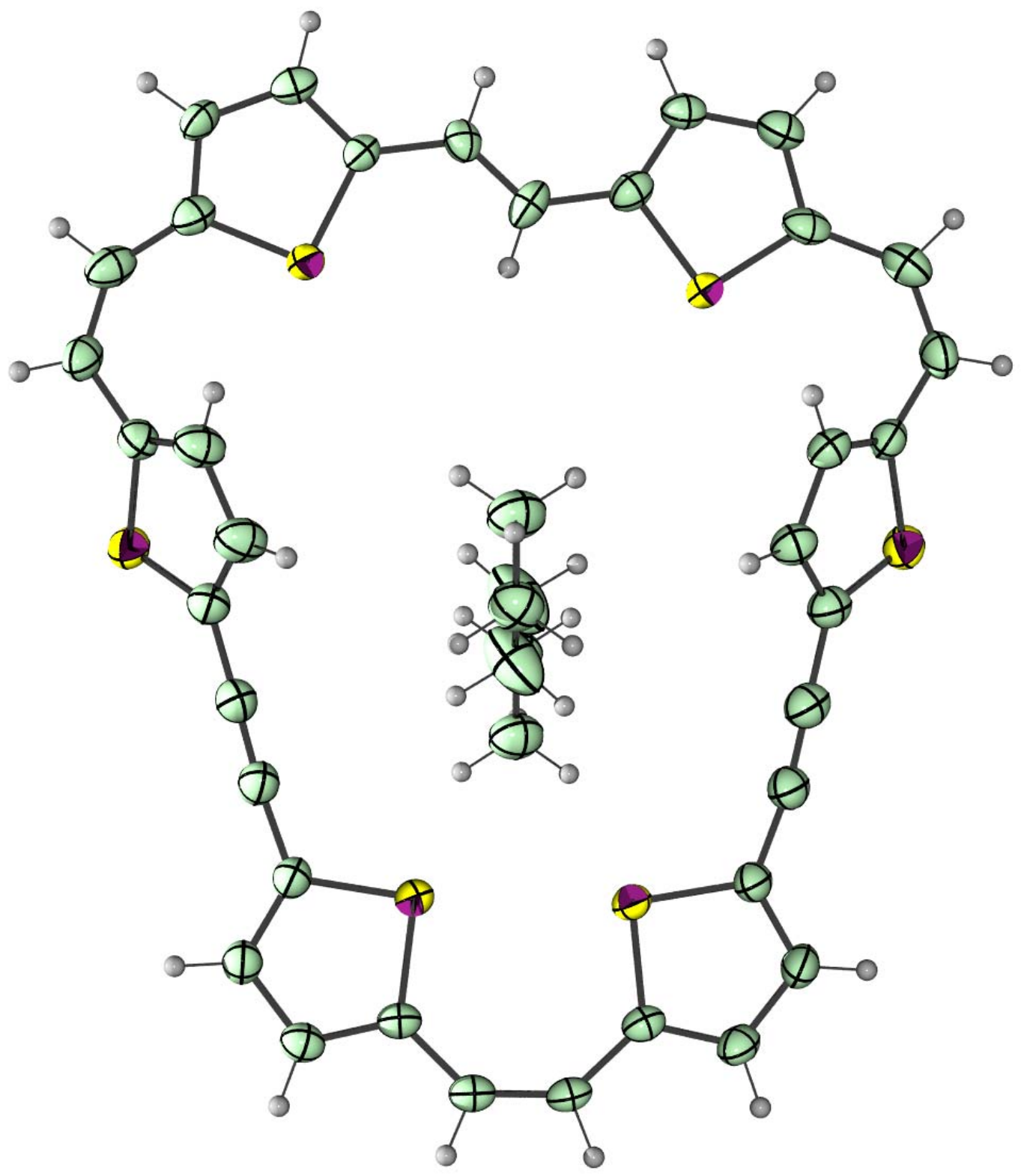


Figure SC. RTEP drawing of 4 with atomic displacement ellipsoids at 50\% probability level (green: carbon, yellow: sulfur; $\mathrm{H}$ atoms with arbitrary radii). Disordered part is omitted for clarity.

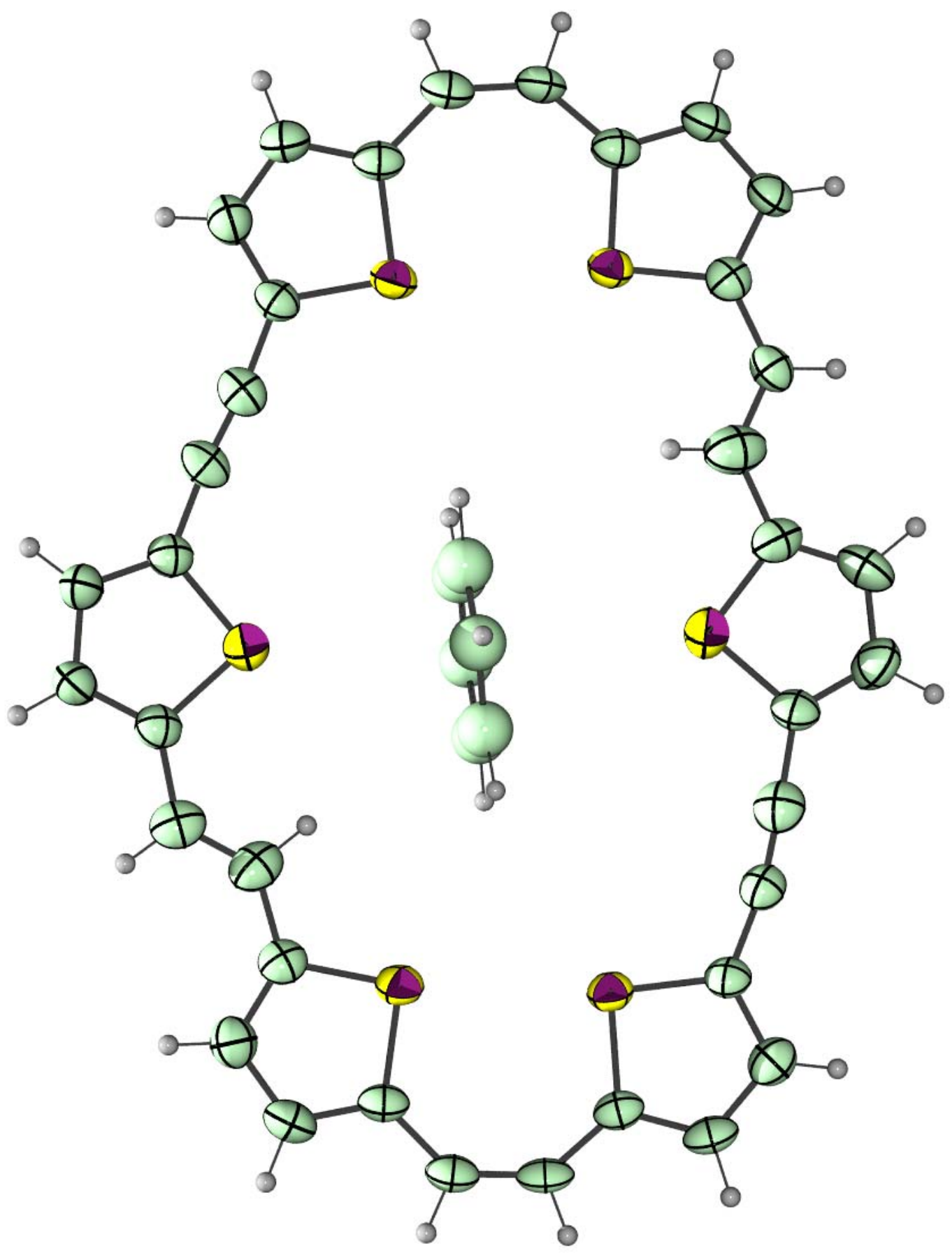




\section{S3. Geometrical optimization of 2,3 , and 4}

\section{S3-1. Geometrical optimization of 2.}

As for $\mathbf{2}$, there are two possible orientations of thiophene for the macroring: inward and outward. We started with two possible classifications of the thiophene orientations: $\boldsymbol{2}_{\text {out }}$ and $\boldsymbol{2}_{\text {in }}$ (Figure S1). Conformer $\mathbf{2}_{\text {out }}$ contains four outward thiophenes and two inward side thiophene rings. The other conformer $\mathbf{2}_{\text {in }}$ is composed of inward thiophenes.

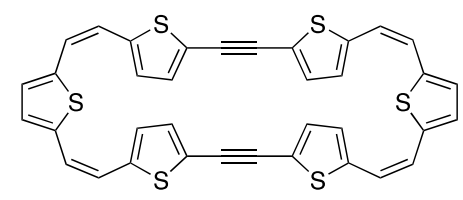

$2_{\text {out }}$

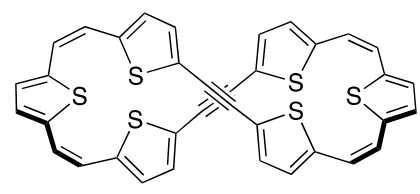

$2_{\text {in }}$

Figure S4. Possible conformations of 2.

Finally, we found four stable conformers of macroring with four outward thiophene rings: $\boldsymbol{2}_{\text {out }}$ $\left(C_{2}\right), \boldsymbol{2}_{\text {out }}$-ii $\left(C_{1}\right), \boldsymbol{2}_{\text {out }}$-iii $\left(C_{2}\right)$, and $\mathbf{2}_{\text {out }}$-iv $\left(C_{1}\right)$ with local minimum energy. They are conformers with different orientation of opposing thiophene in the macroring (Table S2). Conformer of $\mathbf{2}$-i has similar geometry to the molecular structure obtained from X-ray analysis. Other conformers and their energies are listed in Table S2.

In case of $\boldsymbol{2}_{\text {in }}$, we obtained a twisted figure-eight geometry was found with $D_{2}$ symmetry.

Table S2. Geometry and total energy of each conformer in $\mathbf{2}$.

\begin{tabular}{|c|c|c|c|c|}
\hline ID code & out $-\mathrm{X}-\mathrm{ray})$ \\
\hline Geometry
\end{tabular}




\begin{tabular}{|c|c|}
\hline ID code & \\
\hline Geometry \\
\hline Point Group & $D_{2}$ \\
\hline Energy in HF & -3772.8206963 \\
\hline $\begin{array}{c}\text { Relative } \\
\text { energy }\end{array}$ & $+14.7 \mathrm{~kJ} / \mathrm{mol}$ \\
\hline$E_{\mathrm{LUMO}}$ & $-2.43 \mathrm{eV}$ \\
\hline$E_{\text {HOMO }}$ & $-4.65 \mathrm{eV}$ \\
\hline$\Delta E_{\text {HOMO-LUMO }}$ & $2.23 \mathrm{eV}$ \\
\hline
\end{tabular}

S3-2. Geometrical optimization of 3 .

To get started, we performed geometrical optimization and frequency calculations form a planar initial structure. We found three imaginary frequencies leading to other structures with lower energy. Finally, two independent conformers of 3-i $\left(C_{1}\right)$ and 3-ii $\left(C_{1}\right)$ having local minimum energy were obtained. These conformers are not planar geometry, and four side thiophene rings are twisted from $\pi$-plane of the macroring. In these conformers, two of six thiophene rings have same or different orientation (Table S3).

Table S3. Geometry and total energy of each conformer in $\mathbf{3}$.

\begin{tabular}{|c|c|c|}
\hline ID code & $3-\mathrm{i}$ & 3-ii (X-ray) \\
\hline Geometry & & \\
\hline Point Group & $C_{1}$ & $C_{1}$ \\
\hline Energy in HF & -3772.832293 & -3772.8318911 \\
\hline $\begin{array}{l}\text { Relative } \\
\text { energy }\end{array}$ & $0.00 \mathrm{~kJ} / \mathrm{mol}$ & $+1.06 \mathrm{~kJ} / \mathrm{mol}$ \\
\hline$E_{\mathrm{LUMO}}$ & $-2.52 \mathrm{eV}$ & $-2.49 \mathrm{eV}$ \\
\hline$E_{\mathrm{HOMO}}$ & $-4.73 \mathrm{eV}$ & $-4.76 \mathrm{eV}$ \\
\hline$\Delta E_{\text {номо-LUмо }}$ & $2.21 \mathrm{eV}$ & $2.27 \mathrm{eV}$ \\
\hline
\end{tabular}


S3-3. Geometrical optimization of 4.

In case of $\mathbf{4}$, there are two Z-olefin at the opposite positions. We start with three different conformations by the orientation of the olefin moieties. As a result, three planar structures of 4-i $\left(C_{2 \mathrm{~h}}\right), 4$-ii $\left(C_{\mathrm{s}}\right)$, and 4-iii $\left(C_{2 \mathrm{~h}}\right)$ having local minimum energy were obtained (Table S4).

Table S4. Geometry and total energy of each conformer in $\mathbf{4}$.

\begin{tabular}{|c|c|c|c|}
\hline ID code & $4-i$ & 4-ii (X-ray) & 4-iii \\
\hline Geometry & & & \\
\hline Point Group & $C_{2 \mathrm{~h}}$ & $C_{\mathrm{s}}$ & $C_{2 \mathrm{~h}}$ \\
\hline Energy in HF & -3772.8406298 & -3772.8392229 & -3772.8402303 \\
\hline $\begin{array}{l}\text { Relative } \\
\text { energy }\end{array}$ & $0.00 \mathrm{~kJ} / \mathrm{mol}$ & $+3.69 \mathrm{~kJ} / \mathrm{mol}$ & $+1.05 \mathrm{~kJ} / \mathrm{mol}$ \\
\hline$E_{\mathrm{LUMO}}$ & $-2.59 \mathrm{eV}$ & $-2.59 \mathrm{eV}$ & $-2.59 \mathrm{eV}$ \\
\hline$E_{\text {Номо }}$ & $-4.55 \mathrm{eV}$ & $-4.55 \mathrm{eV}$ & $-4.55 \mathrm{eV}$ \\
\hline$\Delta E_{\text {номо-LUмо }}$ & $1.97 \mathrm{eV}$ & $1.97 \mathrm{eV}$ & $1.97 \mathrm{eV}$ \\
\hline
\end{tabular}

S3-4. Cartesian coordinates at B3LYP/6-31G(d,p) level.

Table S5. Cartesian coordinate of optimized structure of $\boldsymbol{2}_{\text {out }}-i$ at B3LYP/6-31G(d,p) level.

\begin{tabular}{rrrrrr}
$\begin{array}{c}\text { Center } \\
\text { Number }\end{array}$ & $\begin{array}{c}\text { Atomic } \\
\text { Number }\end{array}$ & $\begin{array}{c}\text { Atomic } \\
\text { Type }\end{array}$ & X & Coordinates (Angstroms) & Z \\
\hline 1 & 16 & 0 & -1.215089 & 3.026697 & -3.358336 \\
2 & 16 & 0 & 0.028822 & 5.982586 & 0.030765 \\
3 & 16 & 0 & -0.112072 & 3.158332 & 3.772724 \\
4 & 6 & 0 & 0.047422 & 0.608336 & -2.908031 \\
5 & 6 & 0 & 0.137216 & 2.008463 & -2.880661 \\
6 & 6 & 0 & 1.206327 & 2.790879 & -2.478688 \\
7 & 1 & 0 & 2.147264 & 2.360973 & -2.157199 \\
8 & 6 & 0 & 0.945853 & 4.178604 & -2.543731 \\
9 & 1 & 0 & 1.677408 & 4.938796 & -2.299389 \\
10 & 6 & 0 & -0.323729 & 4.492500 & -2.989312 \\
11 & 6 & 0 & -0.912903 & 5.796262 & -3.262557 \\
12 & 1 & 0 & -1.444779 & 5.874595 & -4.209180 \\
13 & 6 & 0 & -0.874322 & 6.925083 & -2.511424 \\
14 & 1 & 0 & -1.305726 & 7.809745 & -2.977767 \\
15 & 6 & 0 & -0.432551 & 7.189337 & -1.159132 \\
16 & 6 & 0 & -0.432551 & 8.448320 & -0.580510 \\
17 & 1 & 0 & -0.700966 & 9.340310 & -1.136214 \\
18 & 6 & 0 & -0.075565 & 8.450134 & 0.782898 \\
19 & 1 & 0 & -0.037264 & 9.343852 & 1.396224
\end{tabular}




\begin{tabular}{|c|c|c|c|c|c|}
\hline 20 & 6 & 0 & 0.195885 & 7.191272 & 1.295903 \\
\hline 21 & 6 & 0 & 0.469315 & 6.947403 & 2.695559 \\
\hline 22 & 1 & 0 & 0.461041 & 7.871178 & 3.272439 \\
\hline 23 & 6 & 0 & 0.646259 & 5.814941 & 3.421592 \\
\hline 24 & 1 & 0 & 0.689420 & 5.946402 & 4.501343 \\
\hline 25 & 6 & 0 & 0.762830 & 4.440649 & 2.954818 \\
\hline 26 & 6 & 0 & 1.532920 & 3.918507 & 1.934824 \\
\hline 27 & 1 & 0 & 2.189240 & 4.532529 & 1.331178 \\
\hline 28 & 6 & 0 & 1.411936 & 2.518141 & 1.783135 \\
\hline 29 & 1 & 0 & 1.930539 & 1.937915 & 1.030026 \\
\hline 30 & 6 & 0 & 0.552993 & 1.935481 & 2.696570 \\
\hline 31 & 6 & 0 & 0.169827 & 0.585735 & 2.769764 \\
\hline 32 & 16 & 0 & 0.112072 & -3.158332 & 3.772724 \\
\hline 33 & 16 & 0 & -0.028822 & -5.982586 & 0.030765 \\
\hline 34 & 16 & 0 & 1.215089 & -3.026697 & -3.358336 \\
\hline 35 & 6 & 0 & -0.169827 & -0.585735 & 2.769764 \\
\hline 36 & 6 & 0 & -0.552993 & -1.935481 & 2.696570 \\
\hline 37 & 6 & 0 & -1.411936 & -2.518141 & 1.783135 \\
\hline 38 & 1 & 0 & -1.930539 & -1.937915 & 1.030026 \\
\hline 39 & 6 & 0 & -1.532920 & -3.918507 & 1.934824 \\
\hline 40 & 1 & 0 & -2.189240 & -4.532529 & 1.331178 \\
\hline 41 & 6 & 0 & -0.762830 & -4.440649 & 2.954818 \\
\hline 42 & 6 & 0 & -0.646259 & -5.814941 & 3.421592 \\
\hline 43 & 1 & 0 & -0.689420 & -5.946402 & 4.501343 \\
\hline 44 & 6 & 0 & -0.469315 & -6.947403 & 2.695559 \\
\hline 45 & 1 & 0 & -0.461041 & -7.871178 & 3.272439 \\
\hline 46 & 6 & 0 & -0.195885 & -7.191272 & 1.295903 \\
\hline 47 & 6 & 0 & 0.075565 & -8.450134 & 0.782898 \\
\hline 48 & 1 & 0 & 0.037264 & -9.343852 & 1.396224 \\
\hline 49 & 6 & 0 & 0.432551 & -8.448320 & -0.580510 \\
\hline 50 & 1 & 0 & 0.700966 & -9.340310 & -1.136214 \\
\hline 51 & 6 & 0 & 0.432551 & -7.189337 & -1.159132 \\
\hline 52 & 6 & 0 & 0.874322 & -6.925083 & -2.511424 \\
\hline 53 & 1 & 0 & 1.305726 & -7.809745 & -2.977767 \\
\hline 54 & 6 & 0 & 0.912903 & -5.796262 & -3.262557 \\
\hline 55 & 1 & 0 & 1.444779 & -5.874595 & -4.209180 \\
\hline 56 & 6 & 0 & 0.323729 & -4.492500 & -2.989312 \\
\hline 57 & 6 & 0 & -0.945853 & -4.178604 & -2.543731 \\
\hline 58 & 1 & 0 & -1.677408 & -4.938796 & -2.299389 \\
\hline 59 & 6 & 0 & -1.206327 & -2.790879 & -2.478688 \\
\hline 60 & 1 & 0 & -2.147264 & -2.360973 & -2.157199 \\
\hline 61 & 6 & 0 & -0.137216 & -2.008463 & -2.880661 \\
\hline 62 & 6 & 0 & -0.047422 & -0.608336 & -2.908031 \\
\hline
\end{tabular}

Compound: $\boldsymbol{2}_{\text {out }} \mathrm{-}$ (See Table S2)

Method: B3LYP/6-31G(d,p)

Key word: opt freq $\mathrm{scf}=($ direct,tight $)$

Symmetry: $C_{2}$

\# of imaginary frequencies: 0

Energy: -3772.8220326 Hartree

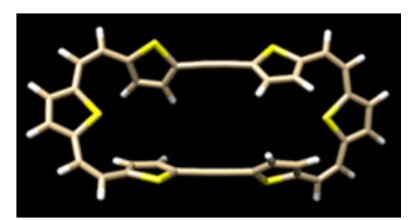

Table S6. Cartesian coordinate of optimized structure of $\boldsymbol{2}_{\text {out }}$-ii at B3LYP/6-31G(d,p) level.

\begin{tabular}{cccccc}
$\begin{array}{c}\text { Center } \\
\text { Number }\end{array}$ & $\begin{array}{c}\text { Atomic } \\
\text { Number }\end{array}$ & $\begin{array}{c}\text { Atomic } \\
\text { Type }\end{array}$ & X & Y Coordinates (Angstroms) & Z \\
\hline 1 & 6 & 0 & 7.100369 & 1.267529 & -0.649590 \\
2 & 6 & 0 & 8.319661 & 0.703957 & -0.992226 \\
3 & 6 & 0 & 8.319495 & -0.704836 & -0.992109 \\
4 & 6 & 0 & 7.100074 & -1.268071 & -0.649369 \\
5 & 16 & 0 & 5.932864 & -0.000104 & -0.303716
\end{tabular}




\begin{tabular}{|c|c|c|c|c|c|}
\hline 6 & 1 & 0 & 9.181463 & 1.308072 & -1.254424 \\
\hline 7 & 1 & 0 & 9.181155 & -1.309196 & -1.254209 \\
\hline 8 & 6 & 0 & 6.839752 & -2.689857 & -0.695588 \\
\hline 9 & 1 & 0 & 7.700207 & -3.242933 & -1.069573 \\
\hline 10 & 6 & 0 & 6.840355 & 2.689362 & -0.696029 \\
\hline 11 & 1 & 0 & 7.700935 & 3.242220 & -1.070050 \\
\hline 12 & 6 & 0 & 5.738102 & -3.441551 & -0.442341 \\
\hline 13 & 1 & 0 & 5.812098 & -4.492481 & -0.716553 \\
\hline 14 & 6 & 0 & 5.738825 & 3.441268 & -0.442919 \\
\hline 15 & 1 & 0 & 5.812978 & 4.492159 & -0.717240 \\
\hline 16 & 6 & 0 & 4.466850 & -3.043561 & 0.141044 \\
\hline 17 & 6 & 0 & 4.207158 & -2.249530 & 1.241807 \\
\hline 18 & 16 & 0 & 2.967081 & -3.686583 & -0.508573 \\
\hline 19 & 6 & 0 & 2.835518 & -2.132975 & 1.557001 \\
\hline 20 & 1 & 0 & 4.996527 & -1.790325 & 1.823417 \\
\hline 21 & 6 & 0 & 2.009860 & -2.846532 & 0.703414 \\
\hline 22 & 1 & 0 & 2.446240 & -1.551867 & 2.383863 \\
\hline 23 & 6 & 0 & 4.467498 & 3.043468 & 0.140447 \\
\hline 24 & 6 & 0 & 4.207716 & 2.249629 & 1.241325 \\
\hline 25 & 16 & 0 & 2.967781 & 3.686282 & -0.509489 \\
\hline 26 & 6 & 0 & 2.836055 & 2.133204 & 1.556479 \\
\hline 27 & 1 & 0 & 4.997042 & 1.790490 & 1.823048 \\
\hline 28 & 6 & 0 & 2.010475 & 2.846646 & 0.702722 \\
\hline 29 & 1 & 0 & 2.446709 & 1.552268 & 2.383429 \\
\hline 30 & 6 & 0 & 0.610627 & 2.899575 & 0.705740 \\
\hline 31 & 6 & 0 & -0.610011 & 2.899548 & 0.705657 \\
\hline 32 & 6 & 0 & -2.009863 & 2.846628 & 0.702575 \\
\hline 33 & 16 & 0 & -2.967134 & 3.686507 & -0.509494 \\
\hline 34 & 6 & 0 & -2.835483 & 2.133123 & 1.556243 \\
\hline 35 & 6 & 0 & -4.466879 & 3.043590 & 0.140284 \\
\hline 36 & 6 & 0 & -4.207133 & 2.249609 & 1.241071 \\
\hline 37 & 1 & 0 & -2.446166 & 1.552079 & 2.383132 \\
\hline 38 & 6 & 0 & -5.738158 & 3.441513 & -0.443084 \\
\hline 39 & 1 & 0 & -4.996475 & 1.790427 & 1.822736 \\
\hline 40 & 6 & 0 & -6.839817 & 2.689775 & -0.696151 \\
\hline 41 & 1 & 0 & -5.812165 & 4.492399 & -0.717459 \\
\hline 42 & 6 & 0 & -7.100106 & 1.267996 & -0.649660 \\
\hline 43 & 1 & 0 & -7.700300 & 3.242774 & -1.070188 \\
\hline 44 & 6 & 0 & -8.319514 & 0.704661 & -0.992281 \\
\hline 45 & 16 & 0 & -5.932856 & 0.000133 & -0.303758 \\
\hline 46 & 6 & 0 & -8.319640 & -0.704131 & -0.992110 \\
\hline 47 & 1 & 0 & -9.181193 & 1.308944 & -1.254496 \\
\hline 48 & 6 & 0 & -7.100337 & -1.267601 & -0.649345 \\
\hline 49 & 1 & 0 & -9.181427 & -1.308323 & -1.254180 \\
\hline 50 & 6 & 0 & -6.840298 & -2.689441 & -0.695481 \\
\hline 51 & 1 & 0 & -7.700861 & -3.242384 & -1.069415 \\
\hline 52 & 6 & 0 & -5.738777 & -3.441294 & -0.442179 \\
\hline 53 & 1 & 0 & -5.812928 & -4.492242 & -0.716281 \\
\hline 54 & 6 & 0 & -4.467470 & -3.043397 & 0.141169 \\
\hline 55 & 6 & 0 & -4.207733 & -2.249442 & 1.241973 \\
\hline 56 & 16 & 0 & -2.967742 & -3.686403 & -0.508543 \\
\hline 57 & 6 & 0 & -2.836081 & -2.132967 & 1.557158 \\
\hline 58 & 1 & 0 & -4.997081 & -1.790238 & 1.823614 \\
\hline 59 & 6 & 0 & -2.010468 & -2.846490 & 0.703502 \\
\hline 60 & 1 & 0 & -2.446766 & -1.551937 & 2.384057 \\
\hline 61 & 6 & 0 & 0.610013 & -2.899470 & 0.706570 \\
\hline 62 & 6 & 0 & -0.610622 & -2.899470 & 0.706607 \\
\hline
\end{tabular}

Compound: $\boldsymbol{2}_{\text {out }}$-ii (See Table S2)

Method: B3LYP/6-31G(d,p)

Key word: opt freq scf=(direct,tight)

Symmetry: $C_{1}$

\# of imaginary frequencies: 0

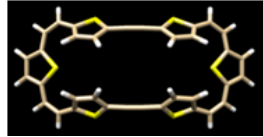

Energy: -3772.8245752 Hartree 
Table S7. Cartesian coordinate of optimized structure of $\boldsymbol{2}_{\text {out }}$-iii at B3LYP/6-31G(d,p) level.

\begin{tabular}{|c|c|c|c|c|c|}
\hline \multirow{2}{*}{$\begin{array}{l}\text { Center } \\
\text { Number }\end{array}$} & \multirow{2}{*}{$\begin{array}{l}\text { Atomic } \\
\text { Number }\end{array}$} & \multirow{2}{*}{$\begin{array}{l}\text { Atomic } \\
\text { Type }\end{array}$} & \multicolumn{3}{|c|}{ Coordinates (Angstroms) } \\
\hline & & & $\mathrm{X}$ & $\mathrm{Y}$ & $\mathrm{Z}$ \\
\hline 1 & 6 & 0 & -0.359117 & -1.214437 & -7.183020 \\
\hline 2 & 6 & 0 & -0.209822 & -0.673582 & -8.449132 \\
\hline 3 & 6 & 0 & 0.209822 & 0.673582 & -8.449132 \\
\hline 4 & 6 & 0 & 0.359117 & 1.214437 & -7.183020 \\
\hline 5 & 16 & 0 & 0.000000 & 0.000000 & -5.965425 \\
\hline 6 & 1 & 0 & -0.402909 & -1.246552 & -9.349615 \\
\hline 7 & 1 & 0 & 0.402909 & 1.246552 & -9.349615 \\
\hline 8 & 6 & 0 & 0.868838 & 2.544392 & -6.921160 \\
\hline 9 & 1 & 0 & 1.304873 & 2.999415 & -7.809366 \\
\hline 10 & 6 & 0 & -0.868838 & -2.544392 & -6.921160 \\
\hline 11 & 1 & 0 & -1.304873 & -2.999415 & -7.809366 \\
\hline 12 & 6 & 0 & 0.943954 & 3.286249 & -5.788119 \\
\hline 13 & 1 & 0 & 1.512312 & 4.211736 & -5.862647 \\
\hline 14 & 6 & 0 & -0.943954 & -3.286249 & -5.788119 \\
\hline 15 & 1 & 0 & -1.512312 & -4.211736 & -5.862647 \\
\hline 16 & 6 & 0 & 0.339626 & 3.031551 & -4.488270 \\
\hline 17 & 6 & 0 & -0.934565 & 2.591295 & -4.184549 \\
\hline 18 & 16 & 0 & 1.215090 & 3.413487 & -3.015851 \\
\hline 19 & 6 & 0 & -1.210036 & 2.536907 & -2.800278 \\
\hline 20 & 1 & 0 & -1.657218 & 2.336025 & -4.949387 \\
\hline 21 & 6 & 0 & -0.149373 & 2.944410 & -2.009385 \\
\hline 22 & 1 & 0 & -2.152757 & 2.210645 & -2.378474 \\
\hline 23 & 6 & 0 & -0.339626 & -3.031551 & -4.488270 \\
\hline 24 & 6 & 0 & 0.934565 & -2.591295 & -4.184549 \\
\hline 25 & 16 & 0 & -1.215090 & -3.413487 & -3.015851 \\
\hline 26 & 6 & 0 & 1.210036 & -2.536907 & -2.800278 \\
\hline 27 & 1 & 0 & 1.657218 & -2.336025 & -4.949387 \\
\hline 28 & 6 & 0 & 0.149373 & -2.944410 & -2.009385 \\
\hline 29 & 1 & 0 & 2.152757 & -2.210645 & -2.378474 \\
\hline 30 & 6 & 0 & -0.076184 & 2.978394 & -0.608987 \\
\hline 31 & 6 & 0 & 0.076184 & -2.978394 & -0.608987 \\
\hline 32 & 6 & 0 & -0.000000 & -2.979379 & 0.608951 \\
\hline 33 & 6 & 0 & -0.074102 & -2.947270 & 2.009345 \\
\hline 34 & 16 & 0 & 1.301657 & -3.381755 & 3.015952 \\
\hline 35 & 6 & 0 & -1.144800 & -2.566669 & 2.800127 \\
\hline 36 & 6 & 0 & 0.416684 & -3.022002 & 4.488282 \\
\hline 37 & 6 & 0 & -0.868193 & -2.614080 & 4.184423 \\
\hline 38 & 1 & 0 & -2.095416 & -2.264324 & 2.378223 \\
\hline 39 & 6 & 0 & 1.027195 & -3.261237 & 5.788173 \\
\hline 40 & 1 & 0 & -1.597145 & -2.377142 & 4.949180 \\
\hline 41 & 6 & 0 & 0.933179 & -2.521507 & 6.921201 \\
\hline 42 & 1 & 0 & 1.618900 & -4.171968 & 5.862759 \\
\hline 43 & 6 & 0 & 0.389840 & -1.204927 & 7.183028 \\
\hline 44 & 1 & 0 & 1.380588 & -2.965296 & 7.809433 \\
\hline 45 & 6 & 0 & 0.226846 & -0.668037 & 8.449141 \\
\hline 46 & 16 & 0 & -0.000000 & -0.000000 & 5.965430 \\
\hline 47 & 6 & 0 & -0.226846 & 0.668037 & 8.449141 \\
\hline 48 & 1 & 0 & 0.434413 & -1.235924 & 9.349623 \\
\hline 49 & 6 & 0 & -0.389840 & 1.204927 & 7.183028 \\
\hline 50 & 1 & 0 & -0.434413 & 1.235924 & 9.349623 \\
\hline 51 & 6 & 0 & -0.933179 & 2.521507 & 6.921201 \\
\hline 52 & 1 & 0 & -1.380588 & 2.965296 & 7.809433 \\
\hline 53 & 6 & 0 & -1.027195 & 3.261237 & 5.788173 \\
\hline 54 & 1 & 0 & -1.618900 & 4.171968 & 5.862759 \\
\hline 55 & 6 & 0 & -0.416684 & 3.022002 & 4.488282 \\
\hline 56 & 6 & 0 & 0.868193 & 2.614080 & 4.184423 \\
\hline 57 & 16 & 0 & -1.301657 & 3.381755 & 3.015952 \\
\hline 58 & 6 & 0 & 1.144800 & 2.566669 & 2.800127 \\
\hline 59 & 1 & 0 & 1.597145 & 2.377142 & 4.949180 \\
\hline
\end{tabular}




$\begin{array}{llllll}60 & 6 & 0 & 0.074102 & 2.947270 & 2.009345 \\ 61 & 1 & 0 & 2.095416 & 2.264324 & 2.378223 \\ 62 & 6 & 0 & 0.000000 & 2.979379 & 0.608951\end{array}$

Compound: $\boldsymbol{2}_{\text {out }}$-iii (See Table S2) Method: B3LYP/6-31G(d,p)

Key word: opt freq $\mathrm{scf}=($ direct,tight $)$

Symmetry: $C_{2}$

\# of imaginary frequencies: 0

Energy: -3772.8234752 Hartree

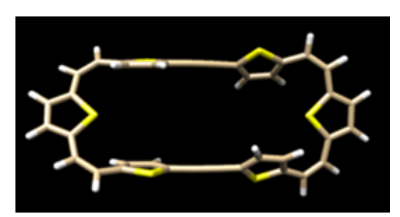

Table S8. Cartesian coordinate of optimized structure of $\boldsymbol{2}_{\text {out }}$-iv at B3LYP/6-31G(d,p) level.

\begin{tabular}{|c|c|c|c|c|c|}
\hline \multirow{2}{*}{$\begin{array}{l}\text { Center } \\
\text { Number }\end{array}$} & \multirow{2}{*}{$\begin{array}{l}\text { Atomic } \\
\text { Number }\end{array}$} & \multirow{2}{*}{$\begin{array}{l}\text { Atomic } \\
\text { Type }\end{array}$} & \multicolumn{3}{|c|}{ Coordinates (Angstroms) } \\
\hline & & & $\mathrm{X}$ & $\mathrm{Y}$ & $\mathrm{Z}$ \\
\hline 1 & 6 & 0 & 7.193269 & -1.272343 & 0.372314 \\
\hline 2 & 6 & 0 & 8.432864 & -0.701890 & 0.615513 \\
\hline 3 & 6 & 0 & 8.421155 & 0.706627 & 0.632652 \\
\hline 4 & 6 & 0 & 7.172898 & 1.262563 & 0.398849 \\
\hline 5 & 16 & 0 & 5.990682 & -0.012353 & 0.138087 \\
\hline 6 & 1 & 0 & 9.318454 & -1.301162 & 0.797145 \\
\hline 7 & 1 & 0 & 9.296588 & 1.315879 & 0.829644 \\
\hline 8 & 6 & 0 & 6.905436 & 2.680908 & 0.487750 \\
\hline 9 & 1 & 0 & 7.782852 & 3.234223 & 0.819542 \\
\hline 10 & 6 & 0 & 6.950747 & -2.697027 & 0.425475 \\
\hline 11 & 1 & 0 & 7.851643 & -3.247296 & 0.693507 \\
\hline 12 & 6 & 0 & 5.785275 & 3.428403 & 0.314574 \\
\hline 13 & 1 & 0 & 5.867825 & 4.473930 & 0.606356 \\
\hline 14 & 6 & 0 & 5.833263 & -3.453624 & 0.287541 \\
\hline 15 & 1 & 0 & 5.944646 & -4.508389 & 0.532823 \\
\hline 16 & 6 & 0 & 4.488372 & 3.035019 & -0.209924 \\
\hline 17 & 6 & 0 & 4.177754 & 2.246220 & -1.301720 \\
\hline 18 & 16 & 0 & 3.020619 & 3.683165 & 0.505227 \\
\hline 19 & 6 & 0 & 2.794080 & 2.138432 & -1.559013 \\
\hline 20 & 1 & 0 & 4.939138 & 1.785681 & -1.918436 \\
\hline 21 & 6 & 0 & 2.008700 & 2.853679 & -0.668700 \\
\hline 22 & 1 & 0 & 2.366801 & 1.564808 & -2.372265 \\
\hline 23 & 6 & 0 & 4.498079 & -3.054948 & -0.136178 \\
\hline 24 & 6 & 0 & 4.105838 & -2.280981 & -1.210715 \\
\hline 25 & 16 & 0 & 3.091684 & -3.671672 & 0.711696 \\
\hline 26 & 6 & 0 & 2.703619 & -2.158937 & -1.349459 \\
\hline 27 & 1 & 0 & 4.817481 & -1.837611 & -1.895998 \\
\hline 28 & 6 & 0 & 1.990399 & -2.846548 & -0.384608 \\
\hline 29 & 1 & 0 & 2.215027 & -1.586713 & -2.128524 \\
\hline 30 & 6 & 0 & -1.990468 & -2.875545 & 0.229600 \\
\hline 31 & 16 & 0 & -3.147650 & -3.548589 & -0.912418 \\
\hline 32 & 6 & 0 & -2.652964 & -2.319212 & 1.309259 \\
\hline 33 & 6 & 0 & -4.508179 & -3.048367 & 0.076629 \\
\hline 34 & 6 & 0 & -4.059390 & -2.422196 & 1.223531 \\
\hline 35 & 1 & 0 & -2.124487 & -1.853961 & 2.132289 \\
\hline 36 & 6 & 0 & -5.868467 & -3.390853 & -0.314951 \\
\hline 37 & 1 & 0 & -4.733989 & -2.067590 & 1.992609 \\
\hline 38 & 6 & 0 & -6.994947 & -2.640964 & -0.219421 \\
\hline 39 & 1 & 0 & -5.997442 & -4.393140 & -0.719592 \\
\hline 40 & 6 & 0 & -7.215788 & -1.246930 & 0.102416 \\
\hline 41 & 1 & 0 & -7.921619 & -3.153985 & -0.472741 \\
\hline 42 & 6 & 0 & -8.467298 & -0.676270 & 0.266920 \\
\hline 43 & 16 & 0 & -5.976662 & -0.004023 & 0.175782 \\
\hline 44 & 6 & 0 & -8.437351 & 0.719445 & 0.468589 \\
\hline 45 & 1 & 0 & -9.379175 & -1.262741 & 0.235447 \\
\hline 46 & 6 & 0 & -7.164219 & 1.265240 & 0.433309 \\
\hline 47 & 1 & 0 & -9.321396 & 1.324534 & 0.638128 \\
\hline
\end{tabular}




\begin{tabular}{|c|c|c|c|c|c|}
\hline 48 & 6 & 0 & -6.871653 & 2.656925 & 0.702528 \\
\hline 49 & 1 & 0 & -7.735429 & 3.184919 & 1.103875 \\
\hline 50 & 6 & 0 & -5.737038 & 3.395556 & 0.597625 \\
\hline 51 & 1 & 0 & -5.788166 & 4.400883 & 1.011974 \\
\hline 52 & 6 & 0 & -4.465588 & 3.041981 & -0.011228 \\
\hline 53 & 6 & 0 & -4.208612 & 2.348276 & -1.179451 \\
\hline 54 & 16 & 0 & -2.962286 & 3.616277 & 0.693898 \\
\hline 55 & 6 & 0 & -2.839037 & 2.251985 & -1.502692 \\
\hline 56 & 1 & 0 & -4.998198 & 1.942771 & -1.798962 \\
\hline 57 & 6 & 0 & -2.009302 & 2.883168 & -0.589055 \\
\hline 58 & 1 & 0 & -2.453202 & 1.742329 & -2.376922 \\
\hline 59 & 6 & 0 & 0.610649 & 2.919334 & -0.623875 \\
\hline 60 & 6 & 0 & -0.609860 & 2.929025 & -0.598267 \\
\hline 61 & 6 & 0 & 0.598610 & -2.894650 & -0.198816 \\
\hline 62 & 6 & 0 & -0.605769 & -2.908544 & -0.004709 \\
\hline $\begin{array}{l}\text { wor } \\
\text { met } \\
\text { ima }\end{array}$ & & & & & \\
\hline
\end{tabular}

Table S9. Cartesian coordinate of optimized structure of $\boldsymbol{2}_{\text {in }}$ at B3LYP/6-31G(d,p) level.

\begin{tabular}{|c|c|c|c|c|c|}
\hline \multirow{2}{*}{$\begin{array}{l}\text { Center } \\
\text { Number }\end{array}$} & \multirow{2}{*}{$\begin{array}{l}\text { Atomic } \\
\text { Number }\end{array}$} & \multirow{2}{*}{$\begin{array}{l}\text { Atomic } \\
\text { Type }\end{array}$} & \multicolumn{3}{|c|}{ Coordinates (Angstroms) } \\
\hline & & & $\mathrm{X}$ & $\mathrm{Y}$ & $\mathrm{Z}$ \\
\hline 1 & 6 & 0 & -0.936454 & 0.857519 & 6.598547 \\
\hline 2 & 6 & 0 & -0.523327 & 0.468412 & 7.867432 \\
\hline 3 & 6 & 0 & 0.523327 & -0.468412 & 7.867432 \\
\hline 4 & 6 & 0 & 0.936454 & -0.857519 & 6.598547 \\
\hline 5 & 16 & 0 & 0.000000 & 0.000000 & 5.388984 \\
\hline 6 & 1 & 0 & -0.980992 & 0.864724 & 8.767292 \\
\hline 7 & 1 & 0 & 0.980992 & -0.864724 & 8.767292 \\
\hline 8 & 6 & 0 & 2.041554 & -1.755418 & 6.368121 \\
\hline 9 & 1 & 0 & 2.578730 & -1.945076 & 7.296214 \\
\hline 10 & 6 & 0 & -2.041554 & 1.755418 & 6.368121 \\
\hline 11 & 1 & 0 & -2.578730 & 1.945076 & 7.296214 \\
\hline 12 & 6 & 0 & 2.582484 & -2.386827 & 5.288576 \\
\hline 13 & 1 & 0 & 3.504774 & -2.920076 & 5.513796 \\
\hline 14 & 6 & 0 & -2.582484 & 2.386827 & 5.288576 \\
\hline 15 & 1 & 0 & -3.504774 & 2.920076 & 5.513796 \\
\hline 16 & 6 & 0 & 2.234081 & -2.541876 & 3.895186 \\
\hline 17 & 6 & 0 & 3.114985 & -3.054823 & 2.949295 \\
\hline 18 & 16 & 0 & 0.672027 & -2.257787 & 3.151305 \\
\hline 19 & 6 & 0 & 2.569876 & -3.201479 & 1.660110 \\
\hline 20 & 1 & 0 & 4.136603 & -3.316113 & 3.202744 \\
\hline 21 & 6 & 0 & 1.239254 & -2.821109 & 1.584962 \\
\hline 22 & 1 & 0 & 3.114416 & -3.577180 & 0.802548 \\
\hline 23 & 6 & 0 & -2.234081 & 2.541876 & 3.895186 \\
\hline 24 & 6 & 0 & -3.114985 & 3.054823 & 2.949295 \\
\hline 25 & 16 & 0 & -0.672027 & 2.257787 & 3.151305 \\
\hline 26 & 6 & 0 & -2.569876 & 3.201479 & 1.660110 \\
\hline 27 & 1 & 0 & -4.136603 & 3.316113 & 3.202744 \\
\hline 28 & 6 & 0 & -1.239254 & 2.821109 & 1.584962 \\
\hline 29 & 1 & 0 & -3.114416 & 3.577180 & 0.802548 \\
\hline 30 & 6 & 0 & 0.385059 & -2.831328 & 0.473865 \\
\hline 31 & 6 & 0 & -0.385059 & 2.831328 & 0.473865 \\
\hline 32 & 6 & 0 & 0.385059 & 2.831328 & -0.473865 \\
\hline 33 & 6 & 0 & 1.239254 & 2.821109 & -1.584962 \\
\hline 34 & 16 & 0 & 0.672027 & 2.257787 & -3.151305 \\
\hline 35 & 6 & 0 & 2.569876 & 3.201479 & -1.660110 \\
\hline
\end{tabular}




$\begin{array}{lrrrrr}36 & 6 & 0 & 2.234081 & 2.541876 & -3.895186 \\ 37 & 6 & 0 & 3.114985 & 3.054823 & -2.949295 \\ 38 & 1 & 0 & 3.114416 & 3.577180 & -0.802548 \\ 39 & 6 & 0 & 2.582484 & 2.386827 & -5.288576 \\ 40 & 1 & 0 & 4.136603 & 3.316113 & -3.202744 \\ 41 & 6 & 0 & 2.041554 & 1.755418 & -6.368121 \\ 42 & 1 & 0 & 3.504774 & 2.920076 & -5.513796 \\ 43 & 6 & 0 & 0.936454 & 0.857519 & -6.598547 \\ 44 & 1 & 0 & 2.578730 & 1.945076 & -7.296214 \\ 45 & 6 & 0 & 0.523327 & 0.468412 & -7.867432 \\ 46 & 16 & 0 & 0.000000 & 0.000000 & -5.388984 \\ 47 & 6 & 0 & -0.523327 & -0.468412 & -7.867432 \\ 48 & 1 & 0 & 0.980992 & 0.864724 & -8.767292 \\ 49 & 6 & 0 & -0.936454 & -0.857519 & -6.598547 \\ 50 & 1 & 0 & -0.980992 & -0.864724 & -8.767292 \\ 51 & 6 & 0 & -2.041554 & -1.755418 & -6.368121 \\ 52 & 1 & 0 & -2.578730 & -1.945076 & -7.296214 \\ 53 & 6 & 0 & -2.582484 & -2.386827 & -5.288576 \\ 54 & 1 & 0 & -3.504774 & -2.920076 & -5.513796 \\ 55 & 6 & 0 & -2.234081 & -2.541876 & -3.895186 \\ 56 & 6 & 0 & -3.114985 & -3.054823 & -2.949295 \\ 57 & 16 & 0 & -0.672027 & -2.257787 & -3.151305 \\ 58 & 6 & 0 & -2.569876 & -3.201479 & -1.660110 \\ 59 & 1 & 0 & -4.136603 & -3.316113 & -3.202744 \\ 60 & 6 & 0 & -1.239254 & -2.821109 & -1.584962 \\ 61 & 1 & 0 & -3.114416 & -3.577180 & -0.802548 \\ 62 & 6 & 0 & -0.385059 & -2.831328 & -0.473865\end{array}$

Compound: $\boldsymbol{2}_{\text {in }}$ (See Table S2)

Method: B3LYP/6-31G(d,p)

Key word: opt freq scf $=$ (direct,tight)

Symmetry: $D_{2}$

\# of imaginary frequencies: 0

Energy: - 3772.8206963 Hartree

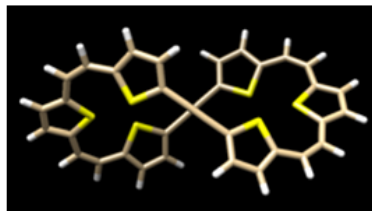

Table S10. Cartesian coordinate of optimized structure of 3-i at B3LYP/6-31G(d,p) level.

\begin{tabular}{cccccc}
$\begin{array}{c}\text { Center } \\
\text { Number }\end{array}$ & $\begin{array}{c}\text { Atomic } \\
\text { Number }\end{array}$ & $\begin{array}{c}\text { Atomic } \\
\text { Type }\end{array}$ & X & Coordinates (Angstroms) \\
\hline 1 & 6 & 0 & -7.587772 & -0.280599 & -0.151895 \\
2 & 1 & 0 & -8.634503 & -0.009386 & -0.279980 \\
3 & 6 & 0 & -7.386517 & -1.605739 & 0.088713 \\
4 & 1 & 0 & -8.306168 & -2.178651 & 0.196583 \\
5 & 6 & 0 & -6.733968 & 0.881522 & -0.261666 \\
6 & 6 & 0 & -7.160622 & 2.105301 & -0.764491 \\
7 & 16 & 0 & -5.087012 & 1.026606 & 0.316765 \\
8 & 6 & 0 & -6.193120 & 3.129387 & -0.717051 \\
9 & 1 & 0 & -8.160633 & 2.243360 & -1.161056 \\
10 & 6 & 0 & -4.996350 & 2.718884 & -0.153275 \\
11 & 1 & 0 & -6.350473 & 4.137920 & -1.079371 \\
12 & 6 & 0 & -6.227336 & -2.460938 & 0.218323 \\
13 & 6 & 0 & -6.284240 & -3.759978 & 0.710376 \\
14 & 16 & 0 & -4.597658 & -2.108684 & -0.320953 \\
15 & 6 & 0 & -5.057366 & -4.452485 & 0.684864 \\
16 & 1 & 0 & -7.207596 & -4.190077 & 1.082894 \\
17 & 6 & 0 & -4.022526 & -3.702787 & 0.148898 \\
18 & 1 & 0 & -4.918038 & -5.465498 & 1.041961 \\
19 & 6 & 0 & -2.670313 & -4.023928 & -0.026408 \\
20 & 6 & 0 & -3.796731 & 3.415702 & 0.045826 \\
21 & 6 & 0 & -1.474836 & -4.200446 & -0.203077 \\
22 & 6 & 0 & -2.694910 & 3.902959 & 0.244987 \\
& 6 & & & &
\end{tabular}




\begin{tabular}{|c|c|c|c|c|c|}
\hline 23 & 6 & 0 & -0.098007 & -4.361190 & -0.405038 \\
\hline 24 & 6 & 0 & 0.705229 & -3.684971 & -1.309638 \\
\hline 25 & 16 & 0 & 0.872171 & -5.459394 & 0.566890 \\
\hline 26 & 6 & 0 & 2.069461 & -4.036211 & -1.225630 \\
\hline 27 & 1 & 0 & 0.302858 & -2.966240 & -2.013023 \\
\hline 28 & 6 & 0 & 2.351052 & -4.977458 & -0.251882 \\
\hline 29 & 1 & 0 & 2.828332 & -3.639311 & -1.887408 \\
\hline 30 & 6 & 0 & -1.405615 & 4.403807 & 0.471595 \\
\hline 31 & 6 & 0 & -0.521344 & 4.021307 & 1.466447 \\
\hline 32 & 16 & 0 & -0.647620 & 5.575306 & -0.600265 \\
\hline 33 & 6 & 0 & 0.740863 & 4.647382 & 1.369486 \\
\hline 34 & 1 & 0 & -0.792453 & 3.309801 & 2.236797 \\
\hline 35 & 6 & 0 & 0.859447 & 5.512341 & 0.298835 \\
\hline 36 & 1 & 0 & 1.542659 & 4.492374 & 2.080479 \\
\hline 37 & 6 & 0 & 3.602261 & -5.628115 & 0.092881 \\
\hline 38 & 1 & 0 & 3.513449 & -6.682507 & 0.348074 \\
\hline 39 & 6 & 0 & 1.984610 & 6.350558 & -0.090129 \\
\hline 40 & 1 & 0 & 1.737665 & 7.378045 & -0.350974 \\
\hline 41 & 6 & 0 & 4.863104 & -5.122726 & 0.144834 \\
\hline 42 & 1 & 0 & 5.641861 & -5.855136 & 0.352516 \\
\hline 43 & 6 & 0 & 3.297048 & 6.014014 & -0.169615 \\
\hline 44 & 1 & 0 & 3.976165 & 6.832034 & -0.405762 \\
\hline 45 & 6 & 0 & 5.406698 & -3.788438 & 0.044794 \\
\hline 46 & 6 & 0 & 6.764188 & -3.506865 & 0.100122 \\
\hline 47 & 16 & 0 & 4.495017 & -2.285902 & -0.026785 \\
\hline 48 & 6 & 0 & 7.074039 & -2.131537 & 0.081903 \\
\hline 49 & 1 & 0 & 7.509859 & -4.292146 & 0.162068 \\
\hline 50 & 6 & 0 & 5.958326 & -1.312985 & 0.024333 \\
\hline 51 & 1 & 0 & 8.083819 & -1.737521 & 0.114938 \\
\hline 52 & 6 & 0 & 3.990779 & 4.749276 & -0.069510 \\
\hline 53 & 6 & 0 & 5.369642 & 4.612889 & -0.170643 \\
\hline 54 & 16 & 0 & 3.256577 & 3.162029 & 0.046040 \\
\hline 55 & 6 & 0 & 5.830893 & 3.281429 & -0.155446 \\
\hline 56 & 1 & 0 & 6.023457 & 5.473259 & -0.267705 \\
\hline 57 & 6 & 0 & 4.809896 & 2.350139 & -0.048622 \\
\hline 58 & 1 & 0 & 6.877625 & 3.010211 & -0.229505 \\
\hline 59 & 6 & 0 & 5.944313 & 0.125001 & -0.003281 \\
\hline 60 & 1 & 0 & 6.929360 & 0.587905 & -0.020794 \\
\hline 61 & 6 & 0 & 4.835865 & 0.910112 & -0.003775 \\
\hline 62 & 1 & 0 & 3.862269 & 0.424488 & 0.035328 \\
\hline . & $s c f=$ & & & & \\
\hline
\end{tabular}

Table S11. Cartesian coordinate of optimized structure of 3-ii at B3LYP/6-31G(d,p) level.

\begin{tabular}{cccccc}
$\begin{array}{c}\text { Center } \\
\text { Number }\end{array}$ & $\begin{array}{c}\text { Atomic } \\
\text { Number }\end{array}$ & $\begin{array}{c}\text { Atomic } \\
\text { Type }\end{array}$ & \multicolumn{2}{c}{ Coordinates (Angstroms) } \\
- \\
1 & 6 & 0 & -7.524202 & -0.341947 & -0.401201 \\
2 & 1 & 0 & -8.570001 & -0.081840 & -0.557201 \\
3 & 6 & 0 & -7.301611 & -1.688348 & -0.405201 \\
4 & 1 & 0 & -8.208714 & -2.269743 & -0.563001 \\
5 & 6 & 0 & -6.729795 & 0.855148 & -0.271101 \\
6 & 6 & 0 & -7.260487 & 2.133352 & -0.423601 \\
7 & 16 & 0 & -5.018094 & 0.975038 & 0.079599
\end{tabular}




\begin{tabular}{|c|c|c|c|c|c|}
\hline 8 & 6 & 0 & -6.329181 & 3.178846 & -0.273201 \\
\hline 9 & 1 & 0 & -8.309986 & 2.292658 & -0.645701 \\
\hline 10 & 6 & 0 & -5.048884 & 2.730738 & 0.007899 \\
\hline 11 & 1 & 0 & -6.569974 & 4.231147 & -0.363001 \\
\hline 12 & 6 & 0 & -6.165616 & -2.569055 & -0.282301 \\
\hline 13 & 6 & 0 & -6.263624 & -3.949255 & -0.438701 \\
\hline 14 & 16 & 0 & -4.501713 & -2.140465 & 0.061299 \\
\hline 15 & 6 & 0 & -5.048729 & -4.645662 & -0.296201 \\
\hline 16 & 1 & 0 & -7.209427 & -4.432549 & -0.657601 \\
\hline 17 & 6 & 0 & -3.974924 & -3.814868 & -0.017501 \\
\hline 18 & 1 & 0 & -4.943735 & -5.719663 & -0.389401 \\
\hline 19 & 6 & 0 & -2.620025 & -4.115777 & 0.176399 \\
\hline 20 & 6 & 0 & -3.854579 & 3.437831 & 0.207999 \\
\hline 21 & 6 & 0 & -1.419126 & -4.260784 & 0.344799 \\
\hline 22 & 6 & 0 & -2.746776 & 3.921624 & 0.380699 \\
\hline 23 & 6 & 0 & -0.035727 & -4.388393 & 0.529499 \\
\hline 24 & 6 & 0 & 0.775777 & -3.642198 & 1.368499 \\
\hline 25 & 16 & 0 & 0.932666 & -5.526898 & -0.397301 \\
\hline 26 & 6 & 0 & 2.145075 & -3.971606 & 1.270699 \\
\hline 27 & 1 & 0 & 0.376282 & -2.888095 & 2.035699 \\
\hline 28 & 6 & 0 & 2.420969 & -4.966408 & 0.350299 \\
\hline 29 & 1 & 0 & 2.911978 & -3.520311 & 1.886799 \\
\hline 30 & 6 & 0 & -1.451173 & 4.424616 & 0.569699 \\
\hline 31 & 6 & 0 & -0.525476 & 4.025510 & 1.518399 \\
\hline 32 & 16 & 0 & -0.739366 & 5.616812 & -0.511401 \\
\hline 33 & 6 & 0 & 0.731728 & 4.654903 & 1.379999 \\
\hline 34 & 1 & 0 & -0.763380 & 3.299112 & 2.285899 \\
\hline 35 & 6 & 0 & 0.803734 & 5.539702 & 0.321599 \\
\hline 36 & 1 & 0 & 1.562827 & 4.488598 & 2.053799 \\
\hline 37 & 6 & 0 & 3.675865 & -5.611215 & 0.007199 \\
\hline 38 & 1 & 0 & 3.603459 & -6.681815 & -0.175301 \\
\hline 39 & 6 & 0 & 1.909839 & 6.386796 & -0.101901 \\
\hline 40 & 1 & 0 & 1.649645 & 7.418197 & -0.332601 \\
\hline 41 & 6 & 0 & 4.920969 & -5.083223 & -0.129501 \\
\hline 42 & 1 & 0 & 5.706764 & -5.811428 & -0.325401 \\
\hline 43 & 6 & 0 & 3.217237 & 6.054788 & -0.251201 \\
\hline 44 & 1 & 0 & 3.881442 & 6.878784 & -0.508201 \\
\hline 45 & 6 & 0 & 5.439377 & -3.735526 & -0.139501 \\
\hline 46 & 6 & 0 & 6.783179 & -3.434934 & -0.311401 \\
\hline 47 & 16 & 0 & 4.505386 & -2.246220 & -0.076801 \\
\hline 48 & 6 & 0 & 7.065287 & -2.056136 & -0.389301 \\
\hline 49 & 1 & 0 & 7.537774 & -4.210139 & -0.389501 \\
\hline 50 & 6 & 0 & 5.941192 & -1.253529 & -0.284801 \\
\hline 51 & 1 & 0 & 8.061390 & -1.647942 & -0.519801 \\
\hline 52 & 6 & 0 & 3.920029 & 4.792083 & -0.206701 \\
\hline 53 & 6 & 0 & 5.290628 & 4.664975 & -0.395401 \\
\hline 54 & 16 & 0 & 3.204019 & 3.199288 & -0.056701 \\
\hline 55 & 6 & 0 & 5.759920 & 3.336772 & -0.418401 \\
\hline 56 & 1 & 0 & 5.932033 & 5.530271 & -0.526301 \\
\hline 57 & 6 & 0 & 4.753414 & 2.397978 & -0.253701 \\
\hline 58 & 1 & 0 & 6.802018 & 3.073166 & -0.556301 \\
\hline 59 & 6 & 0 & 5.904501 & 0.183571 & -0.324301 \\
\hline 60 & 1 & 0 & 6.876504 & 0.656765 & -0.451701 \\
\hline 61 & 6 & 0 & 4.793706 & 0.957978 & -0.216801 \\
\hline 62 & 1 & 0 & 3.831803 & 0.463884 & -0.091001 \\
\hline
\end{tabular}

Compound: 3-ii (See Table S3)

Method: B3LYP/6-31G(d,p)

Key word: opt freq scf=(direct,tight)

Symmetry: $C_{1}$

\# of imaginary frequencies: 0

Energy: -3772.8318911 Hartree

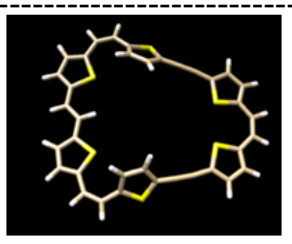


Table S12. Cartesian coordinate of optimized structure of 4-i at B3LYP/6-31G(d,p) level.

\begin{tabular}{|c|c|c|c|c|c|}
\hline \multirow{2}{*}{$\begin{array}{l}\text { Center } \\
\text { Number }\end{array}$} & \multirow{2}{*}{$\begin{array}{l}\text { Atomic } \\
\text { Number }\end{array}$} & \multirow{2}{*}{$\begin{array}{l}\text { Atomic } \\
\text { Type }\end{array}$} & \multicolumn{3}{|c|}{ Coordinates (Angstroms) } \\
\hline & & & $\mathrm{X}$ & $\mathrm{Y}$ & Z \\
\hline 1 & 6 & 0 & 6.208700 & -4.748600 & 0.000000 \\
\hline 2 & 1 & 0 & 7.200700 & -5.197300 & 0.000000 \\
\hline 3 & 6 & 0 & 5.203400 & -5.673400 & 0.000000 \\
\hline 4 & 1 & 0 & 5.570200 & -6.698700 & 0.000000 \\
\hline 5 & 6 & 0 & 3.763300 & -5.665600 & 0.000000 \\
\hline 6 & 6 & 0 & 2.999200 & -6.831800 & 0.000000 \\
\hline 7 & 1 & 0 & 3.460100 & -7.813800 & 0.000000 \\
\hline 8 & 6 & 0 & 1.608600 & -6.625000 & 0.000000 \\
\hline 9 & 1 & 0 & 0.883300 & -7.430400 & 0.000000 \\
\hline 10 & 6 & 0 & 1.252000 & -5.283800 & 0.000000 \\
\hline 11 & 16 & 0 & 2.689300 & -4.282800 & 0.000000 \\
\hline 12 & 6 & 0 & -2.510300 & -4.522600 & 0.000000 \\
\hline 13 & 6 & 0 & -3.780300 & -5.080000 & 0.000000 \\
\hline 14 & 1 & 0 & -3.939800 & -6.152800 & 0.000000 \\
\hline 15 & 6 & 0 & -4.828800 & -4.134500 & 0.000000 \\
\hline 16 & 1 & 0 & -5.881500 & -4.389500 & 0.000000 \\
\hline 17 & 6 & 0 & -4.383200 & -2.822200 & 0.000000 \\
\hline 18 & 16 & 0 & -2.621500 & -2.770700 & 0.000000 \\
\hline 19 & 6 & 0 & -5.097100 & -1.619200 & 0.000000 \\
\hline 20 & 6 & 0 & -5.632500 & -0.520600 & 0.000000 \\
\hline 21 & 6 & 0 & -6.141500 & 0.782500 & 0.000000 \\
\hline 22 & 6 & 0 & -7.450100 & 1.242400 & 0.000000 \\
\hline 23 & 1 & 0 & -8.301900 & 0.573400 & 0.000000 \\
\hline 24 & 6 & 0 & -7.541200 & 2.646300 & 0.000000 \\
\hline 25 & 1 & 0 & -8.482100 & 3.185500 & 0.000000 \\
\hline 26 & 6 & 0 & -6.316900 & 3.311300 & 0.000000 \\
\hline 27 & 6 & 0 & -6.208700 & 4.748600 & 0.000000 \\
\hline 28 & 1 & 0 & -7.200700 & 5.197300 & 0.000000 \\
\hline 29 & 6 & 0 & -5.203400 & 5.673400 & 0.000000 \\
\hline 30 & 1 & 0 & -5.570200 & 6.698700 & 0.000000 \\
\hline 31 & 6 & 0 & -3.763300 & 5.665600 & 0.000000 \\
\hline 32 & 6 & 0 & -2.999200 & 6.831800 & 0.000000 \\
\hline 33 & 1 & 0 & -3.460100 & 7.813800 & 0.000000 \\
\hline 34 & 6 & 0 & -1.608600 & 6.625000 & 0.000000 \\
\hline 35 & 1 & 0 & -0.883300 & 7.430400 & 0.000000 \\
\hline 36 & 6 & 0 & -1.252000 & 5.283800 & 0.000000 \\
\hline 37 & 16 & 0 & -5.017900 & 2.134300 & 0.000000 \\
\hline 38 & 16 & 0 & -2.689300 & 4.282800 & 0.000000 \\
\hline 39 & 6 & 0 & 2.510300 & 4.522600 & 0.000000 \\
\hline 40 & 6 & 0 & 3.780300 & 5.080000 & 0.000000 \\
\hline 41 & 1 & 0 & 3.939800 & 6.152800 & 0.000000 \\
\hline 42 & 6 & 0 & 4.828800 & 4.134500 & 0.000000 \\
\hline 43 & 1 & 0 & 5.881500 & 4.389500 & 0.000000 \\
\hline 44 & 6 & 0 & 4.383200 & 2.822200 & 0.000000 \\
\hline 45 & 16 & 0 & 2.621500 & 2.770700 & 0.000000 \\
\hline 46 & 6 & 0 & 5.097100 & 1.619200 & 0.000000 \\
\hline 47 & 6 & 0 & 5.632500 & 0.520600 & 0.000000 \\
\hline 48 & 6 & 0 & 6.141500 & -0.782500 & 0.000000 \\
\hline 49 & 6 & 0 & 7.450100 & -1.242400 & 0.000000 \\
\hline 50 & 1 & 0 & 8.301900 & -0.573400 & 0.000000 \\
\hline 51 & 6 & 0 & 7.541200 & -2.646300 & 0.000000 \\
\hline 52 & 1 & 0 & 8.482100 & -3.185500 & 0.000000 \\
\hline 53 & 6 & 0 & 6.316900 & -3.311300 & 0.000000 \\
\hline 54 & 16 & 0 & 5.017900 & -2.134300 & 0.000000 \\
\hline 55 & 6 & 0 & 1.252000 & 5.221100 & 0.000000 \\
\hline 56 & 1 & 0 & 1.328800 & 6.306700 & 0.000000 \\
\hline 57 & 6 & 0 & -1.252000 & -5.221100 & 0.000000 \\
\hline 58 & 1 & 0 & -1.328800 & -6.306700 & 0.000000 \\
\hline 59 & 6 & 0 & 0.029600 & 4.628500 & 0.000000 \\
\hline
\end{tabular}




$\begin{array}{lllrrr}60 & 1 & 0 & -0.008000 & 3.540400 & 0.000000 \\ 61 & 6 & 0 & -0.029600 & -4.628500 & 0.000000 \\ 62 & 1 & 0 & 0.008000 & -3.540400 & 0.000000\end{array}$

Compound: 4-i (See Table S4)

Method: B3LYP/6-31G(d,p)

Key word: opt freq scf=(direct, tight)

Symmetry: $C_{2 \mathrm{~h}}$

\# of imaginary frequencies: 0

Energy: -3772.8406298 Hartree

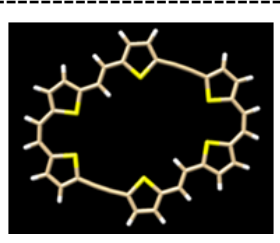

Table S13. Cartesian coordinate of optimized structure of 4-ii at B3LYP/6-31G(d,p) level.

\begin{tabular}{|c|c|c|c|c|c|}
\hline \multirow{2}{*}{$\begin{array}{l}\text { Center } \\
\text { Number }\end{array}$} & \multirow{2}{*}{$\begin{array}{l}\text { Atomic } \\
\text { Number }\end{array}$} & \multirow{2}{*}{$\begin{array}{l}\text { Atomic } \\
\text { Type }\end{array}$} & \multicolumn{3}{|c|}{ Coordinates (Angstroms) } \\
\hline & & & $\mathrm{X}$ & $\mathrm{Y}$ & $\mathrm{Z}$ \\
\hline 1 & 6 & 0 & -0.950011 & 4.639558 & -0.000000 \\
\hline 2 & 16 & 0 & -0.000000 & 3.160685 & -0.000000 \\
\hline 3 & 6 & 0 & -0.111148 & 5.745470 & -0.000000 \\
\hline 4 & 1 & 0 & -0.491445 & 6.759886 & -0.000000 \\
\hline 5 & 6 & 0 & 1.259559 & 5.413939 & 0.000000 \\
\hline 6 & 1 & 0 & 2.048521 & 6.157152 & 0.000000 \\
\hline 7 & 6 & 0 & 1.510284 & 4.048116 & 0.000000 \\
\hline 8 & 6 & 0 & 2.744818 & 3.304125 & 0.000000 \\
\hline 9 & 1 & 0 & 2.639423 & 2.220652 & 0.000000 \\
\hline 10 & 6 & 0 & 3.998424 & 3.826242 & 0.000000 \\
\hline 11 & 1 & 0 & 4.128334 & 4.906998 & 0.000000 \\
\hline 12 & 6 & 0 & 5.227402 & 3.080490 & 0.000000 \\
\hline 13 & 6 & 0 & 6.511573 & 3.603096 & 0.000000 \\
\hline 14 & 1 & 0 & 6.702357 & 4.670660 & 0.000000 \\
\hline 15 & 16 & 0 & 5.293848 & 1.325392 & 0.000000 \\
\hline 16 & 6 & 0 & 7.520874 & 2.624031 & 0.000000 \\
\hline 17 & 1 & 0 & 8.581507 & 2.850148 & 0.000000 \\
\hline 18 & 6 & 0 & 7.051686 & 1.314425 & 0.000000 \\
\hline 19 & 6 & 0 & 7.934089 & 0.177376 & 0.000000 \\
\hline 20 & 1 & 0 & 8.969219 & 0.515471 & 0.000000 \\
\hline 21 & 6 & 0 & 7.823951 & -1.183602 & 0.000000 \\
\hline 22 & 1 & 0 & 8.790793 & -1.684399 & 0.000000 \\
\hline 23 & 6 & 0 & 6.768815 & -2.164575 & 0.000000 \\
\hline 24 & 16 & 0 & 5.033979 & -1.900968 & 0.000000 \\
\hline 25 & 6 & 0 & 7.029002 & -3.532887 & 0.000000 \\
\hline 26 & 1 & 0 & 8.042337 & -3.919123 & 0.000000 \\
\hline 27 & 6 & 0 & 5.885838 & -4.349955 & 0.000000 \\
\hline 28 & 1 & 0 & 5.905597 & -5.432845 & 0.000000 \\
\hline 29 & 6 & 0 & 4.700472 & -3.628925 & 0.000000 \\
\hline 30 & 6 & 0 & 3.382786 & -4.098271 & 0.000000 \\
\hline 31 & 6 & 0 & 2.222491 & -4.481685 & 0.000000 \\
\hline 32 & 6 & 0 & 0.872464 & -4.848466 & 0.000000 \\
\hline 33 & 16 & 0 & -0.368684 & -3.598846 & -0.000000 \\
\hline 34 & 6 & 0 & 0.291524 & -6.107158 & 0.000000 \\
\hline 35 & 1 & 0 & 0.879739 & -7.016659 & 0.000000 \\
\hline 36 & 6 & 0 & -1.119826 & -6.071550 & -0.000000 \\
\hline 37 & 1 & 0 & -1.742487 & -6.959476 & -0.000000 \\
\hline 38 & 6 & 0 & -1.656759 & -4.793141 & -0.000000 \\
\hline 39 & 6 & 0 & -3.049631 & -4.427938 & -0.000000 \\
\hline 40 & 1 & 0 & -3.749196 & -5.261582 & -0.000000 \\
\hline 41 & 6 & 0 & -3.508552 & -3.149971 & -0.000000 \\
\hline 42 & 1 & 0 & -2.767773 & -2.351735 & -0.000000 \\
\hline 43 & 6 & 0 & -4.874392 & -2.697148 & -0.000000 \\
\hline 44 & 16 & 0 & -5.139633 & -0.968233 & -0.000000 \\
\hline 45 & 6 & 0 & -6.092210 & -3.363050 & -0.000000 \\
\hline 46 & 1 & 0 & -6.177111 & -4.443757 & -0.000000 \\
\hline 47 & 6 & 0 & -7.200363 & -2.495682 & -0.000000 \\
\hline
\end{tabular}




$\begin{array}{lrrrrr}48 & 1 & 0 & -8.229792 & -2.837976 & -0.000000 \\ 49 & 6 & 0 & -6.880560 & -1.137907 & -0.000000 \\ 50 & 6 & 0 & -7.869343 & -0.089714 & -0.000000 \\ 51 & 1 & 0 & -8.870629 & -0.517703 & -0.000000 \\ 52 & 6 & 0 & -7.870689 & 1.277147 & -0.000000 \\ 53 & 1 & 0 & -8.871882 & 1.705091 & -0.000000 \\ 54 & 6 & 0 & -6.879965 & 2.324817 & -0.000000 \\ 55 & 16 & 0 & -5.138149 & 2.142406 & -0.000000 \\ 56 & 6 & 0 & -7.198049 & 3.681608 & -0.000000 \\ 57 & 1 & 0 & -8.226512 & 4.026014 & -0.000000 \\ 58 & 6 & 0 & -6.088529 & 4.549610 & -0.000000 \\ 59 & 1 & 0 & -6.158147 & 5.630560 & -0.000000 \\ 60 & 6 & 0 & -4.875305 & 3.878202 & -0.000000 \\ 61 & 6 & 0 & -3.548679 & 4.325310 & -0.000000 \\ 62 & 6 & 0 & -2.346148 & 4.544004 & -0.000000\end{array}$

Compound: 4-ii (See Table S4)

Method: B3LYP/6-31G(d,p)

Key word: opt freq scf=(direct, tight)

Symmetry: $C_{\mathrm{s}}$

\# of imaginary frequencies: 0

Energy: -3772.8392229 Hartree

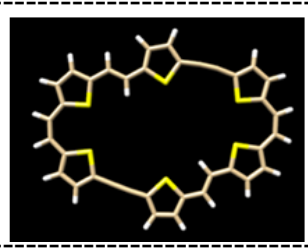

Table S14. Cartesian coordinate of optimized structure of 4-iii at B3LYP/6-31G(d,p) level.

\begin{tabular}{|c|c|c|c|c|c|}
\hline \multirow{2}{*}{$\begin{array}{c}\text { Center } \\
\text { Number }\end{array}$} & \multirow{2}{*}{$\begin{array}{l}\text { Atomic } \\
\text { Number }\end{array}$} & \multirow{2}{*}{$\begin{array}{l}\text { Atomic } \\
\text { Type }\end{array}$} & \multicolumn{3}{|c|}{ Coordinates (Angstroms) } \\
\hline & & & $\mathrm{X}$ & $\mathrm{Y}$ & $\mathrm{Z}$ \\
\hline 1 & 6 & 0 & -0.942000 & 8.004600 & 0.000000 \\
\hline 2 & 1 & 0 & -1.429100 & 8.978400 & 0.000000 \\
\hline 3 & 6 & 0 & 0.421500 & 8.090800 & 0.000000 \\
\hline 4 & 1 & 0 & 0.781600 & 9.118500 & 0.000000 \\
\hline 5 & 6 & 0 & 1.535800 & 7.178900 & 0.000000 \\
\hline 6 & 6 & 0 & 2.862000 & 7.600000 & 0.000000 \\
\hline 7 & 1 & 0 & 3.127700 & 8.651400 & 0.000000 \\
\hline 8 & 6 & 0 & 3.804500 & 6.554300 & 0.000000 \\
\hline 9 & 1 & 0 & 4.878200 & 6.707000 & 0.000000 \\
\hline 10 & 6 & 0 & 3.234300 & 5.290500 & 0.000000 \\
\hline 11 & 16 & 0 & 1.485400 & 5.423700 & 0.000000 \\
\hline 12 & 6 & 0 & 3.940900 & 1.516500 & 0.000000 \\
\hline 13 & 6 & 0 & 5.279300 & 1.147400 & 0.000000 \\
\hline 14 & 1 & 0 & 6.088500 & 1.868600 & 0.000000 \\
\hline 15 & 6 & 0 & 5.488200 & -0.247000 & 0.000000 \\
\hline 16 & 1 & 0 & 6.464900 & -0.715800 & 0.000000 \\
\hline 17 & 6 & 0 & 4.312200 & -0.985700 & 0.000000 \\
\hline 18 & 16 & 0 & 2.923500 & 0.089500 & 0.000000 \\
\hline 19 & 6 & 0 & 4.109200 & -2.370100 & 0.000000 \\
\hline 20 & 6 & 0 & 3.832200 & -3.560300 & 0.000000 \\
\hline 21 & 6 & 0 & 3.401600 & -4.891600 & 0.000000 \\
\hline 22 & 6 & 0 & 4.122400 & -6.076600 & 0.000000 \\
\hline 23 & 1 & 0 & 5.205300 & -6.099400 & 0.000000 \\
\hline 24 & 6 & 0 & 3.302600 & -7.220000 & 0.000000 \\
\hline 25 & 1 & 0 & 3.688800 & -8.233500 & 0.000000 \\
\hline 26 & 6 & 0 & 1.934100 & -6.959000 & 0.000000 \\
\hline 27 & 6 & 0 & 0.942000 & -8.004600 & 0.000000 \\
\hline 28 & 1 & 0 & 1.429100 & -8.978400 & 0.000000 \\
\hline 29 & 6 & 0 & -0.421500 & -8.090800 & 0.000000 \\
\hline 30 & 1 & 0 & -0.781600 & -9.118500 & 0.000000 \\
\hline 31 & 6 & 0 & -1.535800 & -7.178900 & 0.000000 \\
\hline 32 & 6 & 0 & -2.862000 & -7.600000 & 0.000000 \\
\hline 33 & 1 & 0 & -3.127700 & -8.651400 & 0.000000 \\
\hline 34 & 6 & 0 & -3.804500 & -6.554300 & 0.000000 \\
\hline 35 & 1 & 0 & -4.878200 & -6.707000 & 0.000000 \\
\hline
\end{tabular}




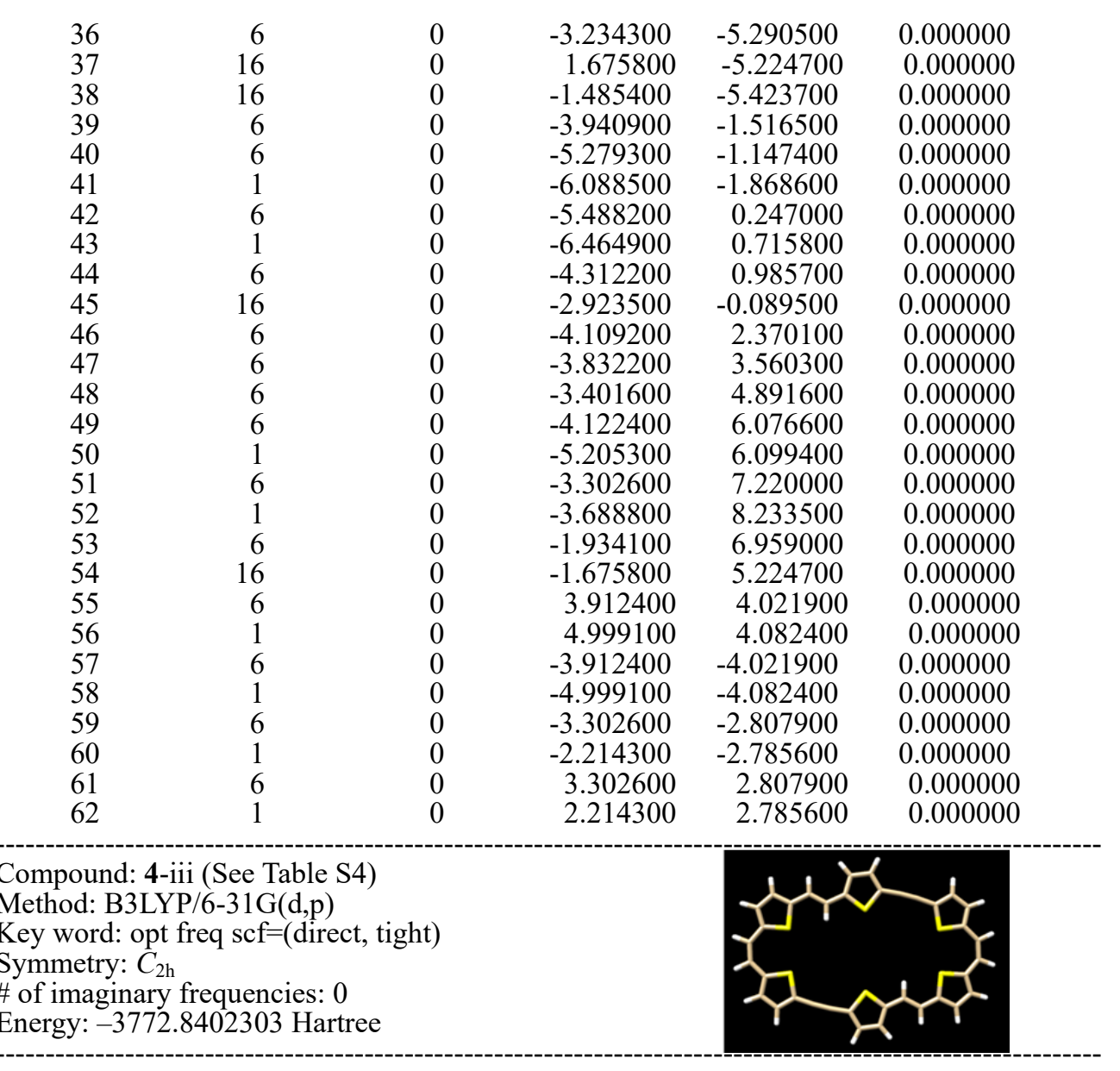

S3-5. Relative energy of 2, E,Z,Z,Z-intermediate, and 4.

It is expected that photoirradiation of 2 first generates an $E, Z, Z, Z$-intermediate, and the further photoirradiation produces 4 (relative energy: $2+48.8 \mathrm{~kJ} / \mathrm{mol}, E, Z, Z, Z$-intermediate $+30.6 \mathrm{~kJ} / \mathrm{mol}$, and $40 \mathrm{kJmol}^{-1}$ at B3LYP/6-31G(d,p) level).

Figure S5. Relative energy of 2, E,Z,Z,Z-intermediate, and 4.

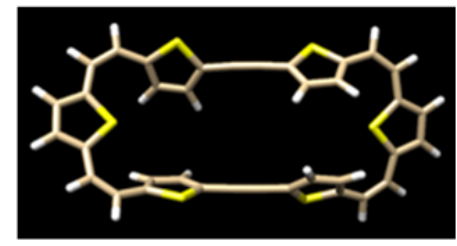

2-out_i

(close to $\mathrm{X}$-ray structure)

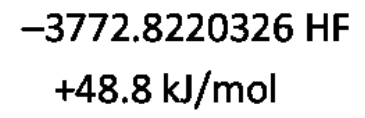

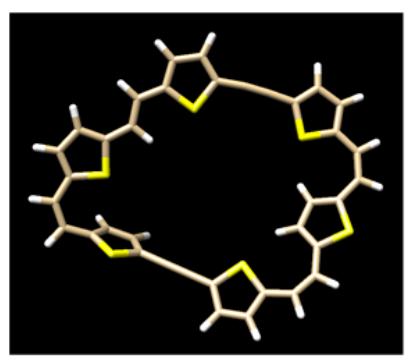

EZZZ

$$
\begin{array}{r}
-3772.828973 \mathrm{HF} \\
+30.6 \mathrm{~kJ} / \mathrm{mol}
\end{array}
$$

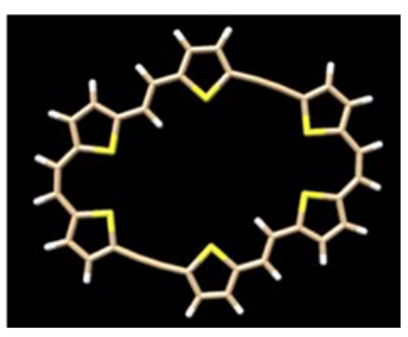

4-i

$-3772.8406298 \mathrm{HF}$

$0.00 \mathrm{~kJ} / \mathrm{mol}$ 
Table S15. Cartesian coordinate of optimized structure of E,Z,Z,Z-intermediate at B3LYP/6-31G(d,p) level.

\begin{tabular}{|c|c|c|c|c|c|}
\hline \multirow{2}{*}{$\begin{array}{l}\text { Center } \\
\text { Number }\end{array}$} & \multirow{2}{*}{$\begin{array}{l}\text { Atomic } \\
\text { Number }\end{array}$} & \multirow{2}{*}{$\begin{array}{l}\text { Atomic } \\
\text { Type }\end{array}$} & \multicolumn{3}{|c|}{ Coordinates (Angstroms) } \\
\hline & & & $\mathrm{X}$ & $\mathrm{Y}$ & $\mathrm{Z}$ \\
\hline 1 & 6 & 0 & -0.019129 & -4.772675 & 0.134294 \\
\hline 2 & 6 & 0 & 0.289867 & -6.056292 & 0.552091 \\
\hline 3 & 6 & 0 & 1.663768 & -6.251857 & 0.780240 \\
\hline 4 & 6 & 0 & 2.456353 & -5.133181 & 0.546307 \\
\hline 5 & 16 & 0 & 1.443207 & -3.794205 & 0.038452 \\
\hline 6 & 6 & 0 & -1.281654 & -4.244997 & -0.175628 \\
\hline 7 & 6 & 0 & -2.379358 & -3.789073 & -0.452759 \\
\hline 8 & 6 & 0 & -3.645588 & -3.250748 & -0.739044 \\
\hline 9 & 6 & 0 & 5.066776 & -2.908637 & 0.189685 \\
\hline 10 & 16 & 0 & -4.936718 & -3.254352 & 0.458877 \\
\hline 11 & 6 & 0 & -6.014792 & -2.383169 & -0.612876 \\
\hline 12 & 6 & 0 & -5.398079 & -2.140652 & -1.820588 \\
\hline 13 & 6 & 0 & -4.068141 & -2.623328 & -1.895648 \\
\hline 14 & 6 & 0 & 4.269420 & -2.016325 & -0.511308 \\
\hline 15 & 6 & 0 & 4.881416 & -0.766712 & -0.760865 \\
\hline 16 & 6 & 0 & 6.169738 & -0.653601 & -0.261436 \\
\hline 17 & 16 & 0 & 6.617702 & -2.151953 & 0.531527 \\
\hline 18 & 6 & 0 & -2.339832 & 4.819526 & 0.418705 \\
\hline 19 & 6 & 0 & -1.945390 & 6.142698 & 0.544314 \\
\hline 20 & 6 & 0 & -0.549428 & 6.341997 & 0.453775 \\
\hline 21 & 6 & 0 & 0.159321 & 5.167278 & 0.258947 \\
\hline 22 & 16 & 0 & -0.935978 & 3.791474 & 0.189759 \\
\hline 23 & 6 & 0 & 1.530860 & 4.932774 & 0.117152 \\
\hline 24 & 6 & 0 & 2.699263 & 4.607095 & -0.029982 \\
\hline 25 & 6 & 0 & -5.248067 & 2.318645 & 0.286292 \\
\hline 26 & 6 & 0 & 4.014428 & 4.162581 & -0.197363 \\
\hline 27 & 16 & 0 & -5.266345 & 0.603740 & -0.070409 \\
\hline 28 & 6 & 0 & -6.997689 & 0.499821 & 0.146492 \\
\hline 29 & 6 & 0 & -7.504353 & 1.751686 & 0.467823 \\
\hline 30 & 6 & 0 & -6.532045 & 2.772478 & 0.544406 \\
\hline 31 & 6 & 0 & 5.171760 & 4.890498 & -0.425183 \\
\hline 32 & 6 & 0 & 6.314345 & 4.080158 & -0.560184 \\
\hline 33 & 6 & 0 & 6.075447 & 2.715240 & -0.443652 \\
\hline 34 & 16 & 0 & 4.369059 & 2.437160 & -0.150307 \\
\hline 35 & 6 & 0 & 7.184079 & 0.375619 & -0.358842 \\
\hline 36 & 6 & 0 & 7.127155 & 1.730347 & -0.479470 \\
\hline 37 & 6 & 0 & -7.766001 & -0.725723 & 0.085790 \\
\hline
\end{tabular}




\begin{tabular}{llrrrr}
38 & 6 & 0 & -7.367057 & -1.988770 & -0.188307 \\
39 & 6 & 0 & 3.882697 & -5.151178 & 0.752743 \\
40 & 6 & 0 & 4.920524 & -4.274805 & 0.636178 \\
41 & 6 & 0 & -3.965416 & 2.971103 & 0.258985 \\
42 & 6 & 0 & -3.676180 & 4.282614 & 0.454458 \\
43 & 1 & 0 & -0.467953 & -6.819593 & 0.679215 \\
44 & 1 & 0 & 2.089890 & -7.192506 & 1.111084 \\
45 & 1 & 0 & -5.895760 & -1.615253 & -2.627151 \\
46 & 1 & 0 & -3.427022 & -2.514515 & -2.761944 \\
47 & 1 & 0 & 3.286453 & -2.266046 & -0.885887 \\
48 & 1 & 0 & 4.408221 & 0.010685 & -1.346519 \\
49 & 1 & 0 & -2.657279 & 6.946789 & 0.695318 \\
50 & 1 & 0 & -0.064378 & 7.307650 & 0.527834 \\
51 & 1 & 0 & -8.560718 & 1.913707 & 0.655217 \\
52 & 1 & 0 & -6.762109 & 3.803165 & 0.788773 \\
53 & 1 & 0 & 5.174734 & 5.971442 & -0.492532 \\
54 & 1 & 0 & 7.310696 & 4.471910 & -0.733325 \\
55 & 1 & 0 & 8.198355 & -0.017605 & -0.318162 \\
56 & 1 & 0 & 8.100440 & 2.207453 & -0.583611 \\
57 & 1 & 0 & -8.815500 & -0.586858 & 0.341638 \\
58 & 1 & 0 & -8.104907 & -2.782679 & -0.087011 \\
59 & 1 & 0 & 4.191004 & -6.130235 & 1.116344 \\
60 & 1 & 0 & 5.866652 & -4.704895 & 0.959733 \\
61 & 1 & 0 & -3.130072 & 2.303339 & 0.053155 \\
62 & 1 & 0 & -4.469683 & 5.001621 & 0.648157 \\
\hline------------------------------------------------------------------------------------
\end{tabular}

Compound: EZZZ-intermediate Method: B3LYP/6-31G(d,p) Key word: opt freq scf $=($ direct,tight $)$ Symmetry: $C_{1}$ \# of imaginary frequencies: 0 Energy: -3772.828973 Hartree

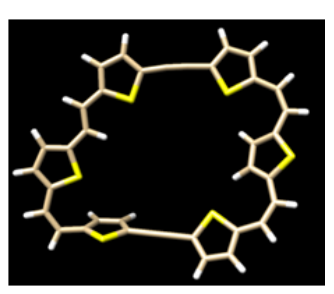

\section{S4. Comparison of total energy and HOMO-LUMO energy level}

Table S16 shows comparison of total energy in each isomer 2, 3, and 4. The most stable geometries and their HOMO-LUMO energy levels are listed. 
Table S16. Geometry, total energy, and MOs of 2-4.

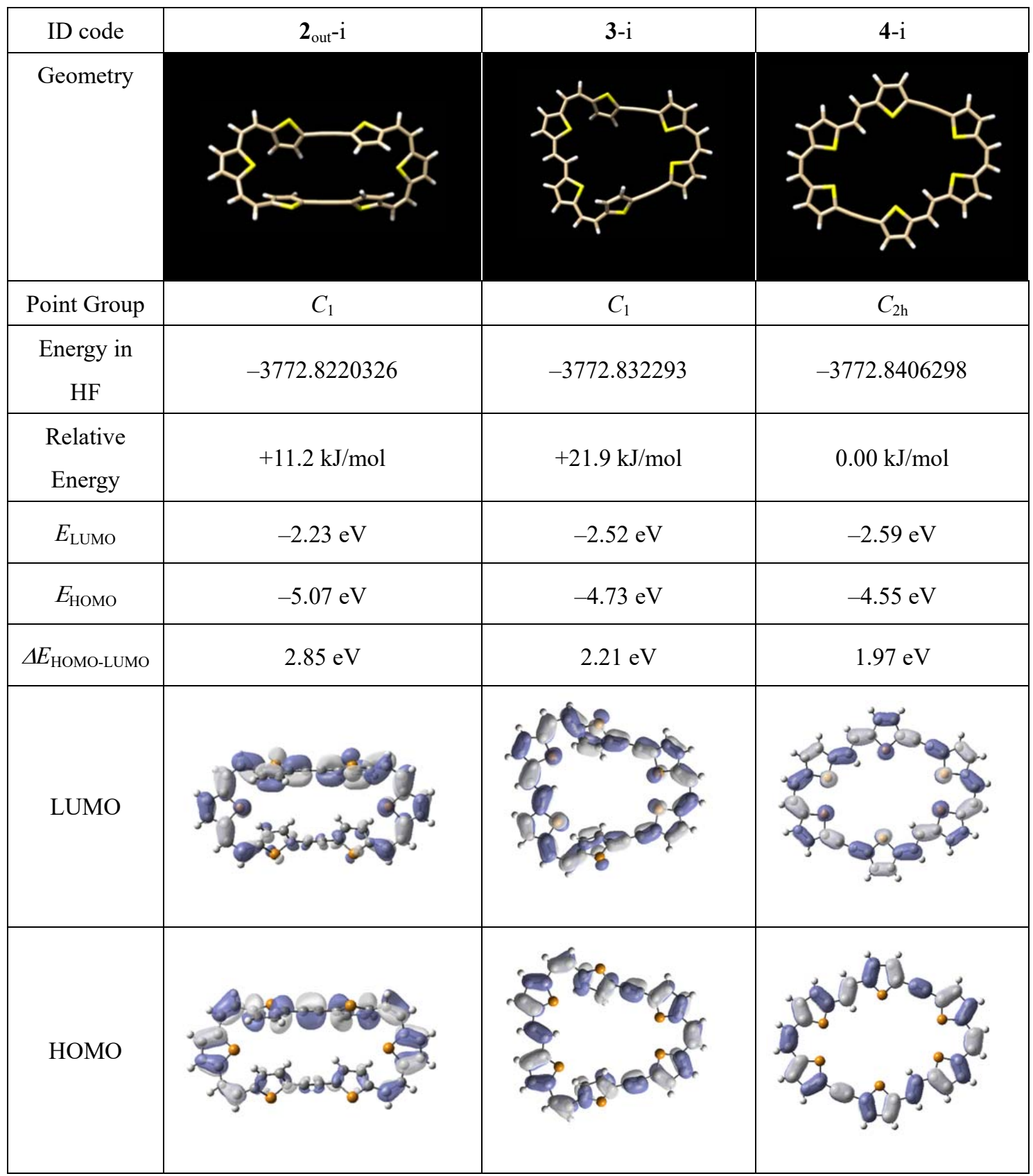




\section{S5. PXRD patterns of films of 2,3 , and 4}

Figure S6. PXRD patterns of the 2 film measured on a glass plate. (a) Before annealing. (b) After annealing at $100^{\circ} \mathrm{C}$ for $1 \mathrm{~h}$.

(a)

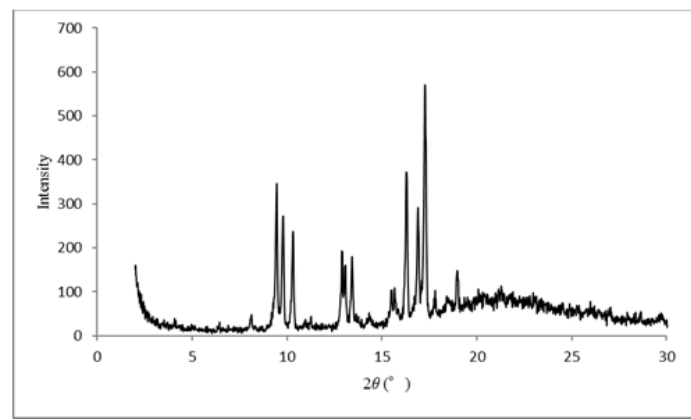

(b)

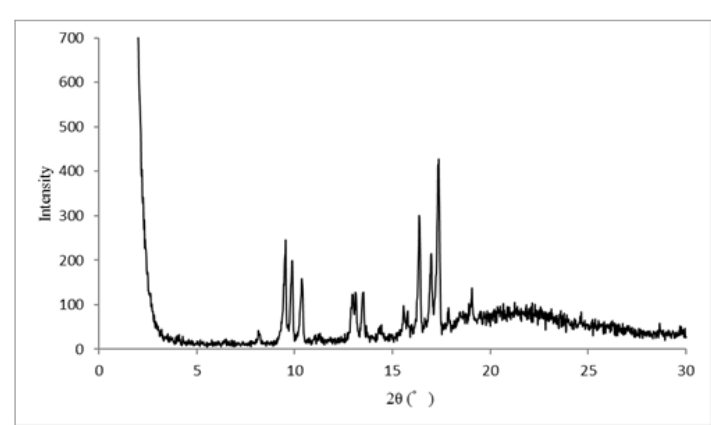

Figure S7. PXRD patterns of the 3 film measured on a glass plate. (a) Before annealing. (b) After annealing at $100{ }^{\circ} \mathrm{C}$ for $1 \mathrm{~h}$.

(a)

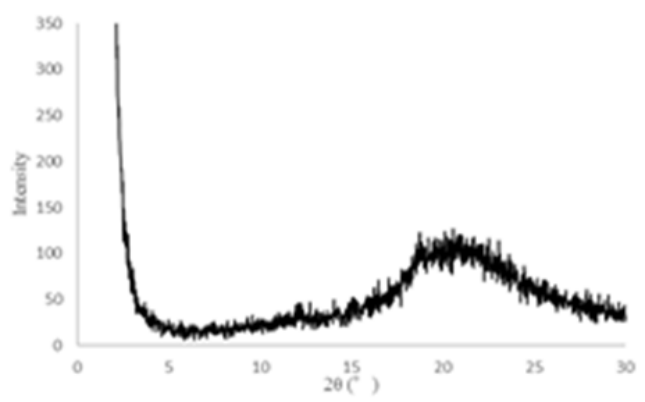

(b)

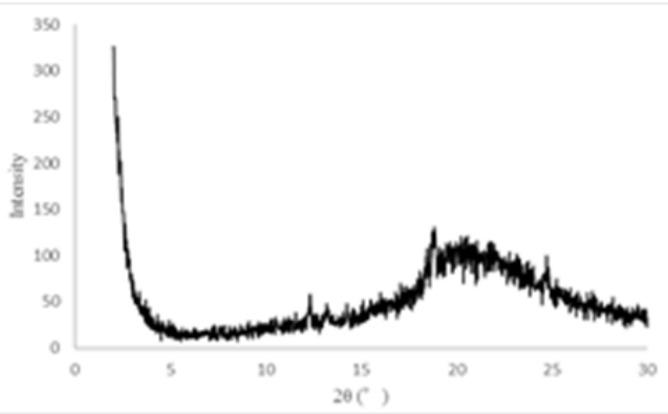

Figure S8. PXRD patterns of the 4 film measured on a glass plate. (a) Before annealing. (b) After annealing at $100^{\circ} \mathrm{C}$ for $1 \mathrm{~h}$.

(a)

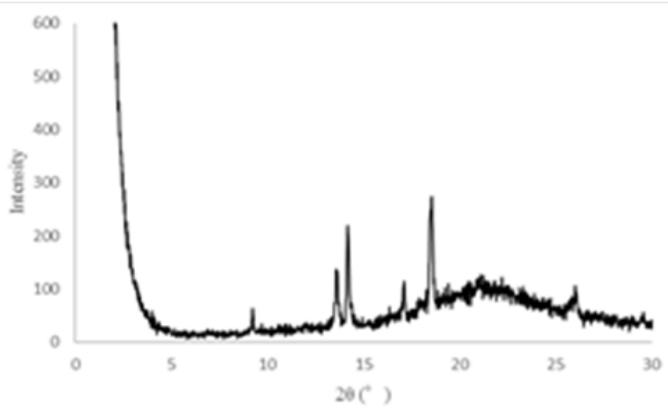

(b)

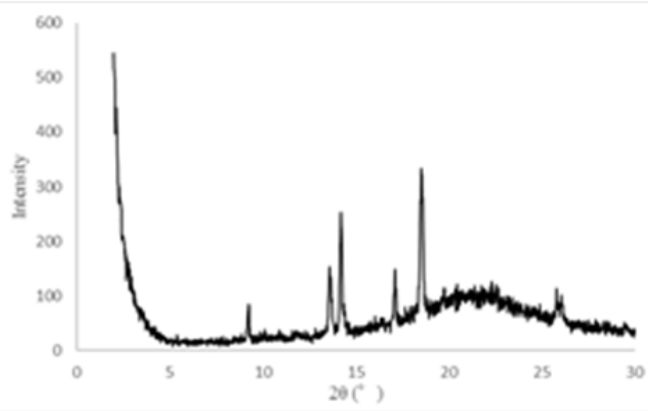




\section{S6. OFET behavior of 2,3 , and 4}

Figure S9. (Left) $I_{\mathrm{d}}-V_{\mathrm{g}}$ plots and (right) the output curve at different gate voltages for $\mathbf{2}$ before annealing.
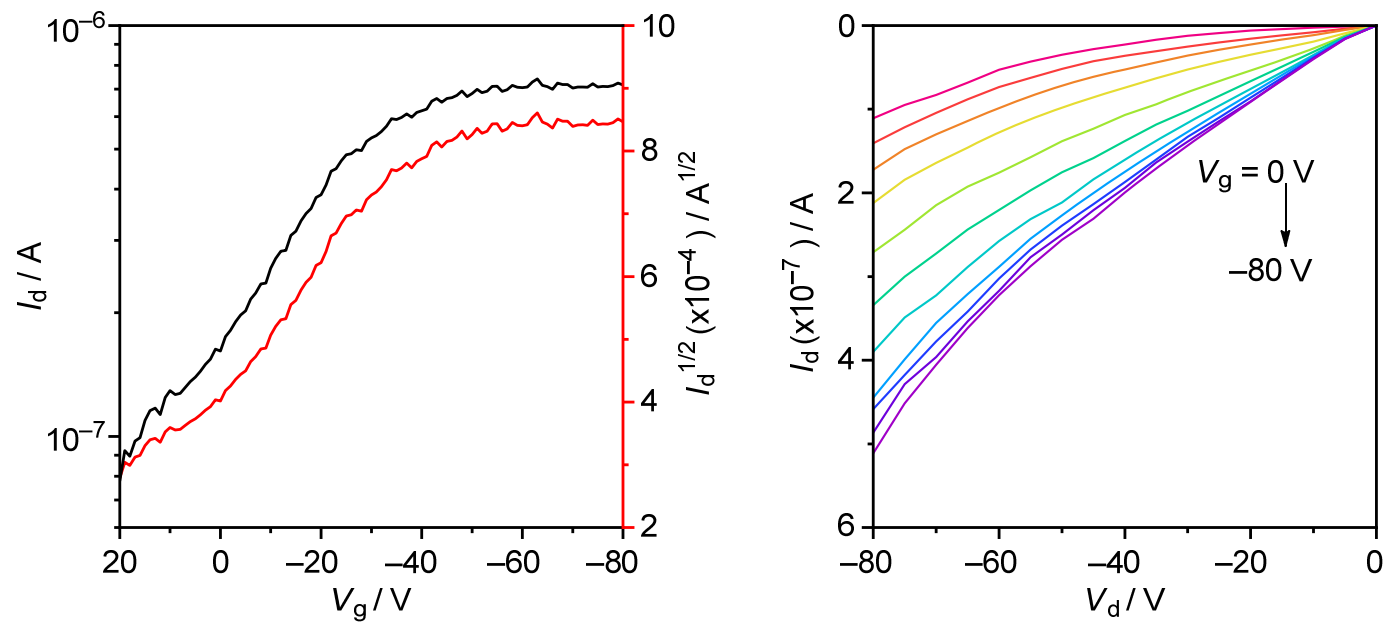

Figure S10. (Left) $I_{\mathrm{d}^{-}} V_{\mathrm{g}}$ plots and (right) the output curve at different gate voltages for $\mathbf{2}$ after annealing.
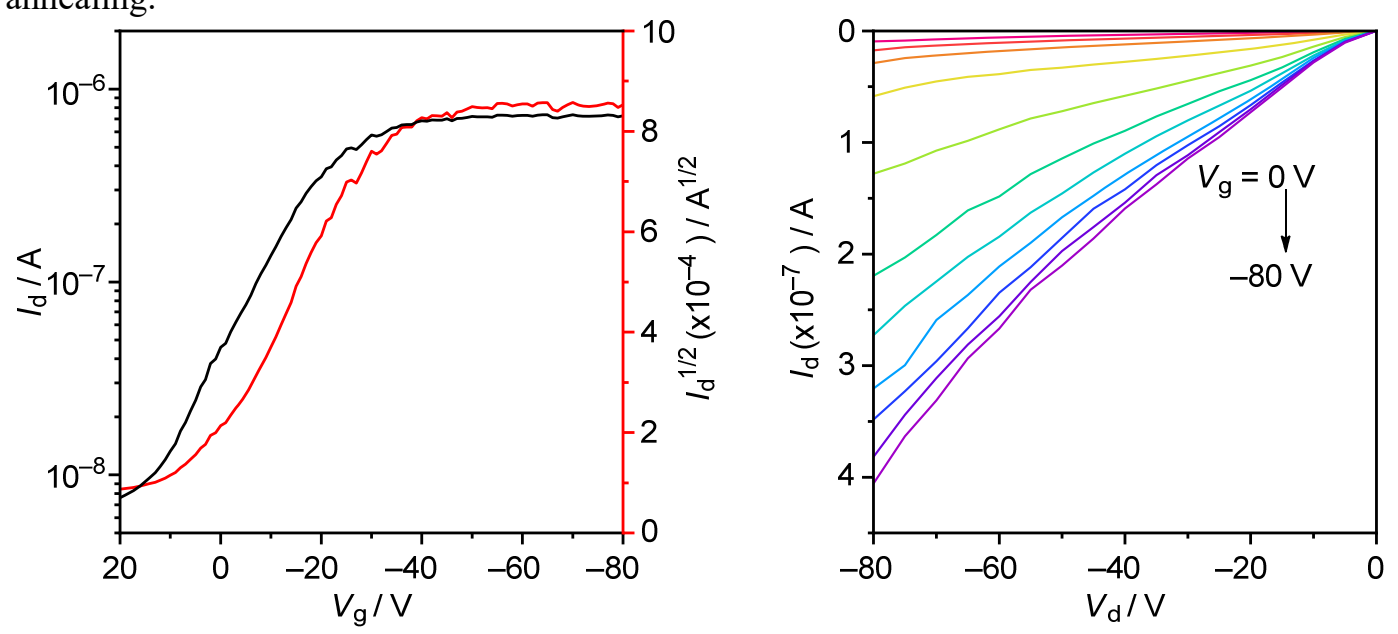

Figure S11. (Left) $I_{\mathrm{d}}-V_{\mathrm{g}}$ plots and (right) the output curve at different gate voltages for $\mathbf{3}$ before annealing.
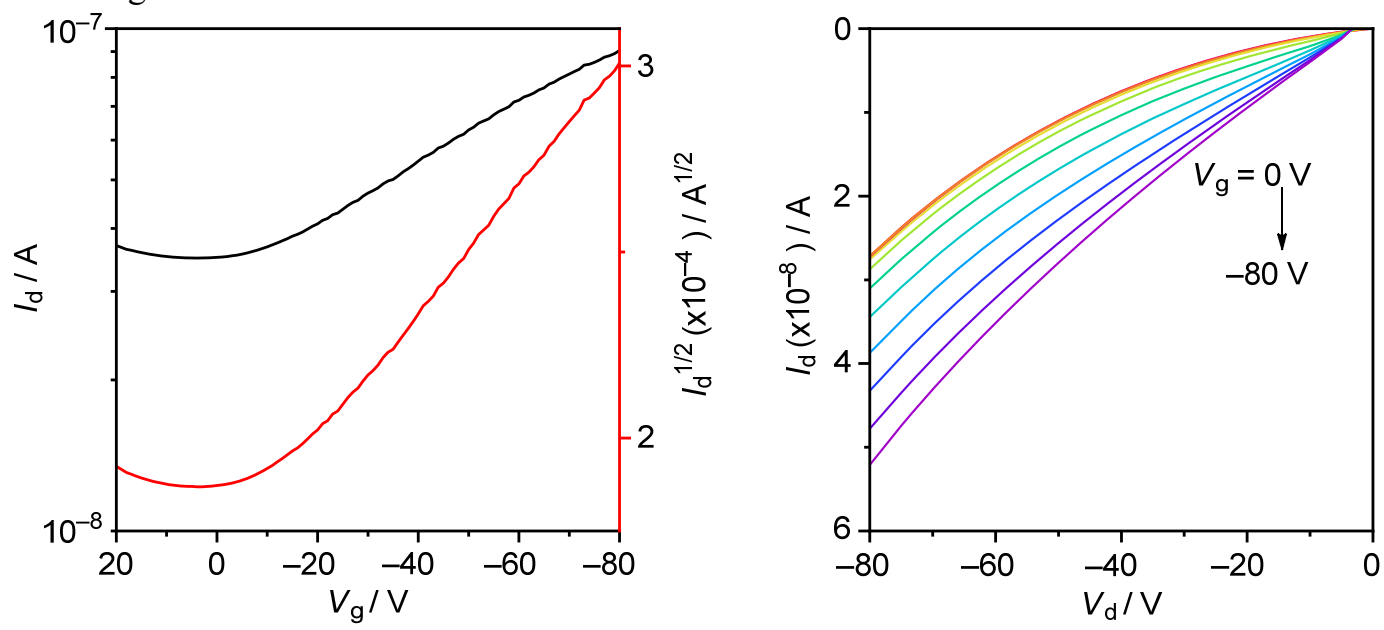
Figure S12. (Left) $I_{\mathrm{d}}-V_{\mathrm{g}}$ plots and (right) the output curve at different gate voltages for $\mathbf{3}$ after
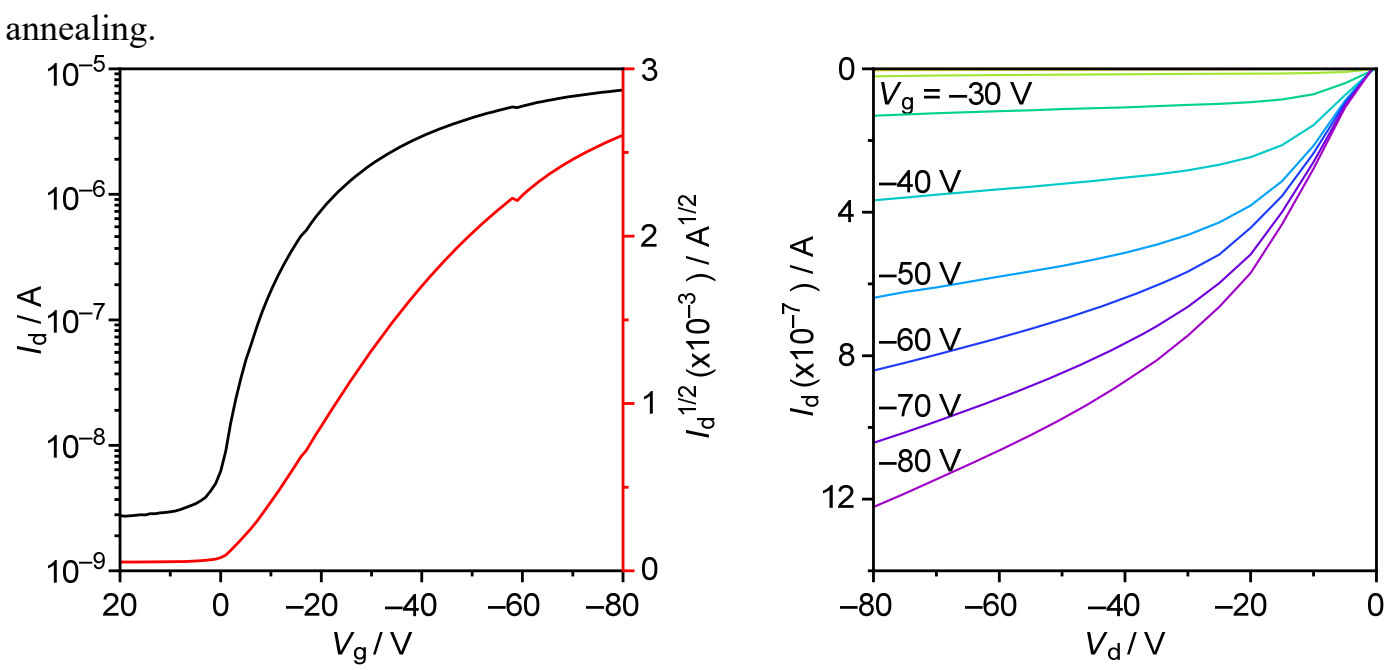

Figure S13. (Left) $I_{\mathrm{d}-} V_{\mathrm{g}}$ plots and (right) the output curve at different gate voltages for $\mathbf{4}$ before annealing.
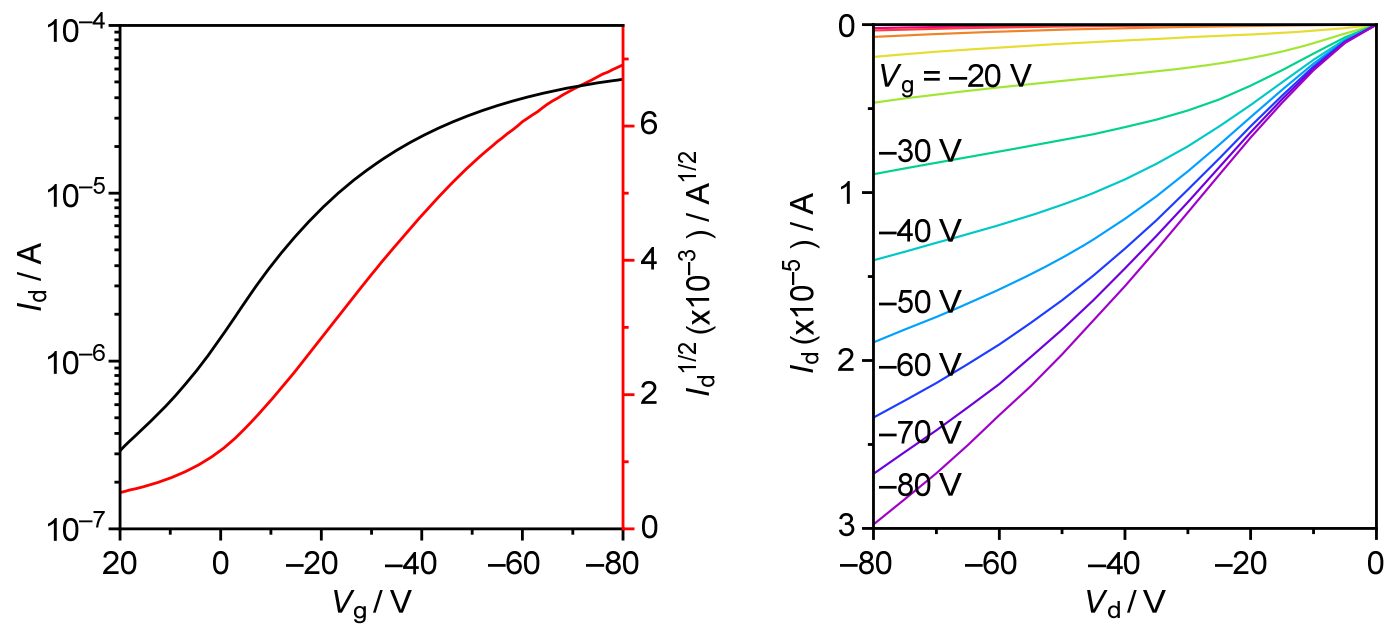

Figure S14. (Left) $I_{\mathrm{d}^{-}} V_{\mathrm{g}}$ plots and (right) the output curve at different gate voltages for 4 after annealing.
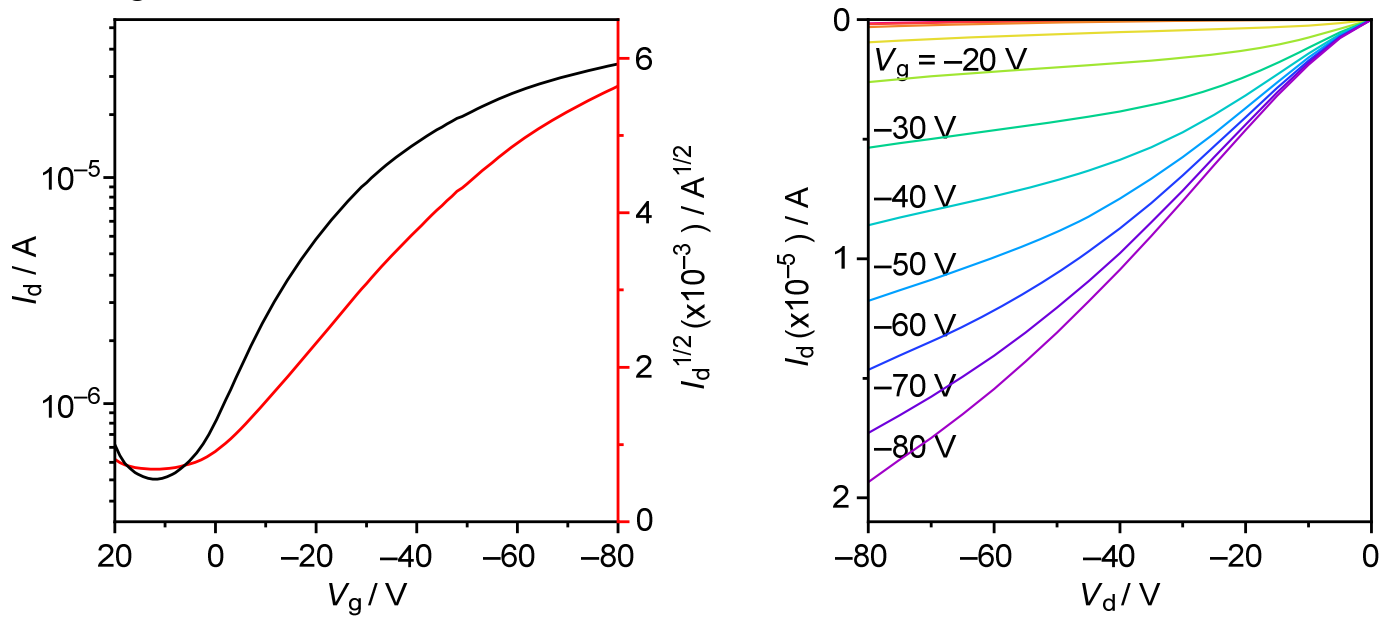


\section{S7. Possible intermediates for the McMurry reaction of 1 (theoretical studies)}

The McMurry coupling of dialdehyde $\mathbf{1}$ with low valent titanium produces $\mathrm{dl}$ - and meso-titanium pinacolates $\mathbf{1 0}$ and 11. The major $d l$-diolates $\mathbf{1 0}$ is retarded to form cyclic dimers (Figure S14). Titanium pinacolate unit is replaced by methylenedioxy group for the calculations.

Figure S15-1. Conformations of $d l$ - and meso-titanium pinacolates 10 and 11.
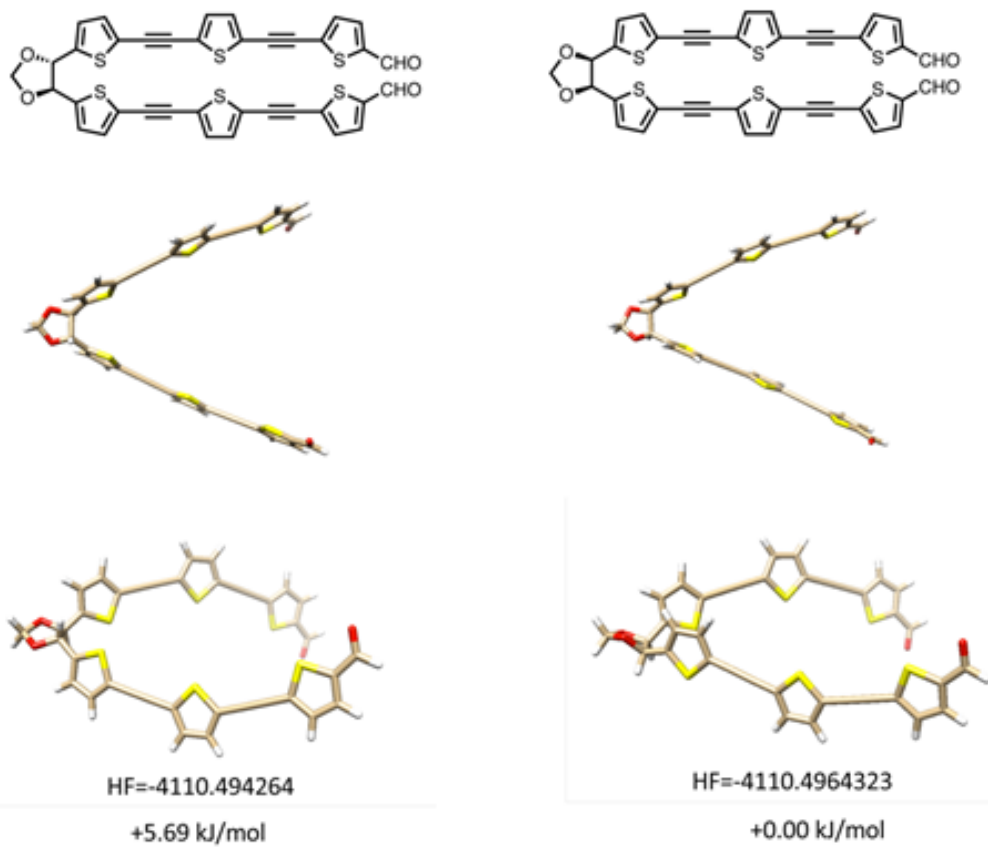

dl-titanium pinacolates 10

meso-titanium pinacolates 11

Figure S15-2. Calculated stability of cyclic intermediates anti-12 (a), syn-12 (b), and $\mathbf{1 3}$ (c).

(a)

$\mathrm{HF}=-4149.1181983$

$0.00 \mathrm{~kJ} / \mathrm{mol}$
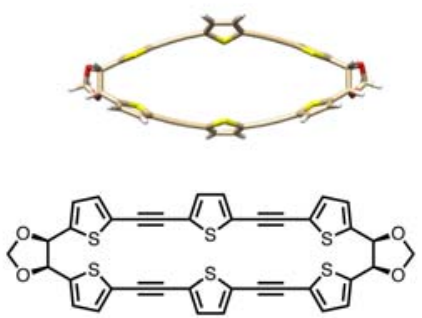

$H F=-4149.11757$

$1.65 \mathrm{~kJ} / \mathrm{mol}$
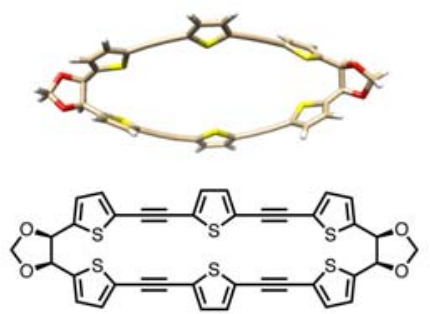

syn-12 (b)

$H F=-4149.1174699$

$+1.91 \mathrm{~kJ} / \mathrm{mol}$
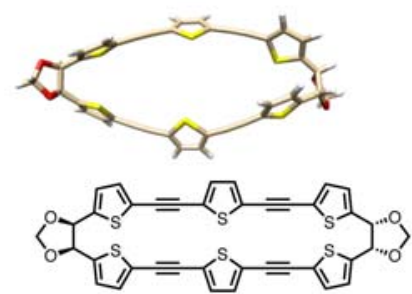

anti-12 
(c)

$H F=-4149.1146294$

$+9.37 \mathrm{~kJ} / \mathrm{mol}$
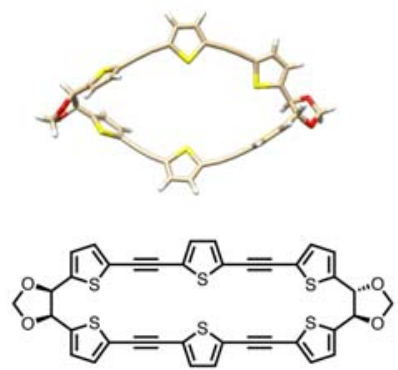

$\mathrm{HF}=-4149.1173447$

$+2.24 \mathrm{~kJ} / \mathrm{mol}$
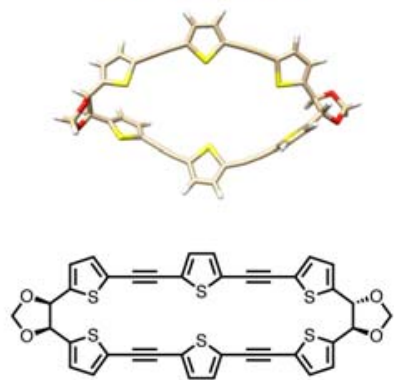

13

Table S17. Cartesian coordinate of optimized structure of $\mathbf{1 0}$ at B3LYP/6-31G(d) level.

\begin{tabular}{|c|c|c|c|c|c|}
\hline \multirow{2}{*}{$\begin{array}{l}\text { Center } \\
\text { Number }\end{array}$} & \multirow{2}{*}{$\begin{array}{l}\text { Atomic } \\
\text { Number }\end{array}$} & \multirow{2}{*}{$\begin{array}{l}\text { Atomic } \\
\text { Type }\end{array}$} & \multicolumn{3}{|c|}{ Coordinates (Angstroms) } \\
\hline & & & $\mathrm{X}$ & $\mathrm{Y}$ & Z \\
\hline 1 & 6 & 0 & -6.723703 & -3.590575 & -0.806177 \\
\hline 2 & 6 & 0 & -7.393733 & -2.161198 & -0.909043 \\
\hline 3 & 6 & 0 & -8.845740 & -3.796447 & -0.156471 \\
\hline 4 & 1 & 0 & -6.863532 & -4.077793 & -1.785638 \\
\hline 5 & 1 & 0 & -7.358541 & -1.826309 & -1.953564 \\
\hline 6 & 1 & 0 & -9.479584 & -3.887107 & 0.729681 \\
\hline 7 & 1 & 0 & -9.260831 & -4.387632 & -0.990434 \\
\hline 8 & 8 & 0 & -8.748876 & -2.430965 & -0.541651 \\
\hline 9 & 8 & 0 & -7.549197 & -4.229273 & 0.163160 \\
\hline 10 & 6 & 0 & -6.833663 & -1.100025 & -0.004829 \\
\hline 11 & 6 & 0 & -7.218237 & -0.806480 & 1.280729 \\
\hline 12 & 16 & 0 & -5.542627 & -0.054875 & -0.530068 \\
\hline 13 & 6 & 0 & -6.477943 & 0.252332 & 1.864316 \\
\hline 14 & 1 & 0 & -8.027229 & -1.327246 & 1.778234 \\
\hline 15 & 6 & 0 & -5.519250 & 0.781316 & 1.018969 \\
\hline 16 & 1 & 0 & -6.637686 & 0.629298 & 2.867949 \\
\hline 17 & 6 & 0 & -5.290757 & -3.669155 & -0.394492 \\
\hline 18 & 6 & 0 & -4.773952 & -3.671427 & 0.877464 \\
\hline 19 & 16 & 0 & -4.028637 & -3.745624 & -1.592465 \\
\hline 20 & 6 & 0 & -3.358055 & -3.728666 & 0.907594 \\
\hline 21 & 1 & 0 & -5.400669 & -3.640167 & 1.760051 \\
\hline 22 & 6 & 0 & -2.782421 & -3.765961 & -0.349746 \\
\hline 23 & 1 & 0 & -2.766679 & -3.741971 & 1.815668 \\
\hline 24 & 6 & 0 & -4.615930 & 1.825706 & 1.260330 \\
\hline 25 & 6 & 0 & -1.423655 & -3.818219 & -0.690041 \\
\hline 26 & 6 & 0 & -0.246859 & -3.861616 & -1.009823 \\
\hline 27 & 6 & 0 & -3.823773 & 2.732646 & 1.458340 \\
\hline 28 & 6 & 0 & 1.112496 & -3.910834 & -1.343838 \\
\hline 29 & 6 & 0 & 1.690059 & -3.911461 & -2.606237 \\
\hline 30 & 16 & 0 & 2.346457 & -3.981566 & -0.096008 \\
\hline 31 & 6 & 0 & 3.097169 & -3.967273 & -2.580750 \\
\hline 32 & 1 & 0 & 1.097946 & -3.871077 & -3.512867 \\
\hline 33 & 6 & 0 & 3.628167 & -4.010933 & -1.299079 \\
\hline 34 & 1 & 0 & 3.722446 & -3.975496 & -3.465875 \\
\hline 35 & 6 & 0 & -2.911420 & 3.772287 & 1.678324 \\
\hline 36 & 6 & 0 & -2.732985 & 4.535508 & 2.824392 \\
\hline 37 & 16 & 0 & -1.792203 & 4.271082 & 0.419900 \\
\hline 38 & 6 & 0 & -1.718376 & 5.504693 & 2.702455 \\
\hline
\end{tabular}




\begin{tabular}{|c|c|c|c|c|c|}
\hline 39 & 1 & 0 & -3.327486 & 4.384435 & 3.717673 \\
\hline 40 & 6 & 0 & -1.097198 & 5.505621 & 1.461189 \\
\hline 41 & 1 & 0 & -1.435012 & 6.192222 & 3.490857 \\
\hline 42 & 6 & 0 & -0.059602 & 6.329062 & 1.010094 \\
\hline 43 & 6 & 0 & 4.973461 & -4.070227 & -0.918251 \\
\hline 44 & 6 & 0 & 6.144870 & -4.121531 & -0.578706 \\
\hline 45 & 6 & 0 & 0.845734 & 7.043022 & 0.608973 \\
\hline 46 & 6 & 0 & 1.884708 & 7.858127 & 0.141556 \\
\hline 47 & 6 & 0 & 2.547699 & 8.869638 & 0.829685 \\
\hline 48 & 16 & 0 & 2.512010 & 7.679010 & -1.486536 \\
\hline 49 & 6 & 0 & 3.546974 & 9.491040 & 0.055300 \\
\hline 50 & 1 & 0 & 2.305119 & 9.134218 & 1.852024 \\
\hline 51 & 6 & 0 & 3.657993 & 8.967208 & -1.220085 \\
\hline 52 & 1 & 0 & 4.174934 & 10.301493 & 0.411716 \\
\hline 53 & 6 & 0 & 7.486961 & -4.180615 & -0.181409 \\
\hline 54 & 6 & 0 & 8.615177 & -4.190884 & -0.995689 \\
\hline 55 & 16 & 0 & 7.925545 & -4.253734 & 1.515154 \\
\hline 56 & 6 & 0 & 9.815962 & -4.256542 & -0.261997 \\
\hline 57 & 1 & 0 & 8.553557 & -4.151163 & -2.076728 \\
\hline 58 & 6 & 0 & 9.621069 & -4.296864 & 1.106818 \\
\hline 59 & 1 & 0 & 10.802952 & -4.274018 & -0.713356 \\
\hline 60 & 6 & 0 & 4.581495 & 9.375434 & -2.268420 \\
\hline 61 & 8 & 0 & 4.636629 & 8.876817 & -3.380418 \\
\hline 62 & 1 & 0 & 5.259176 & 10.205570 & -1.973160 \\
\hline 63 & 6 & 0 & 10.638725 & -4.366560 & 2.145305 \\
\hline 64 & 8 & 0 & 10.409477 & -4.400200 & 3.342956 \\
\hline 65 & 1 & 0 & 11.679349 & -4.387868 & 1.755262 \\
\hline
\end{tabular}

Compound: 10 (See Figure S14)

Method: B3LYP/6-31G(d)

Key word: opt freq scf=(direct, tight)

Symmetry: $C_{1}$

\# of imaginary frequencies: 0

Energy:-4110.494264 Hartree

Table S18. Cartesian coordinate of optimized structure of 11 at B3LYP/6-31G(d) level.

\begin{tabular}{rrrrrr}
$\begin{array}{c}\text { Center } \\
\text { Number }\end{array}$ & $\begin{array}{c}\text { Atomic } \\
\text { Number }\end{array}$ & $\begin{array}{c}\text { Atomic } \\
\text { Type }\end{array}$ & \multicolumn{2}{c}{ Coordinates (Angstroms) } \\
\hline 1 & 6 & 0 & -7.367787 & 0.772271 & Y \\
2 & 6 & 0 & -7.367369 & -0.775226 & 0.099412 \\
3 & 6 & 0 & -9.524290 & -0.002068 & 0.000152 \\
4 & 1 & 0 & -7.228470 & 0.977820 & -1.171844 \\
5 & 1 & 0 & -7.227876 & -0.980718 & 1.171842 \\
6 & 1 & 0 & -10.153248 & 0.203031 & -0.876743 \\
7 & 1 & 0 & -10.152915 & -0.207519 & 0.877207 \\
8 & 8 & 0 & -8.692067 & -1.106998 & -0.312745 \\
9 & 8 & 0 & -8.692619 & 1.103358 & 0.312843 \\
10 & 6 & 0 & -6.381764 & -1.541907 & -0.723988 \\
11 & 6 & 0 & -6.494474 & -1.907718 & -2.044271 \\
12 & 16 & 0 & -4.862852 & -2.061452 & -0.050876 \\
13 & 6 & 0 & -5.361154 & -2.603181 & -2.533440 \\
14 & 1 & 0 & -7.380140 & -1.698739 & -2.632617 \\
15 & 6 & 0 & -4.370396 & -2.773827 & -1.582148 \\
16 & 1 & 0 & -5.262971 & -2.976044 & -3.546345 \\
17 & 6 & 0 & -6.382525 & 1.539483 & 0.723928 \\
18 & 6 & 0 & -6.495429 & 1.905400 & 2.044167 \\
19 & 16 & 0 & -4.863829 & 2.059616 & 0.050799 \\
20 & 6 & 0 & -5.362424 & 2.601408 & 2.533283 \\
21 & 1 & 0 & -7.381017 & 1.696094 & 2.632517 \\
22 & 6 & 0 & -4.371718 & 2.772379 & 1.581991 \\
& & & & &
\end{tabular}




\begin{tabular}{|c|c|c|c|c|c|}
\hline 23 & 1 & 0 & -5.264420 & 2.974426 & 3.546149 \\
\hline 24 & 6 & 0 & -3.124911 & -3.403301 & -1.716357 \\
\hline 25 & 6 & 0 & -3.126498 & 3.402389 & 1.716168 \\
\hline 26 & 6 & 0 & -2.039690 & 3.946888 & 1.822500 \\
\hline 27 & 6 & 0 & -2.037867 & -3.947330 & -1.822679 \\
\hline 28 & 6 & 0 & -0.788543 & -4.570166 & -1.937457 \\
\hline 29 & 6 & 0 & -0.213009 & -5.147096 & -3.061511 \\
\hline 30 & 16 & 0 & 0.284137 & -4.702515 & -0.553079 \\
\hline 31 & 6 & 0 & 1.065742 & -5.687406 & -2.823930 \\
\hline 32 & 1 & 0 & -0.715254 & -5.168831 & -4.021444 \\
\hline 33 & 6 & 0 & 1.497835 & -5.536210 & -1.513559 \\
\hline 34 & 1 & 0 & 1.669982 & -6.176884 & -3.578795 \\
\hline 35 & 6 & 0 & -0.790623 & 4.570237 & 1.937298 \\
\hline 36 & 6 & 0 & -0.215423 & 5.147596 & 3.061303 \\
\hline 37 & 16 & 0 & 0.282159 & 4.702732 & 0.553007 \\
\hline 38 & 6 & 0 & 1.063144 & 5.688356 & 2.823753 \\
\hline 39 & 1 & 0 & -0.717771 & 5.169311 & 4.021183 \\
\hline 40 & 6 & 0 & 1.495428 & 5.537087 & 1.513453 \\
\hline 41 & 1 & 0 & 1.667122 & 6.178198 & 3.578592 \\
\hline 42 & 6 & 0 & 2.707039 & -5.951952 & -0.944911 \\
\hline 43 & 6 & 0 & 2.704530 & 5.953186 & 0.944851 \\
\hline 44 & 6 & 0 & 3.757045 & 6.312793 & 0.441829 \\
\hline 45 & 6 & 0 & 3.759645 & -6.311225 & -0.441842 \\
\hline 46 & 6 & 0 & 4.965390 & -6.720342 & 0.142388 \\
\hline 47 & 6 & 0 & 6.029044 & -7.374320 & -0.471654 \\
\hline 48 & 16 & 0 & 5.291327 & -6.430734 & 1.841183 \\
\hline 49 & 6 & 0 & 7.091520 & -7.637894 & 0.414984 \\
\hline 50 & 1 & 0 & 6.020060 & -7.642296 & -1.521480 \\
\hline 51 & 6 & 0 & 6.854382 & -7.193118 & 1.703095 \\
\hline 52 & 1 & 0 & 8.010789 & -8.139174 & 0.128888 \\
\hline 53 & 6 & 0 & 4.962695 & 6.722276 & -0.142340 \\
\hline 54 & 6 & 0 & 6.025934 & 7.376979 & 0.471647 \\
\hline 55 & 16 & 0 & 5.289051 & 6.432225 & -1.840978 \\
\hline 56 & 6 & 0 & 7.088429 & 7.640749 & -0.414908 \\
\hline 57 & 1 & 0 & 6.016648 & 7.645322 & 1.521377 \\
\hline 58 & 6 & 0 & 6.851714 & 7.195414 & -1.702904 \\
\hline 59 & 1 & 0 & 8.007411 & 8.142571 & -0.128837 \\
\hline 60 & 6 & 0 & 7.736871 & -7.303880 & 2.855401 \\
\hline 61 & 8 & 0 & 7.476001 & -6.892532 & 3.973793 \\
\hline 62 & 1 & 0 & 8.702338 & -7.809170 & 2.636127 \\
\hline 63 & 6 & 0 & 7.734334 & 7.306198 & -2.855108 \\
\hline 64 & 8 & 0 & 7.473856 & 6.894303 & -3.973390 \\
\hline 65 & 1 & 0 & 8.699505 & 7.812068 & -2.635866 \\
\hline
\end{tabular}

Compound: 11 (See Figure S14)

Method: B3LYP/6-31G(d)

Key word: opt freq $\mathrm{scf}=$ (direct, tight)

Symmetry: $C_{1}$

\# of imaginary frequencies: 0

Energy: -4110.4964323 Hartree 
Table S19. Cartesian coordinate of optimized structure of syn-12 at B3LYP/6-31G(d) level.

\begin{tabular}{|c|c|c|c|c|c|}
\hline \multirow{2}{*}{$\begin{array}{l}\text { Center } \\
\text { Number }\end{array}$} & \multirow{2}{*}{$\begin{array}{l}\text { Atomic } \\
\text { Number }\end{array}$} & \multirow{2}{*}{$\begin{array}{l}\text { Atomic } \\
\text { Type }\end{array}$} & \multicolumn{3}{|c|}{ Coordinates (Angstroms) } \\
\hline & & & $\mathrm{X}$ & $\mathrm{Y}$ & $\mathrm{Z}$ \\
\hline 1 & 6 & 0 & 1.290521 & -3.287573 & -0.305976 \\
\hline 2 & 6 & 0 & 0.709292 & -3.710234 & -1.486945 \\
\hline 3 & 6 & 0 & -0.709289 & -3.710085 & -1.486898 \\
\hline 4 & 6 & 0 & -1.290352 & -3.287290 & -0.305895 \\
\hline 5 & 16 & 0 & 0.000170 & -2.866984 & 0.917525 \\
\hline 6 & 6 & 0 & 2.639202 & -3.089824 & 0.003082 \\
\hline 7 & 6 & 0 & 3.800044 & -2.834740 & 0.299784 \\
\hline 8 & 6 & 0 & 5.111170 & -2.458603 & 0.610615 \\
\hline 9 & 6 & 0 & -5.111007 & -2.458292 & 0.610800 \\
\hline 10 & 16 & 0 & 6.189746 & -1.735638 & -0.676780 \\
\hline 11 & 6 & 0 & 7.477204 & -1.485271 & 0.560883 \\
\hline 12 & 6 & 0 & 7.084815 & -1.949345 & 1.790729 \\
\hline 13 & 6 & 0 & 5.764890 & -2.486730 & 1.825045 \\
\hline 14 & 6 & 0 & -5.764814 & -2.486519 & 1.825180 \\
\hline 15 & 6 & 0 & -7.084809 & -1.949311 & 1.790770 \\
\hline 16 & 6 & 0 & -7.477170 & -1.485283 & 0.560898 \\
\hline 17 & 16 & 0 & -6.189580 & -1.735451 & -0.676669 \\
\hline 18 & 6 & 0 & 1.290971 & 3.354889 & 0.579874 \\
\hline 19 & 6 & 0 & 0.709338 & 3.901437 & 1.708639 \\
\hline 20 & 6 & 0 & -0.709430 & 3.901440 & 1.708667 \\
\hline 21 & 6 & 0 & -1.291109 & 3.354904 & 0.579919 \\
\hline 22 & 16 & 0 & -0.000093 & 2.801438 & -0.589265 \\
\hline 23 & 6 & 0 & -2.640108 & 3.133318 & 0.288175 \\
\hline 24 & 6 & 0 & -3.800293 & 2.858742 & 0.005602 \\
\hline 25 & 6 & 0 & 5.110786 & 2.469950 & -0.288595 \\
\hline 26 & 6 & 0 & -5.110914 & 2.469851 & -0.288485 \\
\hline 27 & 16 & 0 & 6.186525 & 1.779911 & 1.025574 \\
\hline 28 & 6 & 0 & 7.472883 & 1.458416 & -0.207491 \\
\hline 29 & 6 & 0 & 7.082118 & 1.881509 & -1.448886 \\
\hline 30 & 6 & 0 & 5.769838 & 2.439612 & -1.498658 \\
\hline 31 & 6 & 0 & -5.769907 & 2.439552 & -1.498580 \\
\hline 32 & 6 & 0 & -7.082185 & 1.881434 & -1.448894 \\
\hline 33 & 6 & 0 & -7.473009 & 1.458305 & -0.207530 \\
\hline 34 & 16 & 0 & -6.186709 & 1.779754 & 1.025607 \\
\hline 35 & 6 & 0 & -8.742220 & -0.789276 & 0.224090 \\
\hline 36 & 6 & 0 & -8.750763 & 0.795098 & 0.192874 \\
\hline 37 & 6 & 0 & 8.750633 & 0.795253 & 0.193002 \\
\hline 38 & 6 & 0 & 8.742201 & -0.789124 & 0.224161 \\
\hline 39 & 6 & 0 & -2.638970 & -3.089236 & 0.003246 \\
\hline 40 & 6 & 0 & -3.799795 & -2.834217 & 0.300073 \\
\hline 41 & 6 & 0 & 3.800158 & 2.858868 & 0.005419 \\
\hline 42 & 6 & 0 & 2.639958 & 3.133291 & 0.288082 \\
\hline 43 & 8 & 0 & 9.791104 & 1.092792 & -0.804582 \\
\hline 44 & 8 & 0 & 9.154990 & -1.120759 & -1.145020 \\
\hline 45 & 8 & 0 & -9.791161 & 1.092537 & -0.804823 \\
\hline 46 & 8 & 0 & -9.154915 & -1.120994 & -1.145097 \\
\hline 47 & 6 & 0 & -10.157123 & -0.147939 & -1.491905 \\
\hline 48 & 6 & 0 & 10.157134 & -0.147616 & -1.491760 \\
\hline 49 & 1 & 0 & 1.305022 & -4.003597 & -2.342041 \\
\hline 50 & 1 & 0 & -1.305136 & -4.003324 & -2.341955 \\
\hline 51 & 1 & 0 & 7.723979 & -1.900157 & 2.665069 \\
\hline 52 & 1 & 0 & 5.300689 & -2.880602 & 2.720071 \\
\hline 53 & 1 & 0 & -5.300625 & -2.880326 & 2.720241 \\
\hline 54 & 1 & 0 & -7.724041 & -1.900207 & 2.665065 \\
\hline 55 & 1 & 0 & 1.305015 & 4.287989 & 2.525936 \\
\hline 56 & 1 & 0 & -1.305074 & 4.287993 & 2.525987 \\
\hline 57 & 1 & 0 & 7.729399 & 1.800850 & -2.311564 \\
\hline 58 & 1 & 0 & 5.309895 & 2.804767 & -2.408278 \\
\hline 59 & 1 & 0 & -5.309917 & 2.804732 & -2.408166 \\
\hline
\end{tabular}




$\begin{array}{lllrrr}60 & 1 & 0 & -7.729422 & 1.800788 & -2.311606 \\ 61 & 1 & 0 & -9.528818 & -1.103717 & 0.926800 \\ 62 & 1 & 0 & -9.096823 & 1.165848 & 1.162907 \\ 63 & 1 & 0 & 9.096595 & 1.165998 & 1.163071 \\ 64 & 1 & 0 & 9.528794 & -1.103530 & 0.926892 \\ 65 & 1 & 0 & -10.131844 & 0.002664 & -2.570378 \\ 66 & 1 & 0 & -11.150481 & -0.461441 & -1.148146 \\ 67 & 1 & 0 & 10.131841 & 0.003061 & -2.570223 \\ 68 & 1 & 0 & 11.150514 & -0.461072 & -1.148027\end{array}$

Compound: syn-12 (See Figure S15a) Method: B3LYP/6-31G(d)

Key word: opt freq scf=(direct, tight)

Symmetry: $C_{1}$

\# of imaginary frequencies: 0
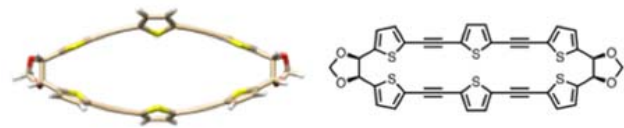

Energy: -4149.1181983 Hartree

Table S20. Cartesian coordinate of optimized structure of syn-12 at B3LYP/6-31G(d) level.

\begin{tabular}{|c|c|c|c|c|c|}
\hline \multirow{2}{*}{$\begin{array}{l}\text { Center } \\
\text { Number }\end{array}$} & \multirow{2}{*}{$\begin{array}{l}\text { Atomic } \\
\text { Number }\end{array}$} & \multirow{2}{*}{$\begin{array}{l}\text { Atomic } \\
\text { Type }\end{array}$} & \multicolumn{3}{|c|}{ Coordinates (Angstroms) } \\
\hline & & & $\mathrm{X}$ & $\mathrm{Y}$ & Z \\
\hline 1 & 6 & 0 & 1.204009 & 3.539210 & 0.177147 \\
\hline 2 & 6 & 0 & 0.740476 & 4.442745 & 1.114487 \\
\hline 3 & 6 & 0 & -0.670271 & 4.484553 & 1.259534 \\
\hline 4 & 6 & 0 & -1.363310 & 3.613435 & 0.438440 \\
\hline 5 & 16 & 0 & -0.201014 & 2.667694 & -0.604656 \\
\hline 6 & 6 & 0 & 2.516380 & 3.213225 & -0.180319 \\
\hline 7 & 6 & 0 & 3.644197 & 2.868484 & -0.511676 \\
\hline 8 & 6 & 0 & 4.928820 & 2.434288 & -0.860800 \\
\hline 9 & 6 & 0 & -5.257057 & 2.665335 & 0.188306 \\
\hline 10 & 16 & 0 & 6.097519 & 1.865586 & 0.427013 \\
\hline 11 & 6 & 0 & 7.311304 & 1.497944 & -0.854262 \\
\hline 12 & 6 & 0 & 6.832964 & 1.812003 & -2.100824 \\
\hline 13 & 6 & 0 & 5.503546 & 2.327056 & -2.109527 \\
\hline 14 & 6 & 0 & -6.355145 & 3.027042 & 0.940662 \\
\hline 15 & 6 & 0 & -7.556968 & 2.332998 & 0.607405 \\
\hline 16 & 6 & 0 & -7.424425 & 1.414689 & -0.399575 \\
\hline 17 & 16 & 0 & -5.729541 & 1.390739 & -1.036847 \\
\hline 18 & 6 & 0 & 1.419888 & -3.718876 & -0.147246 \\
\hline 19 & 6 & 0 & 0.847421 & -4.613690 & -1.031055 \\
\hline 20 & 6 & 0 & -0.572103 & -4.631449 & -1.028995 \\
\hline 21 & 6 & 0 & -1.161260 & -3.749623 & -0.142393 \\
\hline 22 & 16 & 0 & 0.118910 & -2.826379 & 0.776329 \\
\hline 23 & 6 & 0 & -2.509145 & -3.447288 & 0.070869 \\
\hline 24 & 6 & 0 & -3.655390 & -3.066234 & 0.275529 \\
\hline 25 & 6 & 0 & 5.199090 & -2.506429 & 0.466612 \\
\hline 26 & 6 & 0 & -4.933751 & -2.541062 & 0.483767 \\
\hline 27 & 16 & 0 & 6.171740 & -1.877157 & -0.954716 \\
\hline 28 & 6 & 0 & 7.484341 & -1.364448 & 0.180994 \\
\hline 29 & 6 & 0 & 7.172617 & -1.687772 & 1.473624 \\
\hline 30 & 6 & 0 & 5.902283 & -2.317356 & 1.636101 \\
\hline 31 & 6 & 0 & -5.461034 & -1.964074 & 1.619663 \\
\hline 32 & 6 & 0 & -6.757748 & -1.394644 & 1.452705 \\
\hline 33 & 6 & 0 & -7.270672 & -1.523300 & 0.191460 \\
\hline 34 & 16 & 0 & -6.136861 & -2.423773 & -0.894119 \\
\hline 35 & 6 & 0 & -8.459172 & 0.502761 & -0.977410 \\
\hline 36 & 6 & 0 & -8.528806 & -0.954363 & -0.354365 \\
\hline 37 & 6 & 0 & 8.707647 & -0.684135 & -0.341824 \\
\hline 38 & 6 & 0 & 8.621477 & 0.886957 & -0.521575 \\
\hline 39 & 6 & 0 & -2.734707 & 3.361544 & 0.352275 \\
\hline 40 & 6 & 0 & -3.923107 & 3.072496 & 0.277925 \\
\hline 41 & 6 & 0 & 3.908471 & -3.009111 & 0.270126 \\
\hline
\end{tabular}




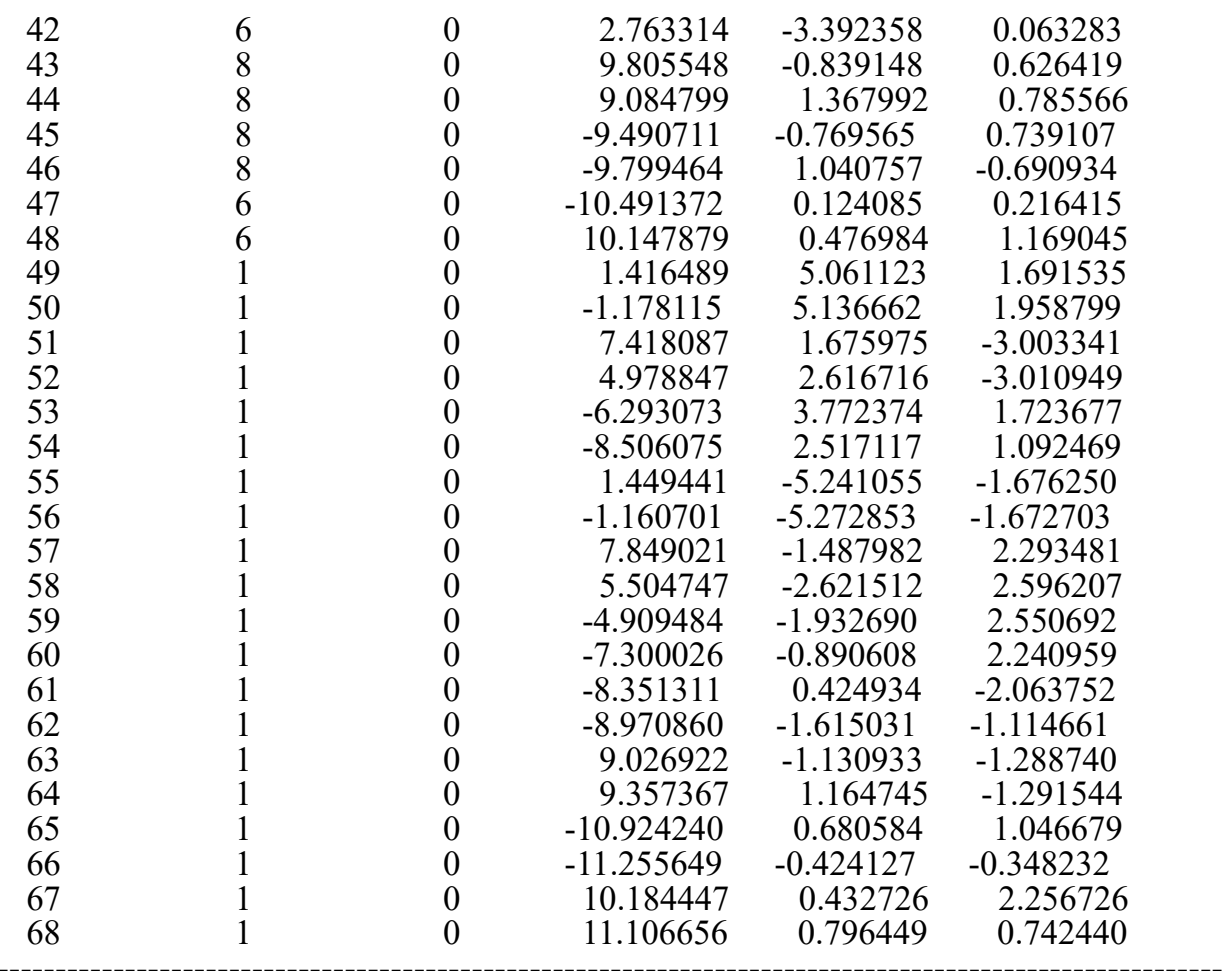

Compound: syn-12 (See Figure S15a) Method: B3LYP/6-31G(d)

Key word: opt freq scf=(direct, tight) Symmetry: $C_{1}$

\# of imaginary frequencies: 0

Energy: -4149.11757 Hartree
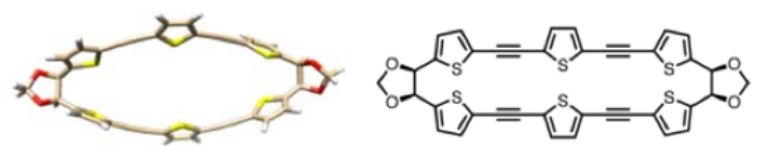

Table S21. Cartesian coordinate of optimized structure of anti-12 at B3LYP/6-31G(d) level.

\begin{tabular}{cccccc}
$\begin{array}{c}\text { Center } \\
\text { Number }\end{array}$ & $\begin{array}{c}\text { Atomic } \\
\text { Number }\end{array}$ & $\begin{array}{c}\text { Atomic } \\
\text { Type }\end{array}$ & $\mathrm{X}$ & $\mathrm{Y}$ & $\mathrm{Z}$ \\
\hline 1 & 6 & 0 & -1.322000 & -3.627827 & -0.588953 \\
2 & 6 & 0 & -0.573094 & -4.590328 & -1.240906 \\
3 & 6 & 0 & 0.818244 & -4.551317 & -0.965974 \\
4 & 6 & 0 & 1.209181 & -3.556491 & -0.088815 \\
5 & 16 & 0 & -0.242671 & -2.591226 & 0.458913 \\
6 & 6 & 0 & -2.686142 & -3.338739 & -0.677866 \\
7 & 6 & 0 & -3.860398 & -3.001477 & -0.768980 \\
8 & 6 & 0 & -5.181663 & -2.558390 & -0.885219 \\
9 & 6 & 0 & 4.792865 & -2.198376 & 1.102152 \\
10 & 16 & 0 & -5.986101 & -1.682584 & 0.502815 \\
11 & 6 & 0 & -7.457979 & -1.446321 & -0.514419 \\
12 & 6 & 0 & -7.305462 & -2.023982 & -1.749953 \\
13 & 6 & 0 & -6.037869 & -2.637492 & -1.964730 \\
14 & 6 & 0 & 5.085181 & -1.468004 & 2.235995 \\
15 & 6 & 0 & 6.413557 & -0.953272 & 2.275018 \\
16 & 6 & 0 & 7.173735 & -1.262044 & 1.176740 \\
17 & 16 & 0 & 6.258653 & -2.292435 & 0.012631 \\
18 & 6 & 0 & -1.133829 & 3.521999 & -0.716073 \\
19 & 6 & 0 & -0.604548 & 4.197368 & -1.799503 \\
20 & 6 & 0 & 0.814040 & 4.255234 & -1.830353 \\
21 & 6 & 0 & 1.445511 & 3.630443 & -0.773239
\end{tabular}




\begin{tabular}{|c|c|c|c|c|c|}
\hline 22 & 16 & 0 & 0.206222 & 2.913012 & 0.364303 \\
\hline 23 & 6 & 0 & 2.809746 & 3.443615 & -0.519444 \\
\hline 24 & 6 & 0 & 3.979118 & 3.199742 & -0.248442 \\
\hline 25 & 6 & 0 & -4.882474 & 2.484914 & 0.290866 \\
\hline 26 & 6 & 0 & 5.296174 & 2.815043 & 0.037303 \\
\hline 27 & 16 & 0 & -6.080604 & 1.902787 & -0.967139 \\
\hline 28 & 6 & 0 & -7.255683 & 1.500053 & 0.350471 \\
\hline 29 & 6 & 0 & -6.747389 & 1.823773 & 1.579440 \\
\hline 30 & 6 & 0 & -5.428801 & 2.365663 & 1.550892 \\
\hline 31 & 6 & 0 & 6.191054 & 3.326586 & 0.951855 \\
\hline 32 & 6 & 0 & 7.433282 & 2.626648 & 1.010992 \\
\hline 33 & 6 & 0 & 7.522599 & 1.556599 & 0.156669 \\
\hline 34 & 16 & 0 & 6.025406 & 1.377655 & -0.828512 \\
\hline 35 & 6 & 0 & 8.544549 & -0.776342 & 0.851336 \\
\hline 36 & 6 & 0 & 8.641013 & 0.587779 & 0.039841 \\
\hline 37 & 6 & 0 & -8.579613 & 0.889017 & 0.024497 \\
\hline 38 & 6 & 0 & -8.634472 & -0.689533 & -0.026054 \\
\hline 39 & 6 & 0 & 2.485285 & -3.186066 & 0.343144 \\
\hline 40 & 6 & 0 & 3.571647 & -2.759543 & 0.716035 \\
\hline 41 & 6 & 0 & -3.601061 & 2.906526 & -0.077751 \\
\hline 42 & 6 & 0 & -2.464784 & 3.232295 & -0.399271 \\
\hline 43 & 8 & 0 & -9.543772 & 1.202872 & 1.092705 \\
\hline 44 & 8 & 0 & -8.912231 & -1.021299 & 1.379523 \\
\hline 45 & 8 & 0 & 8.768312 & 0.121120 & -1.344384 \\
\hline 46 & 8 & 0 & 9.228380 & -1.736902 & -0.029203 \\
\hline 47 & 6 & 0 & -9.853697 & -0.028347 & 1.825994 \\
\hline 48 & 6 & 0 & 9.587585 & -1.061060 & -1.274671 \\
\hline 49 & 1 & 0 & -1.026832 & -5.307139 & -1.913676 \\
\hline 50 & 1 & 0 & 1.532280 & -5.234419 & -1.408331 \\
\hline 51 & 1 & 0 & -8.085998 & -2.004308 & -2.502147 \\
\hline 52 & 1 & 0 & -5.752970 & -3.117645 & -2.892040 \\
\hline 53 & 1 & 0 & 4.352504 & -1.298566 & 3.014806 \\
\hline 54 & 1 & 0 & 6.785604 & -0.346035 & 3.092077 \\
\hline 55 & 1 & 0 & -1.235478 & 4.636442 & -2.561907 \\
\hline 56 & 1 & 0 & 1.372588 & 4.740761 & -2.620722 \\
\hline 57 & 1 & 0 & -7.313948 & 1.685274 & 2.490247 \\
\hline 58 & 1 & 0 & -4.883700 & 2.659615 & 2.438968 \\
\hline 59 & 1 & 0 & 5.956736 & 4.184615 & 1.569149 \\
\hline 60 & 1 & 0 & 8.239112 & 2.911546 & 1.678208 \\
\hline 61 & 1 & 0 & 9.123593 & -0.671842 & 1.775560 \\
\hline 62 & 1 & 0 & 9.578420 & 1.078151 & 0.344008 \\
\hline 63 & 1 & 0 & -8.976898 & 1.290507 & -0.912459 \\
\hline 64 & 1 & 0 & -9.508105 & -0.959388 & -0.638972 \\
\hline 65 & 1 & 0 & -9.709862 & 0.125609 & 2.895001 \\
\hline 66 & 1 & 0 & -10.884317 & -0.322497 & 1.593170 \\
\hline 67 & 1 & 0 & 9.343620 & -1.702946 & -2.118660 \\
\hline 68 & 1 & 0 & 10.653211 & -0.799483 & -1.251306 \\
\hline
\end{tabular}

Compound: anti-12 (See Figure S15b)

Method: B3LYP/6-31G(d)

Key word: opt freq scf=(direct, tight)

Symmetry: $C_{1}$

\# of imaginary frequencies: 0
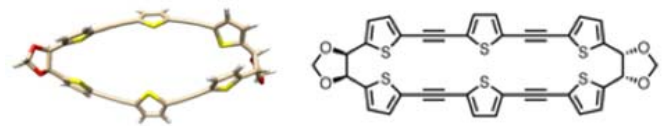

Energy: -4149.1174699 Hartree

Table S22. Cartesian coordinate of optimized structure of 13-i at B3LYP/6-31G(d) level.

\begin{tabular}{cccccc}
$\begin{array}{c}\text { Center } \\
\text { Number }\end{array}$ & $\begin{array}{c}\text { Atomic } \\
\text { Number }\end{array}$ & $\begin{array}{c}\text { Atomic } \\
\text { Type }\end{array}$ & X & Coordinates (Angstroms) & $\mathrm{Z}$ \\
\hline 1 & 6 & 0 & -1.433895 & -4.136951 & 0.028083 \\
2 & 6 & 0 & -1.087191 & -5.229358 & 0.799017 \\
3 & 6 & 0 & 0.293957 & -5.316010 & 1.124702
\end{tabular}




\begin{tabular}{|c|c|c|c|c|c|}
\hline 4 & 6 & 0 & 1.074524 & -4.295072 & 0.619180 \\
\hline 5 & 16 & 0 & 0.046672 & -3.134731 & -0.348842 \\
\hline 6 & 6 & 0 & -2.680060 & -3.681659 & -0.417358 \\
\hline 7 & 6 & 0 & -3.697377 & -3.127619 & -0.816489 \\
\hline 8 & 6 & 0 & -4.812912 & -2.400154 & -1.247585 \\
\hline 9 & 6 & 0 & 4.805305 & -2.931515 & 1.119144 \\
\hline 10 & 16 & 0 & -6.145747 & -1.939430 & -0.080683 \\
\hline 11 & 6 & 0 & -6.916309 & -0.930440 & -1.372182 \\
\hline 12 & 6 & 0 & -6.202367 & -1.006669 & -2.540868 \\
\hline 13 & 6 & 0 & -5.032272 & -1.816033 & -2.477065 \\
\hline 14 & 6 & 0 & 5.395080 & -2.478702 & 2.280035 \\
\hline 15 & 6 & 0 & 6.579853 & -1.713354 & 2.078364 \\
\hline 16 & 6 & 0 & 6.947433 & -1.556773 & 0.766984 \\
\hline 17 & 16 & 0 & 5.806885 & -2.438681 & -0.331264 \\
\hline 18 & 6 & 0 & -1.197757 & 4.375203 & 0.471460 \\
\hline 19 & 6 & 0 & -0.691439 & 5.654951 & 0.358234 \\
\hline 20 & 6 & 0 & 0.717817 & 5.731376 & 0.191801 \\
\hline 21 & 6 & 0 & 1.364604 & 4.509572 & 0.170391 \\
\hline 22 & 16 & 0 & 0.160437 & 3.152395 & 0.368653 \\
\hline 23 & 6 & 0 & 2.714314 & 4.182054 & 0.014606 \\
\hline 24 & 6 & 0 & 3.861337 & 3.774748 & -0.127890 \\
\hline 25 & 6 & 0 & -4.793298 & 2.665210 & 0.969627 \\
\hline 26 & 6 & 0 & 5.110225 & 3.175544 & -0.316549 \\
\hline 27 & 16 & 0 & -6.001551 & 2.482241 & -0.403864 \\
\hline 28 & 6 & 0 & -6.994118 & 1.381085 & 0.634082 \\
\hline 29 & 6 & 0 & -6.441548 & 1.234187 & 1.875827 \\
\hline 30 & 6 & 0 & -5.219455 & 1.946330 & 2.063511 \\
\hline 31 & 6 & 0 & 6.386475 & 3.695525 & -0.351610 \\
\hline 32 & 6 & 0 & 7.414787 & 2.728710 & -0.578943 \\
\hline 33 & 6 & 0 & 6.962109 & 1.444339 & -0.720452 \\
\hline 34 & 16 & 0 & 5.160107 & 1.364015 & -0.581185 \\
\hline 35 & 6 & 0 & 8.043623 & -0.706519 & 0.248302 \\
\hline 36 & 6 & 0 & 7.721521 & 0.190526 & -0.981524 \\
\hline 37 & 6 & 0 & -8.244555 & 0.754782 & 0.115324 \\
\hline 38 & 6 & 0 & -8.162555 & -0.141543 & -1.159916 \\
\hline 39 & 6 & 0 & 2.429945 & -3.994949 & 0.800056 \\
\hline 40 & 6 & 0 & 3.564432 & -3.555922 & 0.946171 \\
\hline 41 & 6 & 0 & -3.593515 & 3.364184 & 0.792416 \\
\hline 42 & 6 & 0 & -2.507833 & 3.912001 & 0.640345 \\
\hline 43 & 8 & 0 & -8.735358 & -0.194321 & 1.125119 \\
\hline 44 & 8 & 0 & -9.363473 & -0.989649 & -0.983454 \\
\hline 45 & 8 & 0 & 9.066267 & 0.516180 & -1.464657 \\
\hline 46 & 8 & 0 & 9.160989 & -1.513659 & -0.290143 \\
\hline 47 & 6 & 0 & 9.917045 & -0.638271 & -1.173209 \\
\hline 48 & 6 & 0 & -9.622954 & -1.124336 & 0.444159 \\
\hline 49 & 1 & 0 & -1.823086 & -5.950312 & 1.132081 \\
\hline 50 & 1 & 0 & 0.712924 & -6.109112 & 1.731212 \\
\hline 51 & 1 & 0 & -6.504605 & -0.476414 & -3.436643 \\
\hline 52 & 1 & 0 & -4.350187 & -1.952173 & -3.306507 \\
\hline 53 & 1 & 0 & 4.955066 & -2.657244 & 3.252734 \\
\hline 54 & 1 & 0 & 7.133536 & -1.256635 & 2.890271 \\
\hline 55 & 1 & 0 & -1.332815 & 6.526646 & 0.395249 \\
\hline 56 & 1 & 0 & 1.254600 & 6.665709 & 0.086606 \\
\hline 57 & 1 & 0 & -6.898082 & 0.608071 & 2.629610 \\
\hline 58 & 1 & 0 & -4.645299 & 1.914220 & 2.980885 \\
\hline 59 & 1 & 0 & 6.580241 & 4.752930 & -0.221883 \\
\hline 60 & 1 & 0 & 8.464919 & 2.975745 & -0.661068 \\
\hline 61 & 1 & 0 & 8.414863 & -0.066610 & 1.060122 \\
\hline 62 & 1 & 0 & 7.205037 & -0.427248 & -1.729745 \\
\hline 63 & 1 & 0 & -9.021680 & 1.512087 & -0.069365 \\
\hline 64 & 1 & 0 & -8.352421 & 0.448013 & -2.060052 \\
\hline 65 & 1 & 0 & 10.824202 & -0.261145 & -0.692609 \\
\hline 66 & 1 & 0 & 10.144534 & -1.200816 & -2.081671 \\
\hline 67 & 1 & 0 & -9.382969 & -2.127361 & 0.802869 \\
\hline 68 & 1 & 0 & -10.672871 & -0.870555 & 0.616 \\
\hline
\end{tabular}


Compound: 13-i (See Figure S16)

Method: B3LYP/6-31G(d)

Key word: opt freq scf=(direct, tight)

Symmetry: $C_{1}$

\# of imaginary frequencies: 0

Energy: -4149.1146294 Hartree
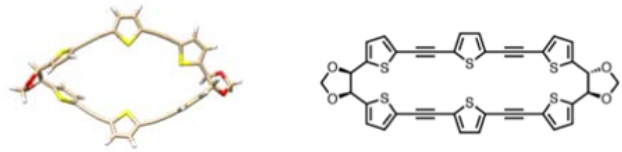

Table S23. Cartesian coordinate of optimized structure of 13-ii at B3LYP/6-31G(d) level.

\begin{tabular}{|c|c|c|c|c|c|}
\hline \multirow{2}{*}{$\begin{array}{l}\text { Center } \\
\text { Number }\end{array}$} & \multirow{2}{*}{$\begin{array}{l}\text { Atomic } \\
\text { Number }\end{array}$} & \multirow{2}{*}{$\begin{array}{l}\text { Atomic } \\
\text { Type }\end{array}$} & \multicolumn{3}{|c|}{ Coordinates (Angstroms) } \\
\hline & & & $\mathrm{X}$ & $\mathrm{Y}$ & $\mathrm{Z}$ \\
\hline 1 & 6 & 0 & -1.252388 & -4.115959 & -0.333019 \\
\hline 2 & 6 & 0 & -0.862694 & -5.351489 & 0.146678 \\
\hline 3 & 6 & 0 & 0.522284 & -5.465848 & 0.446347 \\
\hline 4 & 6 & 0 & 1.262813 & -4.325269 & 0.210044 \\
\hline 5 & 16 & 0 & 0.187390 & -2.998870 & -0.444109 \\
\hline 6 & 6 & 0 & -2.519993 & -3.623319 & -0.663103 \\
\hline 7 & 6 & 0 & -3.581414 & -3.071624 & -0.926544 \\
\hline 8 & 6 & 0 & -4.779636 & -2.391816 & -1.174302 \\
\hline 9 & 6 & 0 & 4.952055 & -2.963239 & 0.950467 \\
\hline 10 & 16 & 0 & -6.114367 & -2.373954 & 0.075532 \\
\hline 11 & 6 & 0 & -7.074898 & -1.271709 & -0.980080 \\
\hline 12 & 6 & 0 & -6.417911 & -1.009554 & -2.154492 \\
\hline 13 & 6 & 0 & -5.136634 & -1.625711 & -2.263924 \\
\hline 14 & 6 & 0 & 5.470644 & -2.574499 & 2.166775 \\
\hline 15 & 6 & 0 & 6.641042 & -1.766735 & 2.076846 \\
\hline 16 & 6 & 0 & 7.068302 & -1.514020 & 0.799094 \\
\hline 17 & 16 & 0 & 6.012186 & -2.352769 & -0.411332 \\
\hline 18 & 6 & 0 & -1.375702 & 4.294279 & 0.242104 \\
\hline 19 & 6 & 0 & -0.837811 & 5.562982 & 0.359465 \\
\hline 20 & 6 & 0 & 0.580057 & 5.613879 & 0.361681 \\
\hline 21 & 6 & 0 & 1.204428 & 4.385085 & 0.246910 \\
\hline 22 & 16 & 0 & -0.040119 & 3.052216 & 0.129969 \\
\hline 23 & 6 & 0 & 2.560758 & 4.056477 & 0.190011 \\
\hline 24 & 6 & 0 & 3.726980 & 3.688832 & 0.108101 \\
\hline 25 & 6 & 0 & -5.174694 & 3.000618 & -0.004593 \\
\hline 26 & 6 & 0 & 5.014471 & 3.163194 & -0.032712 \\
\hline 27 & 16 & 0 & -5.623656 & 1.391303 & 0.737893 \\
\hline 28 & 6 & 0 & -7.312111 & 1.595660 & 0.134599 \\
\hline 29 & 6 & 0 & -7.459799 & 2.787032 & -0.531164 \\
\hline 30 & 6 & 0 & -6.271965 & 3.568773 & -0.619181 \\
\hline 31 & 6 & 0 & 6.259368 & 3.743876 & 0.090146 \\
\hline 32 & 6 & 0 & 7.353792 & 2.856666 & -0.150231 \\
\hline 33 & 6 & 0 & 6.985039 & 1.574794 & -0.459238 \\
\hline 34 & 16 & 0 & 5.184397 & 1.395658 & -0.475731 \\
\hline 35 & 6 & 0 & 8.145719 & -0.582261 & 0.394546 \\
\hline 36 & 6 & 0 & 7.828740 & 0.390589 & -0.777518 \\
\hline 37 & 6 & 0 & -8.361427 & 0.572823 & 0.362368 \\
\hline 38 & 6 & 0 & -8.389030 & -0.733716 & -0.523471 \\
\hline 39 & 6 & 0 & 2.610792 & -4.032345 & 0.451039 \\
\hline 40 & 6 & 0 & 3.732102 & -3.593057 & 0.675956 \\
\hline 41 & 6 & 0 & -3.865642 & 3.484660 & 0.096292 \\
\hline 42 & 6 & 0 & -2.711268 & 3.886549 & 0.180532 \\
\hline 43 & 8 & 0 & -8.256053 & 0.014812 & 1.717489 \\
\hline 44 & 8 & 0 & -9.066758 & -1.697440 & 0.359411 \\
\hline 45 & 8 & 0 & 9.174635 & 0.823401 & -1.165155 \\
\hline 46 & 8 & 0 & 9.323232 & -1.290261 & -0.153344 \\
\hline 47 & 6 & 0 & 10.070199 & -0.309799 & -0.928178 \\
\hline 48 & 6 & 0 & -9.109759 & -1.145841 & 1.718288 \\
\hline 49 & 1 & 0 & -1.570738 & -6.158265 & 0.287943 \\
\hline 50 & 1 & 0 & 0.972884 & -6.368554 & 0.839491 \\
\hline
\end{tabular}




\begin{tabular}{lllrrr}
51 & 1 & 0 & -6.833825 & -0.373871 & -2.927672 \\
52 & 1 & 0 & -4.485723 & -1.508296 & -3.121073 \\
53 & 1 & 0 & 4.986786 & -2.826951 & 3.101546 \\
54 & 1 & 0 & 7.137829 & -1.347552 & 2.943946 \\
55 & 1 & 0 & -1.464403 & 6.442213 & 0.441318 \\
56 & 1 & 0 & 1.142538 & 6.535447 & 0.442350 \\
57 & 1 & 0 & -8.407149 & 3.104830 & -0.952042 \\
58 & 1 & 0 & -6.220217 & 4.527446 & -1.119319 \\
59 & 1 & 0 & 6.383406 & 4.789715 & 0.341719 \\
60 & 1 & 0 & 8.391742 & 3.160885 & -0.120989 \\
61 & 1 & 0 & 8.451138 & 0.010794 & 1.267191 \\
62 & 1 & 0 & 7.384132 & -0.193776 & -1.595215 \\
63 & 1 & 0 & -9.346423 & 1.051376 & 0.247152 \\
64 & 1 & 0 & -9.032013 & -0.560214 & -1.392224 \\
65 & 1 & 0 & 10.935837 & 0.069109 & -0.377575 \\
66 & 1 & 0 & 10.364078 & -0.784654 & -1.867162 \\
67 & 1 & 0 & -8.706003 & -1.868552 & 2.425637 \\
\hline.--------------------------------------------------------------------------
\end{tabular}

Compound: 13-ii (See Figure S16)

Method: B3LYP/6-31G(d)

Key word: opt freq scf=(direct, tight)

Symmetry: $C_{1}$

\# of imaginary frequencies: 0
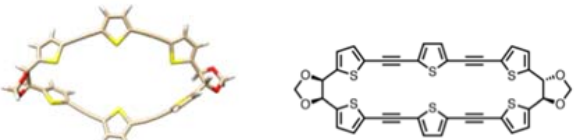

Energy: -4149.1173447 Hartree 


\section{S8. Reference}

1. Gaussian 16, Revision B.01, Frisch, M. J.; Trucks, G. W.; Schlegel, H. B.; Scuseria, G. E.; Robb, M. A.; Cheeseman, J. R.; Scalmani, G.; Barone, V.; Petersson, G. A.; Nakatsuji, H.; Li, X.; Caricato, M.; Marenich, A. V.; Bloino, J.; Janesko, B. G.; Gomperts, R.; Mennucci, B.; Hratchian, H. P.; Ortiz, J. V.; Izmaylov, A. F.; Sonnenberg, J. L.; Williams-Young, D.; Ding, F.; Lipparini, F.; Egidi, F.; Goings, J.; Peng, B.; Petrone, A.; Henderson, T.; Ranasinghe, D.; V. G. Zakrzewski, J. Gao, N. Rega, Zheng, G.; Liang, W.; Hada, M.; Ehara, M.; Toyota, K.; Fukuda, R.; Hasegawa, J.; Ishida, M.; Nakajima, T.; Honda, Y.; Kitao, O.; Nakai, H.; Vreven, T.; Throssell, K.; Montgomery, Jr., J. A.; Peralta, J. E.; Ogliaro, F.; Bearpark, M. J.; Heyd, J. J.; Brothers, E. N.; Kudin, K. N.; Staroverov, V. N.; Keith, T. A.; Kobayashi, R.; Normand, J.; Raghavachari, K.; Rendell, A. P.; Burant, J. C.; Iyengar, S. S.; Tomasi, J.; Cossi, M.; Millam, J. M.; Klene, M.; Adamo, C.; Cammi, R.; Ochterski, J. W.; Martin, R. L.; Morokuma, K.; Farkas, O.; Foresman, J. B.; Fox, D. J., Gaussian, Inc., Wallingford CT, 2016. 